\title{
NON-INVASIVE MODELING OF INTRACRANIAL HYPERTENSION FROM PHYSIOLOGICAL CHANNELS
}

by

\section{Parisa Naraei}

MSc, Computer Science (Information Security), University of Technology, Malaysia, 2013 BSc, Information Technology, Rahman Sufi Razi institute for higher education, Iran, 2011

\author{
A dissertation \\ presented to Ryerson University \\ in partial fulfillment of the \\ requirements for the degree of \\ Doctor of Philosophy \\ in the program of \\ Computer Science
}

Toronto, Ontario, Canada, 2019

(C) Parisa Naraei, 2019 


\section{AUTHOR'S DECLARATION FOR ELECTRONIC SUBMISSION OF DISSERTATION}

I hereby declare that I am the sole author of this dissertation. This is a true copy of the dissertation, including any required final revisions, as accepted by my examiners.

I authorize Ryerson University to lend this dissertation to other institutions or individuals for the purpose of scholarly research.

I further authorize Ryerson University to reproduce this dissertation by photocopying or by other means, in total or in part, at the request of other institutions or individuals for the purpose of scholarly research.

I understand that my dissertation may be made electronically available to the public. 


\title{
Abstract
}

\section{Non-invasive Modeling of Intracranial Hypertension from Physiological Channels}

\author{
Parisa Naraei \\ Doctor of Philosophy, 2019 \\ Computer Science \\ Ryerson University
}

\begin{abstract}
Intracranial pressure (ICP), the pressure within the cranium reflects three elements: cerebrospinal fluid, brain tissue and blood pressure. High ICP (above $20 \mathrm{mmHg}$ ) is called intracranial hypertension (ICH) which is due to the tumour, swelling or the internal bleeding of brain and may cause secondary damage to the brain. ICP is a crucial parameter in diagnosis of brain injuries. Two models which utilize machine learning techniques to anticipate ICH and assist in clinical decision making were developed in the present thesis.
\end{abstract}

ICP can be monitored through the invasive techniques (i.e., inserting an intraventricular catheter through the skull). Despite the high accuracy, the episodes of ICH can also be manually identified only after placement of catheter which is accompanied by lots of technical difficulties. Furthermore, the ICP signal might not be available continuously or may include unwanted noise that could introduce more complication to the diagnosis and treatment procedure.

Considering the difficulties of the invasive techniques, a non-invasive model, capable to predict the ICH helps to save time, estimate the missing ICPs, predict the ICP in advance and accelerate medical intervention. The present thesis introduces two machine learning models to resolve the current limitations: 1- Non-invasive prediction of ICP labels 10 minutes in advance where the status of ICP (normal / ICH) is predicted based on the two components extracted from the physiological signals such as mean arterial blood pressure and respiration rate. 2- Wavelet clustering where a machine learning solution for ICP estimation using a hybrid wavelet clustering is proposed. The episodes of ICP and derived from ICP (such as cerebral perfusion pressure) are excluded from the second model. 
The results indicated that a neural network gave the accuracy of $86.8 \%$ and the sensitivity of 94.4\% (AUROC=0.86), higher than the benchmark (AUROC=0.81). The clustering approach, which was tested via classification using the labels demonstrated a mean squared error of 0.19 mmHg. Clustering wavelet plots of correlated physiological signals with ICP and understanding changes of ICP from them, is experimented for the first time in this thesis. 


\section{Acknowledgements}

Embarking on a Ph.D. degree is not an easy endeavor and I have been lucky enough to have had the support of the best colleagues, friends, and family one could wish for. First and foremost, I wish to thank my advisor, Professor Dr. Alireza Sadeghian, director of the Initiative Intelligence Laboratory for his invaluable support and encouragement through these years. He gave me the moral support and wise consultation on the topic. Moreover, I want to express my gratitude to Dr. Michael Cusimano, Neurosurgeon and Scientist at St.Michael's hospital for giving me the opportunity to work on a topic that I found very interesting both from the machine learning and medical perspective. Also to Dr. Mohsen Nouri, neurosurgeon and endovascular surgical neuroradiology from Icahn school of medicine at Mount Sinai for his invaluable support and discussing all of the medical aspects of this project with me and taking me through each and every phase of managing traumatic brain injured patients and the surgery decision making process. Moreover, I would like to appreciate my gratitude to the members of my committee Professor Dr. Nahavandi, Professor Dr.Geurkov, Professor Dr. Ding and Professor Dr. Harley for guiding me through the path.

I acknowledge the Ontario Trillium scholarship I was awarded throughout my Ph.D. research; the financial assistance from my supervisor through the Brain Alliance big data research, analytics and information network ORF-RE and the graduate development award from the Ryerson School of Graduate Studies. 


\section{Dedication}

My Ph.D. thesis is dedicated to my parents and my brother for enabling me to chase my dreams. For always motivating me and believing in me. They are the most precious gifts in my life. I cannot express how grateful I am to have them as the lights in the darkest nights.

It is dedicated to my husband Dr.Hadavi for his love and support who is my best friend and my wise consultant.

It is dedicated to my grandparents who always wanted to see me go anywhere as long as it is forward in education.

Finally, it is dedicated to all of the strong women in the world who never stop believing in themselves and empowering others. 


\section{Table of Contents}

AUTHOR'S DECLARATION FOR ELECTRONIC SUBMISSION OF DISSERTATION ........ ii

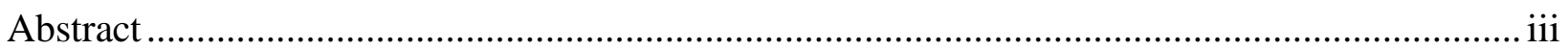

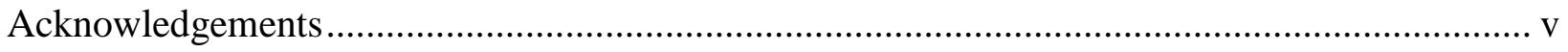

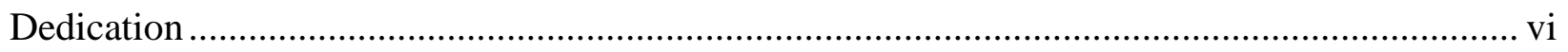

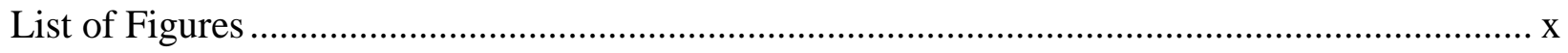

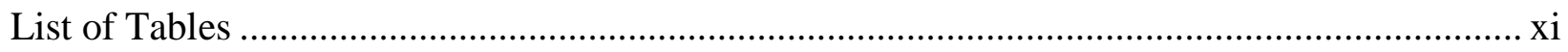

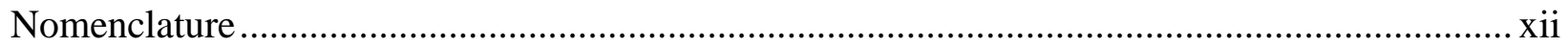

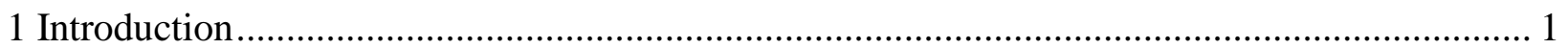

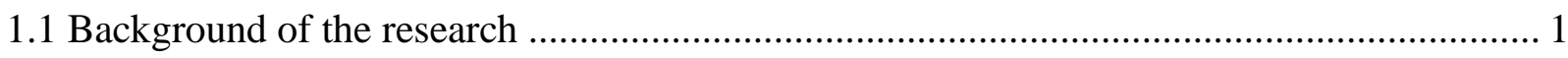

1.2 Problem statement with ICP collection.................................................................... 1

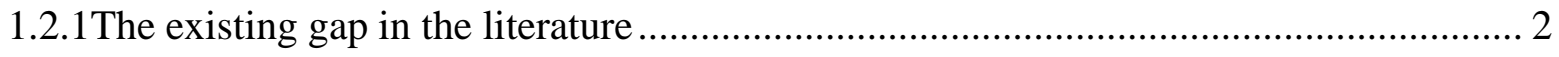

1.3 Non-invasive predicting of Intracranial Hypertension .......................................... 2

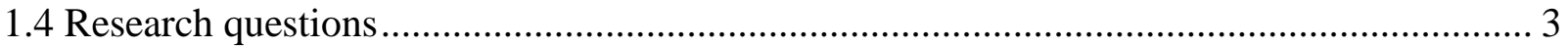

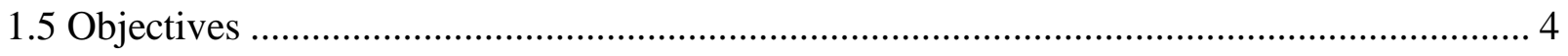

2 A review of Intracranial Pressure Signal Analysis and Machine Learning Inspired Techniques 5

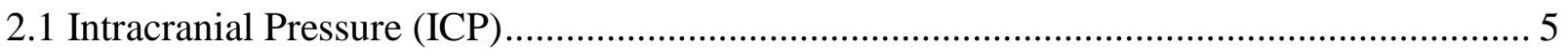

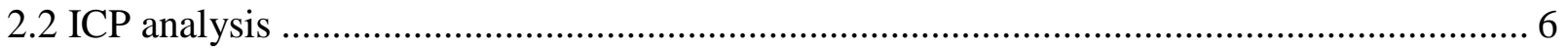

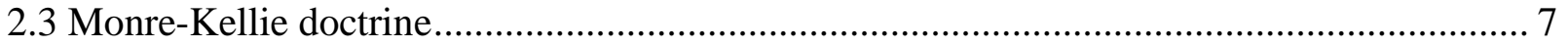

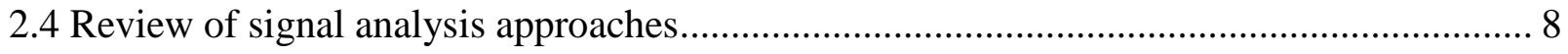

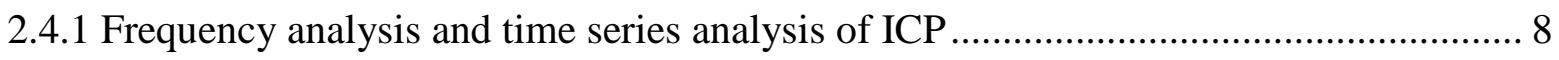

2.4.2 Brain compliance and pressure reactivity index................................................. 9

2.4.3 Entropy-based ICP trend analysis ............................................................... 10

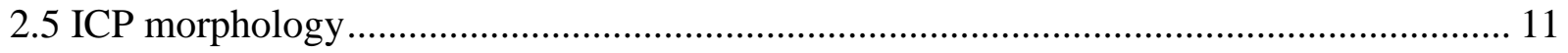

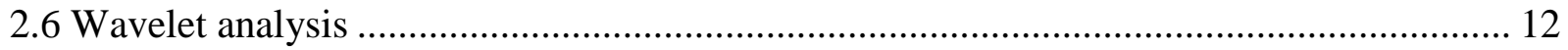

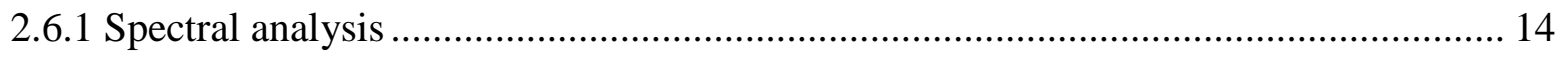

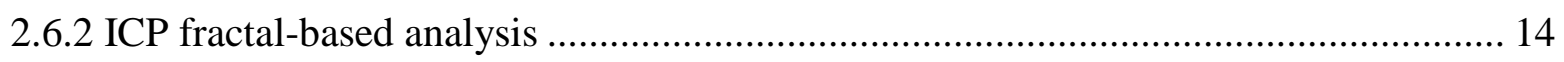

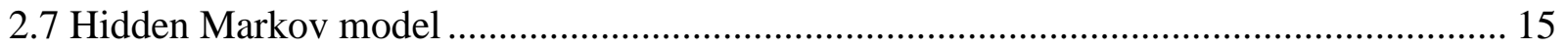




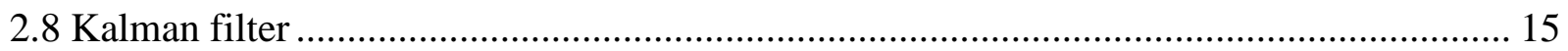

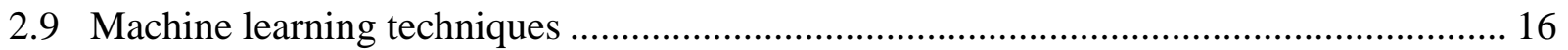

2.9.1 Logistic regression and artificial neural networks .................................................. 16

2.9.2 Support vector machines ........................................................................................ 18

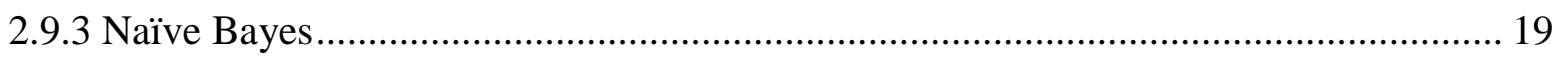

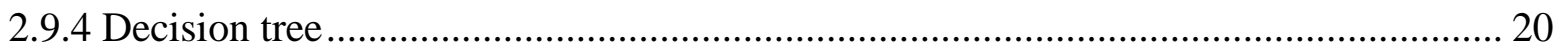

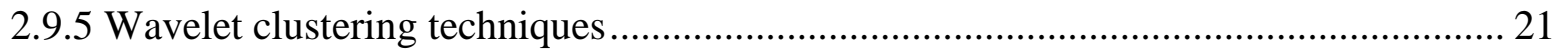

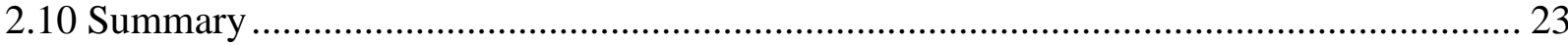

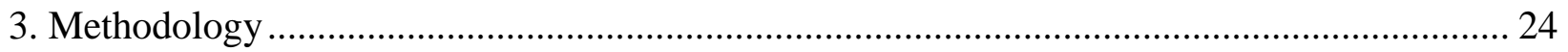

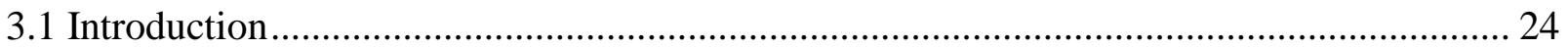

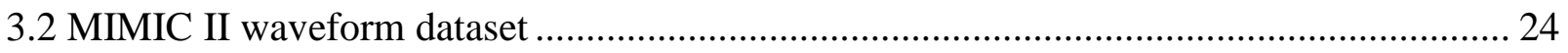

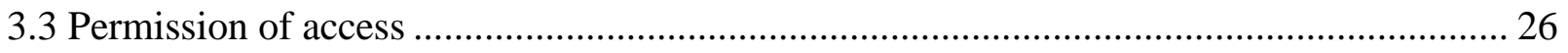

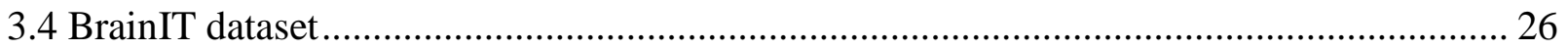

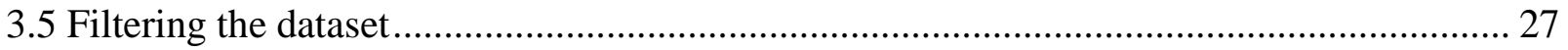

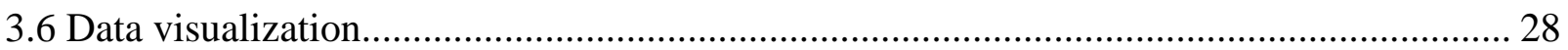

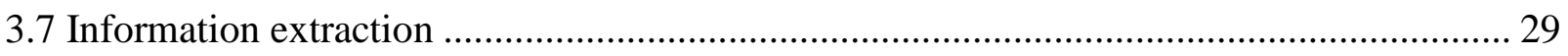

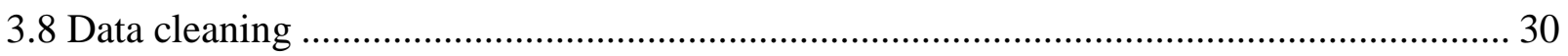

3.9 Finding correlation: Pearson correlation coefficient.......................................................... 32

3.10 Feature selection: Pearson correlation, information gain and PCA ................................... 32

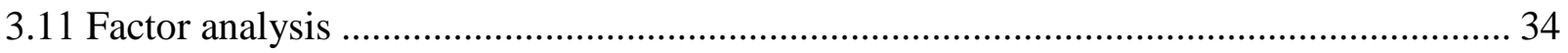

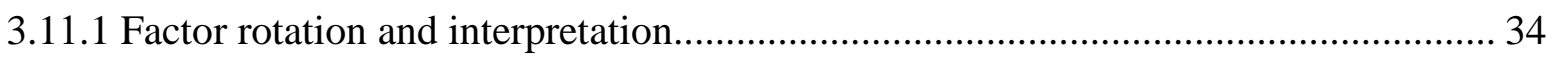

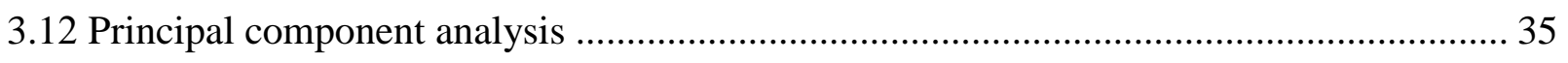

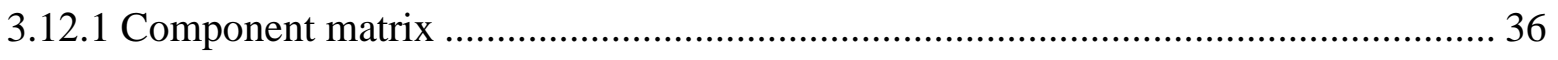

3.13 Proposed solution: supervised methodology ………………………………………...... 36

3.14 Proposed solution: unsupervised methodology …………………………………...... 40

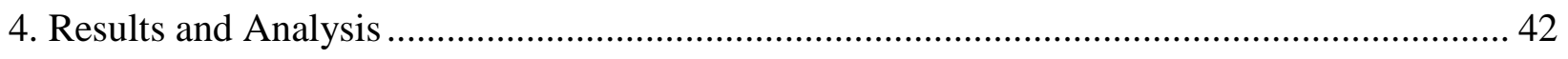

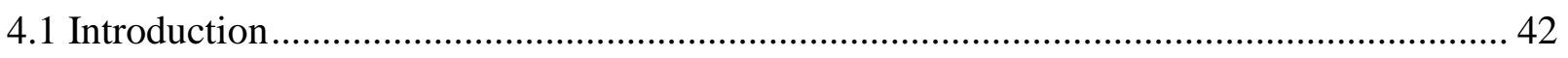

4.2 Result of Pearson correlation analysis and information gain.............................................. 43

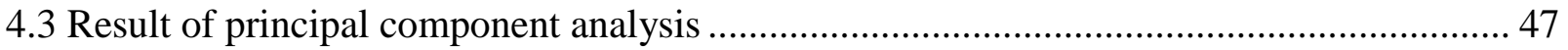

4.4 Result of Prediction of Normal ICP vs. Intracranial Hypertension ..................................... 56

4.5 Result of Clustering Physiological Signals ......................................................................... 62

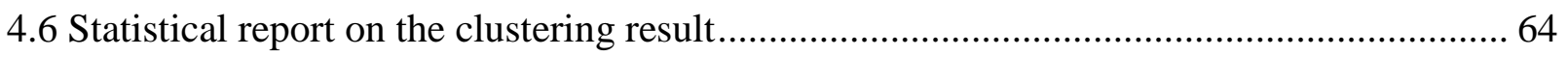


4.7 Evaluation of Clustering Labels with Classification ................................................. 66

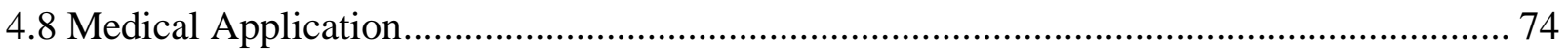

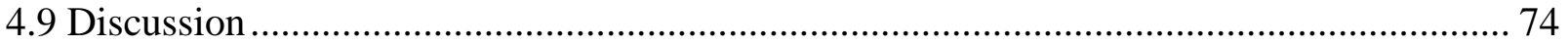

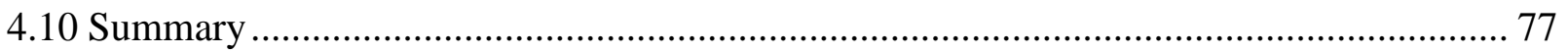

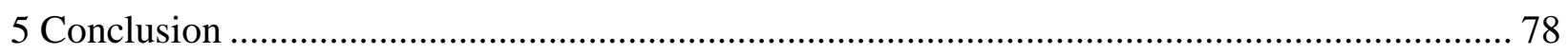

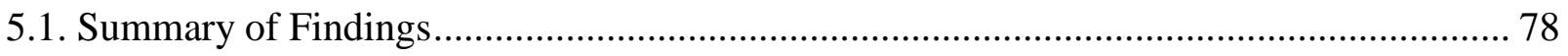

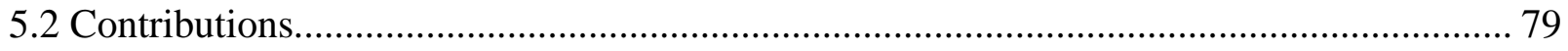

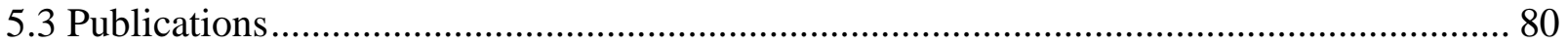

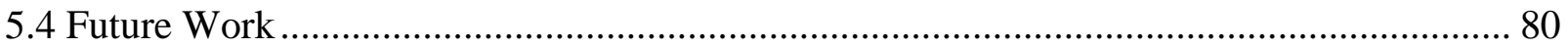

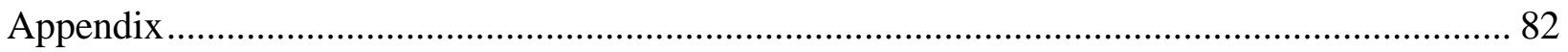

Appendix A. Wavelet Plots: samples of pre-raised ICH ............................................... 82

Appendix B. Extraction of the raised episodes of ICP ................................................... 84

Appendix C. Implementation of the predictive model............................................... 87

Appendix D. Sample result of PCA (Fact 1 and Fact 2 as Inputs to Neural Network) ............ 88

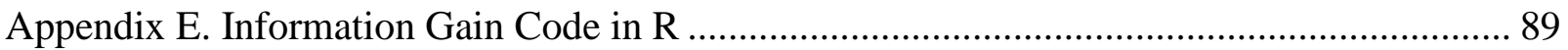

Appendix F. Selecting patients with normal lead before episodes of highICP (Checking for 5,

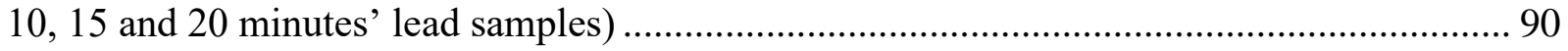

Appendix G. K-means Clustering on Wavelet plots.......................................................... 91

Appendix H. Classification of K-means Result .................................................................. 95

Appendix I. Creating Wavelet-plots ........................................................................... 104

Appendix J. Performance of the best trained model in MIMIC II Waveform database ( 2 hidden

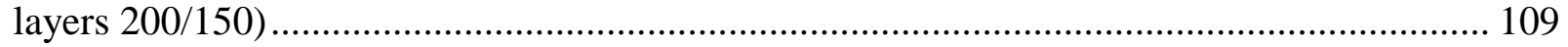

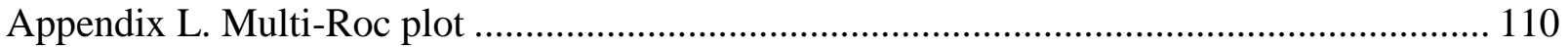

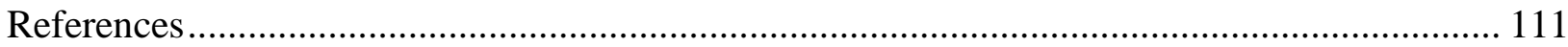




\section{List of Figures}

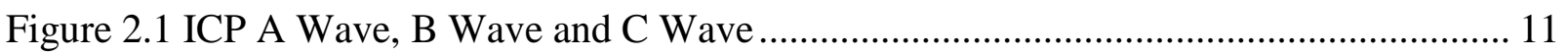

Figure 2.2 a sample of ICP signal of a traumatic brain injured patient over an episode of 1200

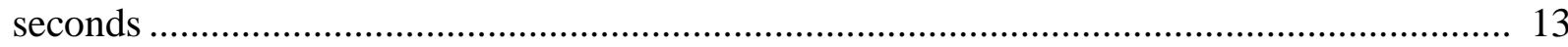

Figure 2.3 wavelet power levels corresponding to the same patient in Fig. 2.2 …………....... 13

Figure 3.1 Signal availability statistics for the MIMIC II data samples ................................. 26

Figure 3.2 Scatterplot of Intracranial pressure vs. blood pressure........................................... 28

Figure 3.3 Parallel coordinates of correlating body signals with ICP ..................................... 27

Figure 3.4 Original ICP magnitude plot …………….................................................. 31

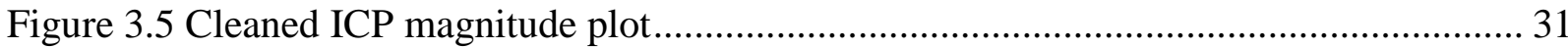

Figure 3.6 Evaluating different time intervals from "pre-raised episodes" to "intracranial hypertension episodes" ............................................................................................ 31

Figure 3.7 Non-invasive predictive methodology diagram...................................... 38

Figure 3.8 Architecture of the feed forward neural network ............................................ 31

Figure 3.9 Clustering methodology diagram ...................................................................... 41

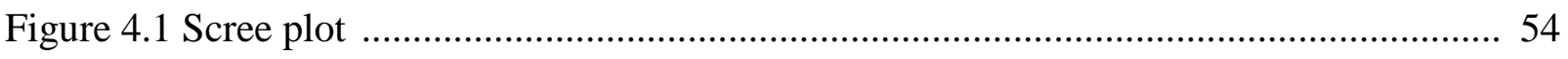

Figure 4.2 Predictive model performance of specificity, accuracy, precision and Sensitivity .. 58

Figure 4.3 False Positive Rate vs. True Positive Rate (model 300-150) .................................... 60

Figure 4.4 False Positive Rate vs. True Positive Rate (model 400-400) …………….............. 60

Figure 4.5 False Positive Rate vs. True Positive Rate (model 100-150) ………………............. 61

Figure 4.6 False Positive Rate vs. True Positive Rate (model 200-150) ................................... 61

Figure 4.7 False Positive Rate vs. True Positive Rate (model 300-300) .................................. 62

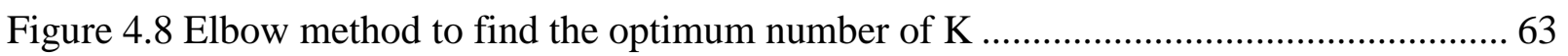

Figure 4.9 the accuracy of classification of the clustering result .............................................. 66

Figure 4.10 Sensitivity of classification of the clustering result .............................................. 67

Figure 4.11 Precision of classification of the clustering result ................................................. 68

Figure 4.12 Specificity of classification of the clustering result ............................................ 64

Figure 4.13 Mean squared error of classification of the clustering result ................................ 70

Figure 4.14 ROC of different classes obtained by multinomial logistic regression ................. 72

Figure 4.15 ROC of different classes obtained by perceptron classifier .................................. 73 


\section{List of Tables}

Table 4.1 The result of Pearson's correlation ................................................................... 44

Table 4.2 Information gain analysis and attribute selection with respect to class labels of ICP45

Table 4.3Information gain analysis from clinical attributes with respect to class labels of ICP46

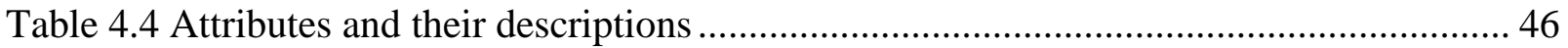

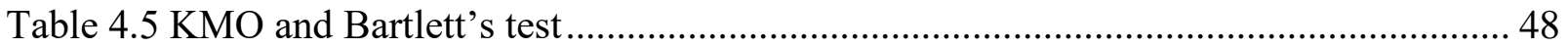

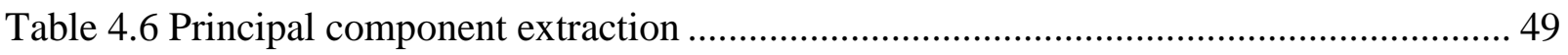

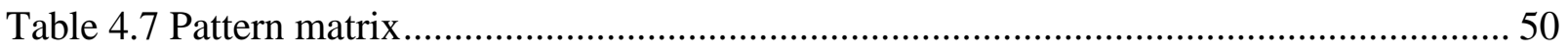

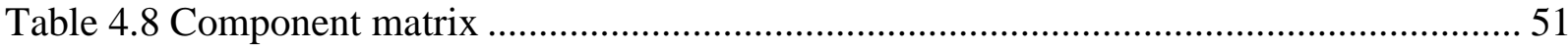

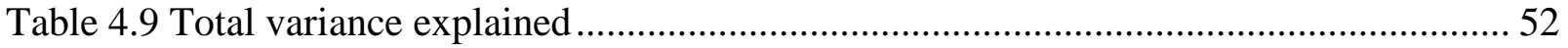

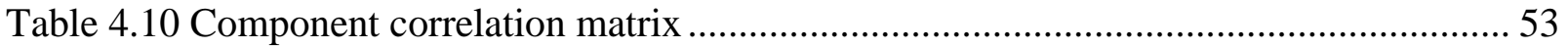

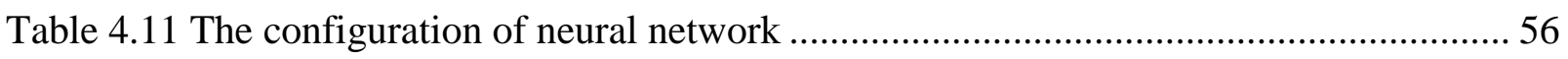

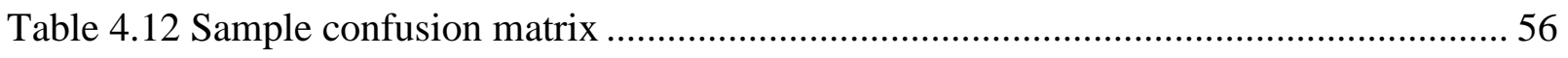

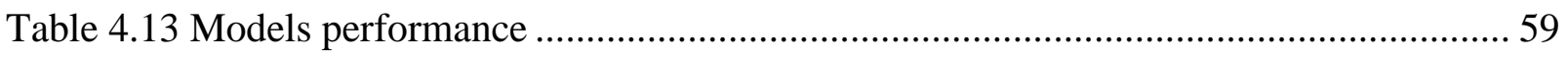

Table 4.14 Patients' condition corresponding to cluster 0(Gained normal label: 48 members) 65

Table 4.15 Patients' condition corresponding to cluster 1 (Gained the label of pre-raised ICP:

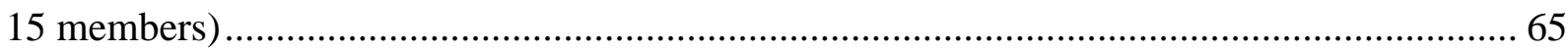

Table 4.16 Patients' condition corresponding to cluster 2 (gained the label of intracranial

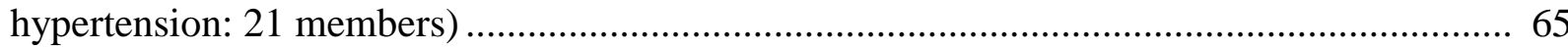

Table 4.17 the evaluation of the implemented classifiers on the labels................................ 71

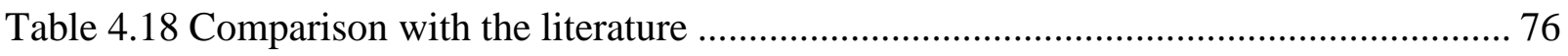




\section{Nomenclature}

\begin{tabular}{|c|c|}
\hline$I C P$ & Intracranial Pressure \\
\hline$I C U$ & Intensive Care Unit \\
\hline$H Z$ & Hertz \\
\hline$A C F$ & Auto-correlation Function \\
\hline$D F A$ & Detrended Fluctuation Analysis \\
\hline$R M S$ & Root Mean Squared \\
\hline$A B P$ & Arterial Blood Pressure \\
\hline $\operatorname{Resp}$ & Uncalibrated Respiration Waveform \\
\hline$H R$ & Heart rate \\
\hline$P V C$ & Premature Ventricular Contraction \\
\hline$C P P$ & Cerebral Perfusion Pressure \\
\hline ST III, ST V & ST Segments in Electrocardiography \\
\hline$A N N$ & Artificial Neural Networks \\
\hline$P C A$ & Principal Component Analysis \\
\hline$S V M$ & Support Vector Machine \\
\hline ABP Means & Mean Arterial Blood Pressure \\
\hline Pulse & The Difference Between Systolic and Diastolic Blood Pressure \\
\hline ABP Systolic & Maximum Arterial Blood Pressure \\
\hline ABP Diastolic & Minimum Arterial Blood Pressure \\
\hline Pleth & Uncalibrated Row Output of Fingerprint Plethysmograph \\
\hline SPO2 & Peripheral Capillary Oxygen Saturation \\
\hline KMO & Kasire Meyer Olkin Test \\
\hline$F F T$ & Fast Fourier Transform \\
\hline$T C$ & Lung Total Capacity \\
\hline $\mathrm{SaO} 2$ & Amount of oxygen bound to hemoglobin in arterial blood \\
\hline GCS Eye & Coma severity based on Eye \\
\hline
\end{tabular}


GCS Verbal

$\mathrm{FiO} 2$
Coma severity based on verbal response

Fraction of Inspired Oxygen 


\section{Introduction}

\subsection{Background of the research}

Traumatic brain injury (TBI) is the main reason for the secondary human brain injury and death on the world. Although, the primary injury in (TBI) patients is diagnosed using brain imaging, the secondary brain injury, which may also lead to the following long term and/or permanent brain impairment remain as the main risk [1] such as: cerebral hypoxia defined as low brain oxygen flow, brain herniation described as brain swelling that may result in brain compression in skull and cerebral ischemia defined as low blood level in brain [2].

Any of these phenomena may aggravate the TBI patient condition and result in death [3]. The key parameter in understanding and prediction of the above problems and the secondary brain injury is ICP [4][5]. ICP is described as the pressure inside the intracranial cavity (skull) and is determined using the overall volume of brain tissue, blood and cerebrospinal fluid. ICP screening is a common practice in ICU units to monitor comatose TBI patients [6].

\subsection{Problem statement with ICP collection}

The existing invasive technique is based on the insertion of a transducer or catheter into the skull and insertion of intra-parenchymal ICP monitor. This practice requires the insertion of the catheter into the patient's brain [7]. Intra- or extra-cerebral hemorrhage usually terminates once the catheter is placed but skin stitches and bone wax might be possible if bleeding continues [8]. Although intracranial hemorrhage is rare, unenhanced CT scan of the head may be performed after the procedure if hemorrhage would be suspected. Infection is an uncommon but serious late complication which may adversely affect the outcome [8].

Prediction of intracranial hypertension (ICH) and patient outcome (survival, vegetarian life, death) based on the current health status and clinical recordings is crucially important for physicians [2]. Considering the important negative side effect and complexities associated with the invasive ICP monitoring various research groups have been looking into non-invasive approached [5][9]. Although the non-invasive techniques are not as accurate. Despite all the 
progress and valuable findings in non-invasive techniques, neither of them has been broadly used in clinical practices [2][9].

\subsubsection{The existing gap in the literature}

The limitation on the available models in the state of the art literature is that the proposed solutions are mainly targeting the problem of ICP monitoring being reactive and as a result, predictive models are proposed. The general solutions proposed are including ICP history as well as different other clinical variables as the inputs of the models and predicting future ICP accordingly. The predictive models of ICP are highly dependent on the ICP signal and even though a number of body signals are included in the feature space (such as arterial blood pressure, oxygen saturation, etc.) to predict ICP future values, the cerebral signals (ICP and CPP) are included as well as the independent variables for modeling. The contribution of all of such models would be to help in "automation" of ICP analysis procedure with the current medical settings.

What is yet to be discovered is whether a mapping could be defined between the routinely collected vital signals collected from the patients from the heart and respiration channels to cerebral channel showing the normal/intracranial hypertension behavior of ICP. On top of that a more fundamental question is yet to be answered which is whether a "structure" could be found in body signals to show patterns of changes of ICP. To achieve this goal, some fundamental questions are defined as the research questions in this thesis as disclosed in Sec 1.4.

\subsection{Non-invasive predicting of Intracranial Hypertension}

Monitoring of ICP in the current practice requires the intraventricular catheter to be inserted into the patient skull. It is direct and accurate but there are negative side effects. Insertion of the intraventricular catheter bolt has the risk of haemorrhage and the possibility of infection [4]. It is very desirable to have a sensitive and accurate predictive model to help anticipating the future ICP status, independent of ICP as the input. 
The prediction systems are expected to predict the forthcoming rise in the ICP; however, it would be very desirable if the prediction could also be conducted non-invasively and help in

managing and controlling the Traumatic Brain Injured (TBI). If available, the non-invasive predictive model of ICP, independent of the cerebral signals as the inputs, capable of forecasting normal versus intracranial hypertension states of the brain, few minutes in advance, could be an ideal decision support model to be integrated into the current medical setting.

The present study showed that some of the different body channels such as distinct leads of heart and respiratory system (i.e., systolic and diastolic blood pressure) are correlated with the status of ICP and the signals are vital signals easy to collect and available from the patients. The present thesis shows that the correlations are not only through body organs such as coronary heart and respiratory system but also through different leads of heart and breathing systems.

In this thesis, two non-invasive models are proposed. The first modelling is conducted using the components extracted from the most correlated body signals with ICP, in which ICP class label (normal/raised) is predicted. The second proposed model based on unsupervised learning investigates the possible structure in the correlating body signals with ICP and accordingly 3 clusters are defined and labelled. In this thesis, the assumption to be hold for modeling would be having access to a window of biomedical signals as well as ICP, where the fluctuation of signal is showing both normal and raised episodes of ICP. In this thesis, the following research questions are investigated.

\subsection{Research questions}

The research questions that require to be answered would be as follows:

- $\quad$ Are there other body channels correlating with the status of ICP?

- $\quad$ Are these correlations significant?

- $\quad$ Are the correlated body signals able to show some patterns of changes of ICP?

- Is it possible to predict the future ICP value from correlating physiological signals?

- Is it possible to build a predictive model to have the clinical application? 
- Is it possible to define a structure from correlating physiological signals with ICP and interpret those to understand possible status of ICP (normal, pre-raisedraised)?

\subsection{Objectives}

- To investigate the feasibility of modeling ICP from physiological channels: investigating the correlation between physiological features and Intracranial Pressure.

- $\quad$ To investigate predictively of ICP from various channels and leads of heart such as Central Venous Pressure, Heart Rate, Pulse, Diastolic Arterial Blood Pressure, Respiration and Mean Arterial Blood Pressure.

- $\quad$ To solve the multicollinearity problem detected in physiological data.

- $\quad$ To conduct predictive modeling of ICP from correlated physiological signals with ICP.

- $\quad$ To conduct clustering and finding structures from correlated physiological signals with ICP. 


\section{A review of Intracranial Pressure Signal Analysis and Machine Learning Inspired Techniques}

Various signal analysis techniques utilized to assess ICP and machine learning inspired approaches for ICP analysis are reviewed in this chapter. Generally, most of the studies in this field engage the time series modeling and forecasting; and the main models introduced in the present thesis work based on signal analysis and machine learning approaches.

\subsection{Intracranial Pressure (ICP)}

Intracranial pressure (ICP), also known as the internal skull pressure is a crucially important parameter that can influence the functioning and vital symptoms of brain. Several internal elements such as the brain tissue, cerebrospinal fluid (CSF) and blood pressure, as well as external factors such as sneezing, coughing, and movements can alter the ICP [10]. Alteration of ICP is often due to volume changes in one or more than one of the organs in the skull. ICP can be quantified in the unit of millimeters of mercury ( $\mathrm{mmHg}$ ), however there is some controversy about the normal and elevated ICP range among the clinicians. While many clinicians perceive ICP values in the range of $0-15 \mathrm{mmHg}$ as regular [11], [10], [12], [13], ICP magnitudes in the range of 20-25 $\mathrm{mmHg}$ is considered as elevated and patient need to receive therapeutic intervention [14]. Variations of ICP also depends on age. For instance, the normal range of ICP in infants and children is 1.5 to $6.0 \mathrm{mmHg}$ and 3 to $7 \mathrm{mmHg}$, respectively [11], [15].

The critical ICP also known as intracranial hypertension is considered as the continually elevated ICP, usually over $20 \mathrm{mmHg}$ [16]. Intracranial hypertension may lead to the secondary injuries and can result in death; thus needs to be prevented through medical intervention such as cerebrospinal fluid drain [2] [17].

Time is a crucial parameter in clinical intervention of TBI patients. Increased ICP is generally observed in wide range of patients suffering from brain related symptoms such as hydrocephalus, intracranial tumors, cerebral edema and etc. [8]. Sudden rise of ICP is a critical parameter that needs timely detection and intervention; i.e., it can result in a wide range of 
problematic issues such as lowering cerebral perfusion pressure (CPP) or herniation of the brainstem which leads to serious neurological damages or death [3]. Stiver et al. [18] studied the variation of the severity of intracranial hypertension and the negative outcome of the intense head damage. Patients' response was observed to be critical while patient experiences elevated ICP and regular in patients with regular ICP [19]. This implies that due to elevated ICP, the TBI patients need to be monitored and receive medical intervention to reduce and control the ICP. Considering all these aspects, monitoring and recording ICP is an important element in assessing and medical intervention of TBI patients [20].

There are various techniques of ICP monitoring such as invasive or direct ICP monitoring techniques including subdural, ventricular cannulation, epidural, and intra-parenchymal devices and non-invasive (or indirect) including optic nerve sheath diameter, lumbar puncture, visual evoked potentials, ultrasound and Doppler [21], [22], [23], [24], [25], [26], [27] and [28]. The ICP shown on bedside monitors is collected through the invasive ICP measurement method; i.e., a pressure transducer penetrated into the subdural, epidural, intra-parenchymal, or lateral ventricular space [10].

\subsection{ICP analysis}

Traumatic brain injured (TBI) patients' outcome depends on various factors such as ICP, as one of the most important ones and respiration, cardiac conditions, body temperature, oxygenation and blood pressure [14]. Considering the importance of these parameters in determining and predicting the patient response, comprehensive pathophysiology and clinical decision making for TBI patients, these elements and their correlation with ICP have traditionally been researched using waveform analysis [29] and [30]. Moreover, ICP waves have been analyzed to explore further prognostic and diagnostic information [10].

Variations of biological signals was studied through nonlinear analysis and could be a helpful and reliable technique to explain ICP time series [30],[31],[32],[33]. Kim et al. [34] focused on noninvasive ICP (NICP) modeling and figured that Kernel spectral regression-based technique as a nonlinear mapping function enhances the efficacy of data mining framework for 
NICP compared to linear techniques. The advantage of nonlinear ICP models is to achieve high curve-fitting functionality.

The predicting models developed by Narotam et al. utilized regression analysis to correlate the oxygen level in brain tissue to other parameters such as brain temperature, CPP, ICP and pulmonary oxygenation [35]. The health conditions can be correlated through cohort analysis which demonstrates that the estimator of cerebral perfusion pressure $(\mathrm{eCPP})$ is correlated with CPP $(\mathrm{R}=0.851, \mathrm{p}<0.001) \pm$ mean standard deviation of $4.02 \pm 6.01 \mathrm{~mm} \mathrm{Hg}$, with less than $10 \mathrm{mmHg}$ error in $83.3 \%$ of occasions [36]. It was figured that eCPP estimates low CPP $(<70 \mathrm{~mm} \mathrm{Hg})$ with area under the curve (AUROC) equal to 0.913 [36].

The linear modeling could also be applied to explain the correlation among ICP and other parameters such arterial blood pressure, cerebral blood flow velocity, brain temperature and etc. [37] and [34].

\subsection{Monre-Kellie doctrine}

Monro-Kellie doctrine hypothesized that the total brain volume, consisted of cerebrospinal fluid (CSF) and intracranial blood is constant [38]; meaning that an increase in one results in decrease in another. Alexander Monro studied the cranial cavity from physics point of view and discovered that the volume of the blood circulating in cranium is hypothetically constant [39].

This doctrine explains the reason for many of the symptoms observed in TBI patients. For example, the elevated ICP in patients suffering from brain injury is due to the occupied volume by bleeding that results in modified CSF and/or blood. On the contrary, a decrease in brain volume will be filled by CSF and/or blood that can maintain the ICP [40]. 


\subsection{Review of signal analysis approaches}

ICP is considered as a useful indicator of intracranial condition; thus, the following critical literature review focused on waveform analysis will give helpful insight about the previous studies and important considerations.

\subsubsection{Frequency analysis and time series analysis of ICP}

The ICP value demonstrated on bedside monitors is the average of ICP signals collected in short time spans. The standard deviation can also be calculated for similar time [41]. O'Phelan et al. investigated the ICP collected from several TBI patients and figured that ICP waves follow distinct patterns [42]. Bellotti et al. and Eide et al. studied the variations of elevated ICP in TBI patients before and after surgery [43],[44]. It was emphasized that decrease of mean ICP is the key element in clinical interventions of patients suffering from intracranial hypertension [45]. However, it was later discovered that the mean ICP is not an accurate indicator of dynamic characteristics of ICP [44]. Thus, the emphasis was shifted to detecting the pulses in ICP waveform for computing the mean wave value; which is a more reliable and precise method to indicate intracranial compliance [44], [46], [47],[10].

Fast Fourier Transform (FFT) is a method to represent signals in frequency domain which was broadly used in 80's for extracting important parameters and better understanding of signals [48]. ICP signal is unpredictable to some extent and does not follow a complete sinusoid pattern. On the other side, using FFT method, it was assumed that the signal is stationary as there is only one peak in each cycle [48]. Due to the data reduction happening in the frequency-domain method by converting the signal from its time domain to frequency domain; FFT analysis is not appropriate method for ICP analysis as some important information of ICP signal revealing in time domain will be eliminated.

Klingelhöfer et al. [49] studied 13 patients with almost equivalent medical condition for whom the other parameters that influence the transcranial Doppler results was also considered to be constant $(\mathrm{r}=0.873 ; \mathrm{P}<0.001)$. They discovered that ICP and the magnitude of (mean systemic 
arterial pressure * Pourcelot index over the mean flow velocity) were found to be closely correlated [49],[10].

Considering the shortcoming of frequency domain methods, the time domain is highly preferred for computation of pulse amplitudes [48]. Holm et al. assessed the harmonics frequency of ICP as well as fundamental frequencies to better understand the ICP signal [48]. In order to assess the volume of information obtained from the early waveform of the primary harmonics, Holm et al. showed the results on the maximum point of the original ICP [48],[10].

\subsubsection{Brain compliance and pressure reactivity index}

Subordinate indices were found to be helpful in identify the threshold and commencement of intracranial disorder in 70 s along with waveform analysis. In order to estimate the intracranial instant capacity, the joint point between ICP pulse amplitude and the linear slopes of baseline ICP [50] and plots of standard deviation regression and mean ICP slope variations [51] were considered to indicate the variations of intracranial dynamics. The mean wave amplitudes and mean values of ICP were used as secondary indices [10].

Pressure reactivity index (PRx) was known to be required in efficient ICP analysis which also indicates the auto regulatory performance of the brain and cerebral perfusion pressure [10]. Barth et al [52], discovered the correlation between PRx, ABP waveform amplitudes and ICP pulse pressure. PRx results in a weak correlation with patient outcome in subarachnoid hemorrhage patients while associated with flow-related indices and oxygen. The correlation between ABP wave amplitude and patient outcome (survivors and non survivors as well as short-term and longterm) was utilized to address the PRx-ICP limitations [10].

Bajla et al. [53] proposed using a sliding window technique to demonstrate retrospective ICP data in real time. The pressure-volume curve is equivalent to the Monro-Kellie rule explained in section 2.3 and was characterized with the amplitude regression and pressure (RAP) coefficient [54] known as a correlation factor between pulse waveform amplitude and ICP [44]. The reactivity of cerebrovascular pressure indicated the ability of muscle tone in arterioles walls and cerebral arteries to respond to variations in transmural pressure [55]; which is considered as a measure of neurological compensation [56],[10]. 
Czosnyka and Pickard introduced the pressure reactivity index (PRx) as a representation of mean ICP and correlation coefficient of arterial blood pressure (ABP) [57], which was found to a be a powerful tool in anticipating poor outcome in TBI patients and was investigated by other scholars as well [57], [55], [56]. Human brain is capable of preserving a fairly constant blood flow while experiencing alteration in perfusion pressure, which is known as autoregulation of cerebral blood flow [58][10].

\subsubsection{Entropy-based ICP trend analysis}

Trend analysis is a useful technique applicable when there is a relation between variables, established based on the historical data. The shortcoming of trend analysis is that the historical data may not portray the realistic trend of the underlying trend perfectly; however, it can be replicated, updated, refined and checked, if needed [59]. The key challenge of this method is to detect the turning points, as it is difficult to identify them in real-time data, since they appear as the start point of new trends or deviation from the present trend. The accuracy of trend analysis for ICP relies on the historical ICP data available for the analysis which is often not present [2].

Degree of irregularity/complexity in such methods, which can be utilized to evaluate the variability of biomedical signals, depends on the order of the elements in the time series [30]. Approximate Entropy (ApEn) is a technique to evaluate pattern generations and signal randomness and measure the regularity in any biomedical time-series data [60]. ApEn can also evaluate the time series to identify patterns and frequency of their occurrence [41]. One of the important advantages in using ApEn relies in its ability to look into the underlying patterns commonly ignored by other methodologies (i.e., statistical time-domain or spectral frequency analysis) [61],[10].

The Lemple-Ziv compression entropy, a nonparametric measure, was utilized for one dimensional signals to evaluate the signal complexity and frequency of occurrence in the given time interval [62], [63]. ApEn along with the Lempel-Ziv were used to evaluate the ICP signal variations in TBI patients and determine the relations with mean ICP in Lempel-Ziv compression entropy measure [64], [61][10]. Some other biomedical signals (i.e., arterial blood pressure and 
heart rate) were also broadly utilized in entropy measure applications. Noticeable amount of research is still going on to predict and evaluate the complexity of ICP time series [62].

\subsection{ICP morphology}

Lundberg studied the ICP waveforms and classified the ICP into three wave patterns as shown in Fig. 2.1. Lundberg labeled the ICP waveform observed in intracranial hypertension, i.e., an unexpected elevated ICP beyond $50 \mathrm{mmHg}$ in 5 to 20 minutes, as wave type A [65]. Wave type $\mathrm{A}$ is an indicator of weak brain compliance [66]; which was also observed while a patient experiences low cerebral blood flow and cerebral perfusion pressure [45]. Low-frequency waves corresponding to ICP range of 10 to $20 \mathrm{mmHg}$ accompanied with alteration in breathing is labeled as "Pressure pulse B waves" and ultimately, Lundberg called the ICP waves associated with respiratory and cardiac signals having 4 to 8 oscillations per minute as "Traube-Hering $\mathrm{C}$ waves".

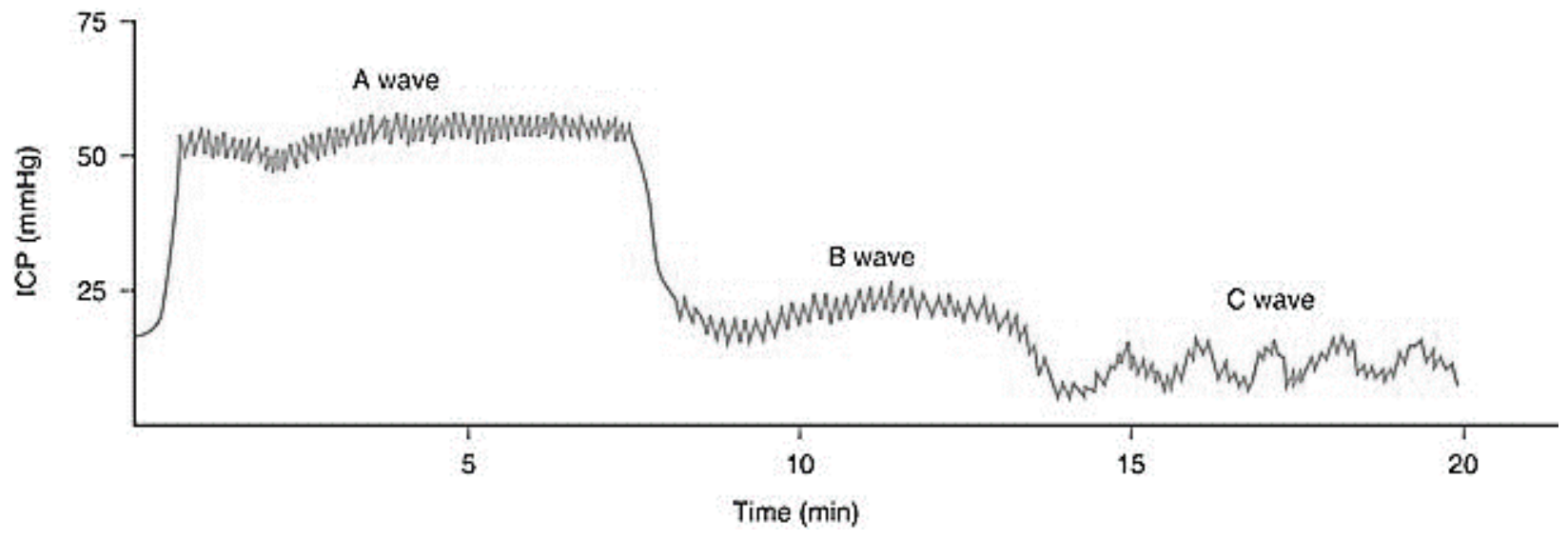

Figure 2.1 ICP A Wave, B Wave and C Wave 


\subsection{Wavelet analysis}

Wavelet analysis was broadly applied for decomposition of ICP waveform and separation of its elements; which allows the non-stationary nature of the signal be considered in the analysis [43], [67], [41]. Analysis, explanation, and interpretation of ICP signals is usually associated with error. The present techniques have some shortcomings is identifying discrete ICP signal peaks of unbalanced waveform. Moreover, hardware connections and activities causing volume variations from any signal contribute in generating noise in ICP signals which can introduce error and make the ICP signal less reliable for medical intervention [68] [69]. To overcome this challenge, it is suggested by several scholars to identify the ICP peaks which give insight for better understanding and assessment of waveform and enhance the quality of ICP signal [63][70][71][72].

Tsui and his co-workers developed a neural network model using wavelet analysis for multistep ICP prediction [51]. Analysis of the variations in ICP sub-peaks, features related to ICP amplitude and also duration of the sub-peaks is also a practical method suggested in Ref. [71] which is of crucial important for clinical applications [10]. Kashif et al. [73] proposed a cerebrovascular function model which utilized the arterial blood pressure (ABP) waveform analysis and cerebral blood fellow velocity $(\mathrm{CBFV})$ to estimate the model parameters.

Wavelet analysis is capable of reconstructing various linear and polynomial function shapes (i.e., rectangle, triangle, second order polynomials, etc.) [74] that is not possible using Fourier analysis. Wavelets analysis could consequently eliminate the ICP noise more efficiently compared to Fourier transformation filtering. Fig 2.2 demonstrates the non-stationary characteristic of a sample ICP within 1200 seconds. Fig 2.3 corresponds to the wavelet analysis of the same signal duration and as demonstrated, wavelet reflects a weak signal power within the first $400 \mathrm{~s}$. As shown in Fig. 2.2, the signal peak occurs between $t=550$ to 620 seconds, which is also highlighted in red by wavelet analysis of Fig. 2.3. Signal level decreases between $t=620 \mathrm{~s}$ to $t=820 \mathrm{~s}$ and subsequent slight increases shown in Fig. 2.2, are highlighted in Fig. 2.3 using wavelet power levels varying between yellow and green, which is followed by the later peak around $1100 \mathrm{~s}$. 


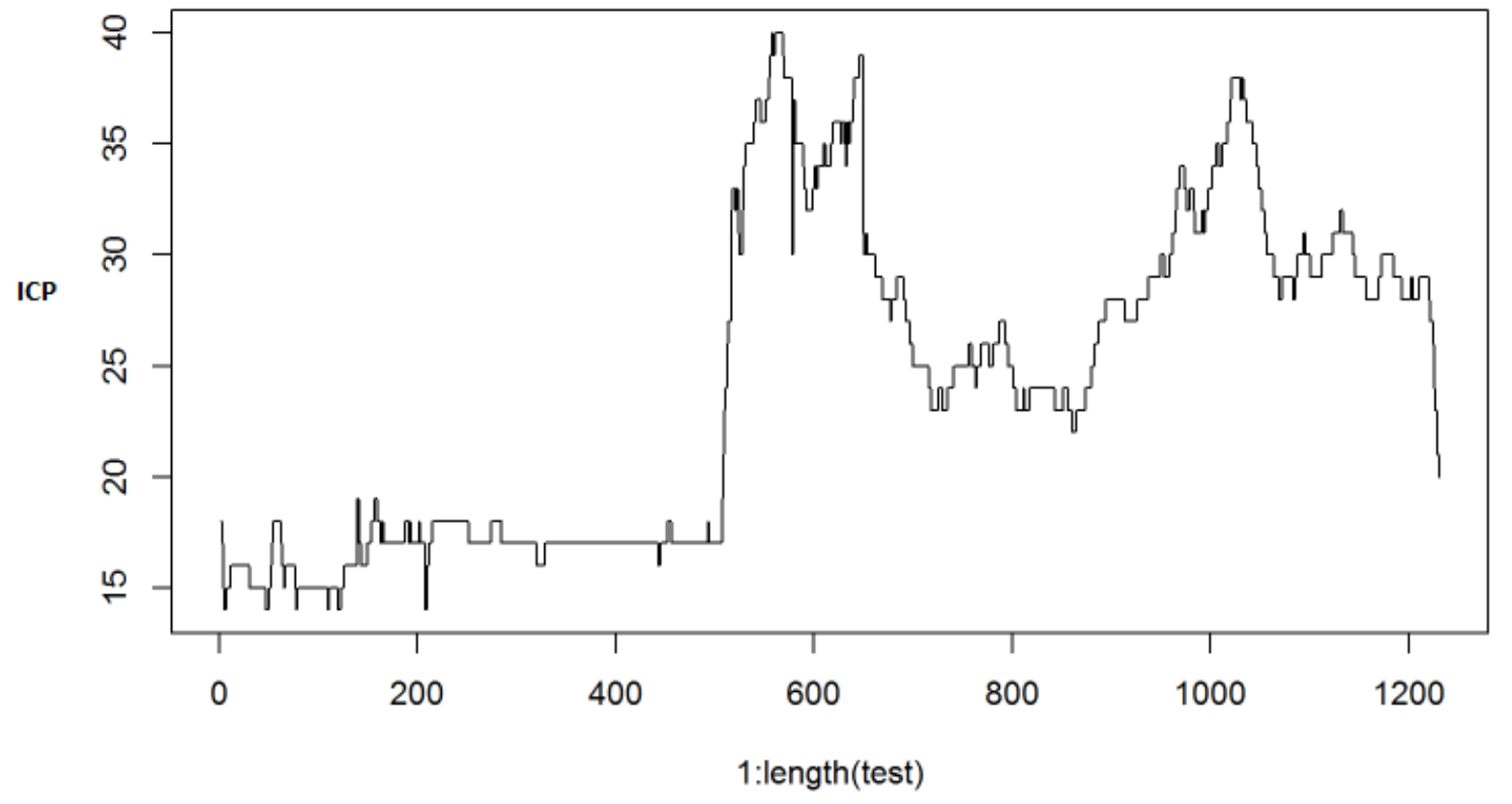

Figure 2.2 a sample of ICP signal of a traumatic brain injured patient over an episode of 1200 seconds

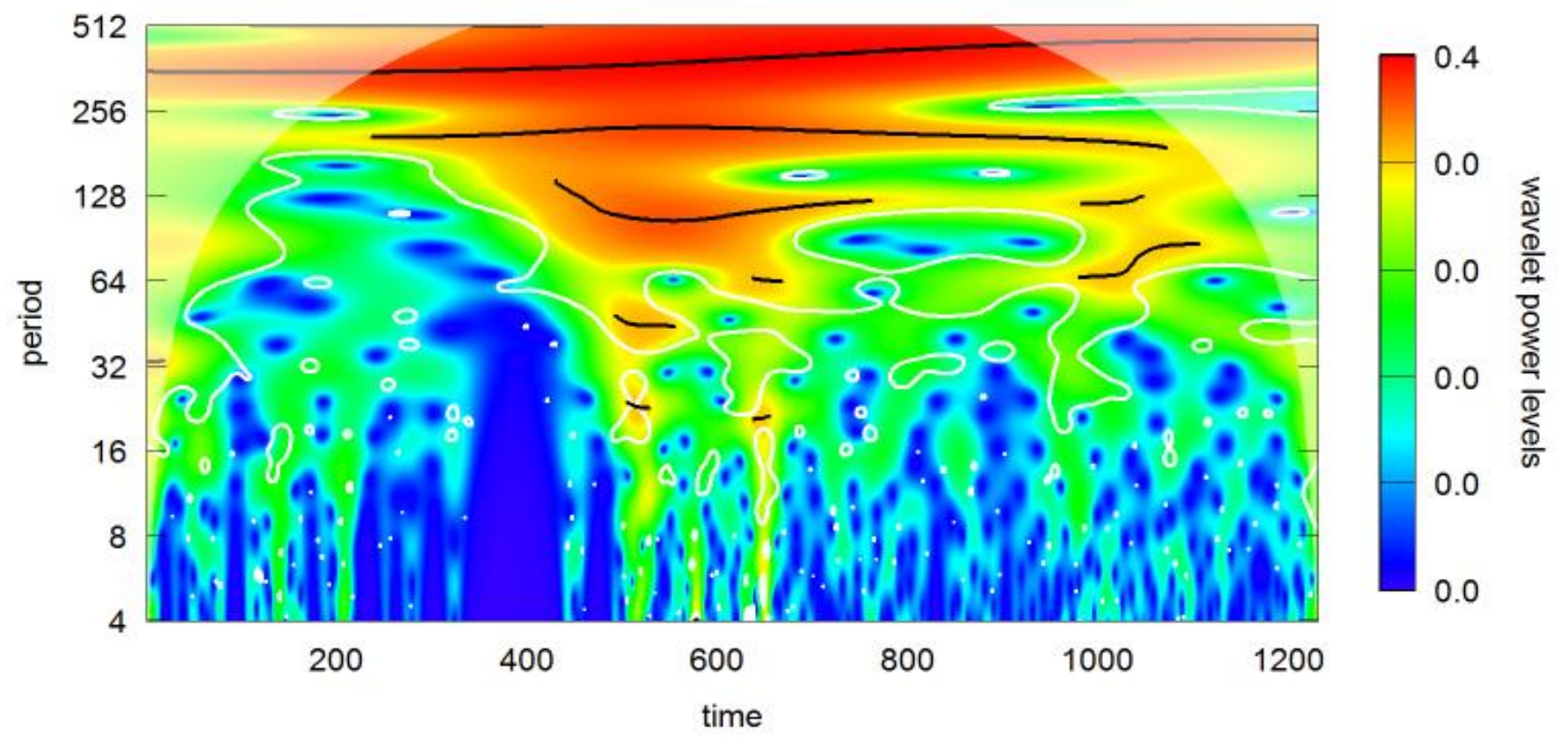

Figure 2.3 wavelet power levels corresponding to the same patient in Fig. 2.2 


\subsubsection{Spectral analysis}

The analysis of time series, also known as signal analysis, is a fundamental challenge for scientists [75].

The spectral analysis works based on measurement of amplitude vs. frequency input signals [76]. In order to analyze the frequency domain of ICP one needs to realize the harmonics component of the ICP signal [34]; which are acquired via a mathematical transformation (e.g., FFT) that can separate and identify each waveform from the total spectrum, known as spectral analysis [77]. Spectrum and vector signal analyzers are applied for time series measurements [78]. FFT was applied for a long time to map the data from time to frequency domain to assess ICP signal and explain brain compliance [73] which was found to be more trustworthy compared with mean ICP

analysis [41]. Spectral analysis is capable of analyzing wide range of frequency and bandwidth, though it is neither as appropriate for measuring transient events nor phase measurements compared to wavelets analysis [10], [79].

\subsubsection{ICP fractal-based analysis}

Fractal analysis is a proper method to study the correlations in the ICP signal during long time periods. The term "fractal" which is widely used in neuroscience was first used by Mandelbrot to refer to the irregularity and complexity of features and objects [80]. Fractals are described by an interesting property known as the self-similarity; i.e., the subunits resembling the bigger scale structure [81]. In other words, the observed features at a superior enlargement level theoretically resemble the ones at a minor level. Considering this, the focus of fractal analysis is to determine the self-structure, self-similarity, and eventually pattern recognition of ICP waveform. Burr et al. [82] examined the possibility of applying detrended fluctuation analysis (DFA) to ICP signals collected from TBI patients and correlated the derived elements to patients outcome. They discovered that the DFA smaller intercept and scaling exponent are accompanied with the negative TBI patient outcome [10] [88].

Fractal analysis has some advantages over the other techniques; i.e., this method is widely practical for signal analysis as it can identify similarities among different signal segments which 
help improve the signal predictability. Moreover, the fractal pattern is preserved once a fractal object is projected to different dimension order. Furthermore, the expansion or contraction of the ICP signal will not change the shape of signal in any dimension. Despite the benefits of this methodology the relation between highly complex physiological responses and high fractal dimension requires further investigation [83].

\subsection{Hidden Markov model}

Hidden Markov model (HMM) could be visualized as a finite state process, known as a relatively simple modeling method for sequential data [84]. Markov process is known as stochastic process that satisfies Markov property names as "memorylessness". HMM is an appropriate option when the observational data is available but the transient states are unknown [84]. Despite the advantages of HMM in ICP analysis, it requires noticeable amount of data for training and also a priori information of the model topology [85].

Rezek et al. [86] showed that HMM can be used together with some learning methods and utilized HMM to different biomedical signals such as the periodic breathing, electroencephalogram (EEG) and the RR-interval series. Novak et al. [87] classified real ICP data by applying a clustering algorithm on continuous HMM and demonstrated that up to $80 \%$ accuracy performance could be obtained. Jordan et al. [88] investigated the time series model using a decision tree with Markov chronological structure to describe the use of HMM for time series analysis.

\subsection{Kalman filter}

A Kalman filter is a tool to obtain the parameter of interest from indirect and unclear observations[89]. Kalman filter processes the incoming data recursively and is capable of minimizing the mean square error of the predicated factors associated with Gaussian noise distribution [90]. Considering that the analysis of signals involves past, present and forthcoming signal values [91], Kalman filter only requires to keep the previous state and operate fast to predict the prospective values [92]. Kalman filter can also be applied on ICP waveform and analyze the information despite the existing entropy [93]. Kalman filter is a practical and optimal tool for 
accurate modeling of various linear systems [94]. Kalman filter is also useful for online real-time processing that can be simply implemented.

Nonlinear systems can also be processed using extended Kalman filter that linearizes the predictions and measurements of the average signal value [95]. Despite the advantages of the Kalman filter model, the ultimate error levels can be fairly high, once the model does not function accurately; therefore, user need to assess the advantages and error of this methodology while applying that for cases with few random variables [59].

Hu et al. utilized physiological variables (i.e., CBFV and ICP) to anticipate unseen ICP episodes using a nonlinear Kalman filter and coupling the estimators with the input and output configuration [96]. Aboy et al. utilized dual Kalman filter to develop a practical algorithm that applied ICP power spectral density collected from TBI patients [97]. Wan et al. developed a nonlinear technique named as dual extended Kalman filtering (EKF) and applied that on time domain to eliminate the noise from voice signal and proposed that for medical signal applications [98].

\subsection{Machine learning techniques}

The state of the art machine learning techniques applied on ICP will be reviewed in the present section. Machine learning methodologies enabled computers to enter smart level of self-learning [99] which is predicted to lead into significant improvement in medical intervention techniques. Majority of the methods and studies reviewed in this section focused on the ICP classification, clustering and prediction.

\subsubsection{Logistic regression and artificial neural networks}

Predictive models are generally constructed using realistic data from the past experience and are critical tools for medical diagnosis and prediction. An important step of predictive modeling is the required data preprocessing needed to be done prior to machine learning, which is sometime time consuming. Basically, the underlying patterns in the realistic data will be identified using the mathematical algorithms implemented using the knowledge of computer science [100]. 
Artificial neural network is a method of machine learning capable of predicting the future output signal based on the properties of the learned input signal which were recommended for medical applications since 80's. Such a system is developed to recommend the most appropriate intervention based on the medical diagnosis [60]. Artificial neural networks for ICP prediction are developed to assess the behavior of ICP deterministic components and predict the output ICP signals [64][61]. The simple recurrent neural network, recommended for ICP prediction of patients with head injuries are capable of identifying critical deterministic components [101].

Logistic regression utilizes a set of independent variables to anticipate limited outcome variables; implying that inaccurate selection of the independent variables results in extremely poor predictive values [102]. On the other hand, not only accurate independent observations are required for proper operation of logistic regression model, but the model may also overfit the training data [103]. Logistic regression model is capable of predicting two explanatory or dependent variables that can be either be ordinal, dichotomous or continuous [104]. The logistic regression model remained preferable compared to Chi squared distribution or Fischer's exact test of independence techniques [105].

Swiercz et al. [106] compared neural network and linear autoregressive for ICP prediction and discovered that although linear autoregressive model operates better for prediction of future ICP in time range of 2 to 3 minutes ahead, neural network perform better in the prediction of 5 minutes ahead or more [106]. Dreiseitl et al. [100], described the applicability of logistic regression and artificial neural network models for biomedical data classification and introduced the statistical pattern recognition as the mutual step in logistic regressions and artificial neural networks models. They also compared these two models with various other classification algorithms and showed how to construct and assess these two models.

Fanelli et.al [107] developed a physiologic model to predict ICP signals based on regression correlation between ICP and ABP and CBFV and tested the model using 20 different datasets recorded from three sever care unit patients. They compared their predictions with invasive ICP recording and reported estimated ICP values with average error and standard deviation of -1.12 and $5.56 \mathrm{mmHg}$, accordingly [107]. Guiza et al. [108] also developed a predictive model for ICP using logistic regression and evaluated their model using ten repetition of five-fold cross validation technique and obtained an acceptable discriminative ability utilizing relatively few body signals 
[108]. These studies indicate that autoregressive model is a suitable method for prediction the ICP for the upcoming few minutes; however, neural networks predict the future ICP more accurately when it comes to ICP prediction for farther and longer time periods.

Hüsser [2] developed a regressive analysis model ( for non-invasive prediction ) as well as SVM and ensemble extremely randomized tree utilizing ICP and physiological signals. Hüsser [2] utilized body signals in different time scales to introduce complex features and analyzed signals in multi scale time series. He trained and tested his models using MIMIC II dataset and BrainIT dataset, respectively; and reported an AUROC score of 81 utilizing 10-fold cross validation method on the classification method and an MSE of $3.84 \mathrm{mmHg}$ on the regression model.

The recent progress and advantages achieved by using neural networks, compared to preceding algorithms, attracted lots of attention and developed broad practical applications in medicine [46],[62]. While the old statistical techniques reached their inherent capability limits due to their shortcomings in modeling the non-linear dynamic process as reflected by ICP waveforms, neural networks were introduced a promising method for multi-step ICP prediction in studies conducted by Tsui et al. [51].

Compared to logistic regression modeling, artificial neural networks, operates significantly better in identifying nonlinearities among the dependent and independent variables, needs less training whilst having various training algorithms and works better in detecting the interaction among the independent variables [109]. Despite its advantages, artificial neural network also suffers from few shortcomings: i.e., expensive computational cost (extended computations), possibility of model overfitting, empirical nature of model development and black box characteristic [109].

\subsubsection{Support vector machines}

Support Vector Machines (SVM) known as the algorithmic application of statistical learning theory are utilized for constructing classifiers for feature space [110]. To classify features accurately SVM can generate a classifier for a known pattern classification problem. SVMs can construct efficient separating borders among data [111] [112]. SVM can construct a hyperplane or sets of hyperplanes in high or infinite dimensional space for classification, regression analysis or other tasks such as outlier detection. Support vector machines got more attraction in medicine 
compared to other machine learning algorithms [100]. SVMs were broadly investigated and applied for classification and function approximation.

Development of classifiers is generally through either of the following two approaches: 1- a parametric approach 2- Non-parametric approach. Parametric approach operates based on the assumption of data distributions whereas nonparametric approach does not need the data distribution to be assumed [113].

SVMs and artificial neural networks are considered as nonparametric classifiers which can be trained utilizing sample data. Afterwards, classifiers determine the decision functions that determines to which class input belongs [114]. SVMs operate with a regularization parameter and the solutions converges to global minimum [115]. The most significant shortcoming of SVMs relies in selection of kernel [116]. Moreover, the training and testing size and speed is also another issue reported for this method [116]. Chen et al. [117] used SVM and integrated different sources of data such as injury scores, demographic data and CT image data to identify the correlated parameters affecting ICP and validated their model utilizing cross-validation for feature selection and tuning parameters.

\subsubsection{Naïve Bayes}

Naive Bayes classification method which has commonly been applied in medicine was developed as a technique to improve medical intervention decision. Naïve Bayes method is capable to handle cases where there is strong independence amongst the features and is developed based on application of Bayes theorem. Naïve Bayes model has been utilized by several researchers to predict patients' outcome. Kazmierski et al. applied Naïve Bayes model for assessing cancer relapse subsequent to radiotherapy and reported an accuracy of $84 \%$ in classification and sensitivity of $80 \%$ [118].

Klement and his co-workers developed a prediction model on CT imaging data using Naïve Bays technique and reported $82.8 \%$ sensitivity of the model [119]. Wei et al. also utilized Naïve Bayes technique to develop a model for patient outcome prediction from genome-wide measurements and reported the AUROC of 0.59 achieved by Naïve Bayes algorithm [120]. Despite its reliance on the assumption of strong input independencies, Naïve Bayes is a relatively popular method due to its 
capability to estimate the model parameters using small amount of training data and easy implementation [121].

\subsubsection{Decision tree}

Decision tree is considered as a helpful decision making strategy utilized for research analysis. This technique is useful in showing the possibilities resulting from a series of decisions and picking the suitable one. Decision three is consisted of some branches, lead nodes and the root [122]. The base of decision tree is the root, which along with the leaf nodes embrace the criteria/questions that is to be met/answered; and the flow starting from the question to the answer is shown through the connecting arrows also called the leaf nodes [111].

The J48 decision tree constructs an initial decision tree using the available training data to classify a new item; which was utilized by Galenao et al. to study the ICP signals and resulted in $73.33 \%$ accuracy [123]. Scalzo et al. [122] developed a highly randomized decision trees, utilizing ICP based morphological features, to classify TBI patients and predicted ICP signals accurately. The comparison conducted by Scalzo et al. demonstrated generalization performance using linear and nonlinear methods [122].

Compared to other techniques, decision trees can be understood and implemented easier specifically in case of few decisions and outputs and generally is considered as a less complicated modeling method [124]. However, larger decision trees involving few dozens of nodes can possibly get complicated and less practical for decision-making.

The more decisions there are in a tree, the less accurate any expected outcomes are likely to be. The decision tree often predicts the expected consequences of each decision appropriately and assists to improve the optimum decision-making by weighting potential consequences of decisions. Despite the several advantages of decision tree, one of the shortcomings is that the tree is developed based on expected outcomes [125] [115]. 


\subsubsection{Wavelet clustering techniques}

Clustering is a technique to detect dense areas of data population and categorize them for further application in data mining and optimum information restoring. An optimum clustering method is ideally independent from data noise or outliers as well as neutral to the order of input data. Wavelet clustering techniques is broadly utilized for anomaly diagnosis [126]. Wavelet transform and super paramagnetic clustering technique were utilized for unsupervised spike detection by Quiroga et al. [127].

Ghosh-Dastidar and Adeli [128] used speech and volume data and developed a model utilizing statistical cluster analysis and wavelet-based signal analysis for incident detection. They trained the neural network for pattern detection using the clusters and reported a $100 \%$ successful incident detection rate during 35.6 seconds, associated with the false alarm rate of $0.3 \%$. Sheikholeslami et al. [129] proposed a clustering method based on wavelet transforms, to meet the characteristics of an optimum clustering mentioned at the beginning of this section named Wave-Cluster. They applied the clustering on the multiresolution characteristics of wavelets and demonstrated the efficiency of Wave-Cluster for analysis of time series [129]. Vlachos et al. developed a model for analysis of time series using k-means clustering with some changes, and named that the new model of k-means clustering set of rules for time series that operates based on the multiresolution property of wavelets [130].

To predict the epileptic seizure from EEG signal, Geva and Kerem [131], proposed a novel identification method tested on 25 rats applying wavelet analysis to extract wavelet features and fuzzy clustering to cluster wavelet images. The methodology succeeded in identifying several states of interests such as "sleep", "resting", "alert and active wakefulness" as well as the seizure.

In another study, an emotion recognition system proposed by Murugappan et al. [132] using discrete wavelet transform for feature extraction together with three statistical features extracted from EEG signal, the authors used fuzzy C-means clustering for emotion recognition. The study confirmed that wavelet based extracted features from EEG signal together with statistical features were able to conduct human emotion detection.

In another study $\mathrm{Xu}$ et al [133], proposed clustering physiological signals to detect human

stress level. In this study K-means clustering algorithm was used and the result indicated a 
significant improvement when compared to approaches not using clustering. In another study by Faust et al. [134] a review of wavelet techniques used for seizure detection and epilepsy detection on EEG signal was conducted and the most effective method was introduced to be integration of wavelets, nonlinear dynamics and chaos theory together with neural networks for epilepsy diagnosis based on EEG signal. In another study by Melek et al. [135], systolic and diastolic blood pressure and heart rate as physiological signals have been used as time series data to enable trend detection with wavelet techniques, statistical, regression analysis and fuzzy clustering. Moreover Li et al.[136], experimented modeling of cardiovascular physiological signals based on wavelet transformations and adaptive Hermite decomposition functions, the authors reported that orthonormal wavelet transformations are computationally effective and adaptive wavelet basis functions are able to model cardiovascular physiological signals with certain cost of missing orthonormalities.

In another study by Schwarz et al. [137], wavelet based clustering MRI modeling and data driven signal modelling of MRI have been compared and reviewed and the authors concluded that wavelet clustering is a suitable technique to distinguish patterns of pharmacologic MRI responses and the methods provides a good sensitivity when conducted on highly dynamic signals changes.

Finally wavelet based fuzzy clustering has been experimented by Chao et al. [138], for determining groups of similar patterns as a novel method having the advantages of being fully automated, adaptive to the diversity of physiological signals, effective visualization and less sensitive to noise and artifacts. The methodology was noted as a superior method in long-term polysomnography analysis.

To the best knowledge of the author, there are no previous studies applying "clustering of the wavelet images of the correlated physiological signals with ICP to understand changes of ICP in traumatic brain injured patients" similar to the conducted unsupervised modeling in this thesis; and the preceding literature review was provided to discuss the different aspects and history of this technique. 


\subsection{Summary}

Clinicians need to investigate broad range of physiological parameters to diagnose the symptoms and pick the most appropriate treatment for brain injured patients. ICP waveform analysis is the most important step in medical intervention of patients with head injury. ICP analysis provides an exquisite insight into intracranial/cerebral dynamics, helpful to detect patients having low cerebral adaptive capacity; who may also suffer from intracranial hypertension that potentially results in permanent secondary brain injury.

Considering the importance of intracranial hypertension, the intracranial pressure data recorded in various studies were utilized in modeling to predict the episodes of elevated ICP. However, many of these models are found to be expensive, invasive and difficult to maintain and yet not enough having predictive value which reflects the present demand in further modeling of ICP. This thesis performs further investigations for prediction of ICH noninvasively by including more channels and defining related predictive components from body signals.

The purpose of the thesis would be to conduct modeling normal/Intracranial hypertension state of ICP based on correlated physiological signals with ICP in the feature space only and build a predictive model to map correlated body signals with ICP, which is missing in the literature. The ICP models developed in this thesis took medical considerations into account and also answered the fundamental question about the presence of a structure within body signals that could reveal the changes in ICP. 


\section{Methodology}

\subsection{Introduction}

In this chapter, a number of significant correlations between some of the vital signals of the TBI patients and ICP are investigated using statistical and information gain analysis, which use the vital signals as potential predictors of ICP status. The scope of our proposed work is the application of data mining, statistical approaches and machine learning to find predictive features of ICP in physiological signals. Moreover, this chapter presents two methodologies (Classification and Clustering) for the aforementioned problems.

\subsection{MIMIC II waveform dataset}

The public Multi-parameter Intelligent Monitoring in Intensive Care (MIMIC) II database [139] contains multiple physiological signals as well as the time series of vital signals on the patients corresponding to 25,328 patients hospital stays. The data is collected at the Beth Israel Deaconess Medical Center located in Massachusetts. The target dataset of interest in this thesis is MIMIC II Waveform dataset, which contains samples of ICP signal. The recordings are all anonymized, and they are publicly available for the research purposes and incorporate recordings of various physiological signals, which contains arterial blood pressure (arterial, venous, and other), respiration and fingertip photoplethysmogram (PPG), etc. vital signal time series collected as numeric time series often includes respiration rate, $\mathrm{Sp02}$, systolic, diastolic and mean arterial blood pressure, heart rate, etc.

The data collection frequency differs depending on vital signals time series to waveforms. The numerical vital time series are collected at $1 \mathrm{HZ}$ and the waveforms are collected at $125 \mathrm{HZ}$. The ICU staff has decided which signals need to be collected from the patients, however the majority of recording do have vital signal time series collected from the patients. Thus, records include samples of signals which have been considered clinically relevant during the time of treatment. In this study all of the records were filtered initially only based on availability of the column name 
ICP signals and 218 patients are obtained and only 97 patients which contained ICP in at least $50 \%$ of the total recording time have been included in the analysis. The patient population have ICP recording in various time intervals, which has been clinically determined for how long ICP monitoring should be conducted for each patients.

The waveform dataset has some matched dataset where some clinical data of the patients such as age, sex, duration of stay, etc. are found. The data is initially merged on patient ID to have a better picture of the patients for analysis. The clinical information of the patients having ICP monitoring is not broadly available. In more than $70 \%$ of the records of patients having ICP records as other vital time series, the "matched dataset" does not supply clinical information of those recordings suitable for this analysis.

Individual statistics on the availability of signals in the selected 97 records are shown in Fig. 3.1 and later demonstrated in table 4.4. The summary of different signals and their description on MIMIC II website is as follows [139]; Pulse, ST III, ST V, RESP un-calibrated respiration rate, estimated from thoracic impedance, ART arterial blood pressure (invasive, from the other radial artery), CPP cerebral perfusion pressure CVP central venous pressure, ICP intracranial pressure, BP blood pressure (systolic, diastolic, and mean), HR heart rate, PVC Rate, SpO2 oxygen saturation (from fingertip plethysmography) The following signals shown in Fig. 3.1 were available for the aforementioned 97 patients who have been based on the criteria of existence of ICP records in at least $50 \%$ of the total recording time. 


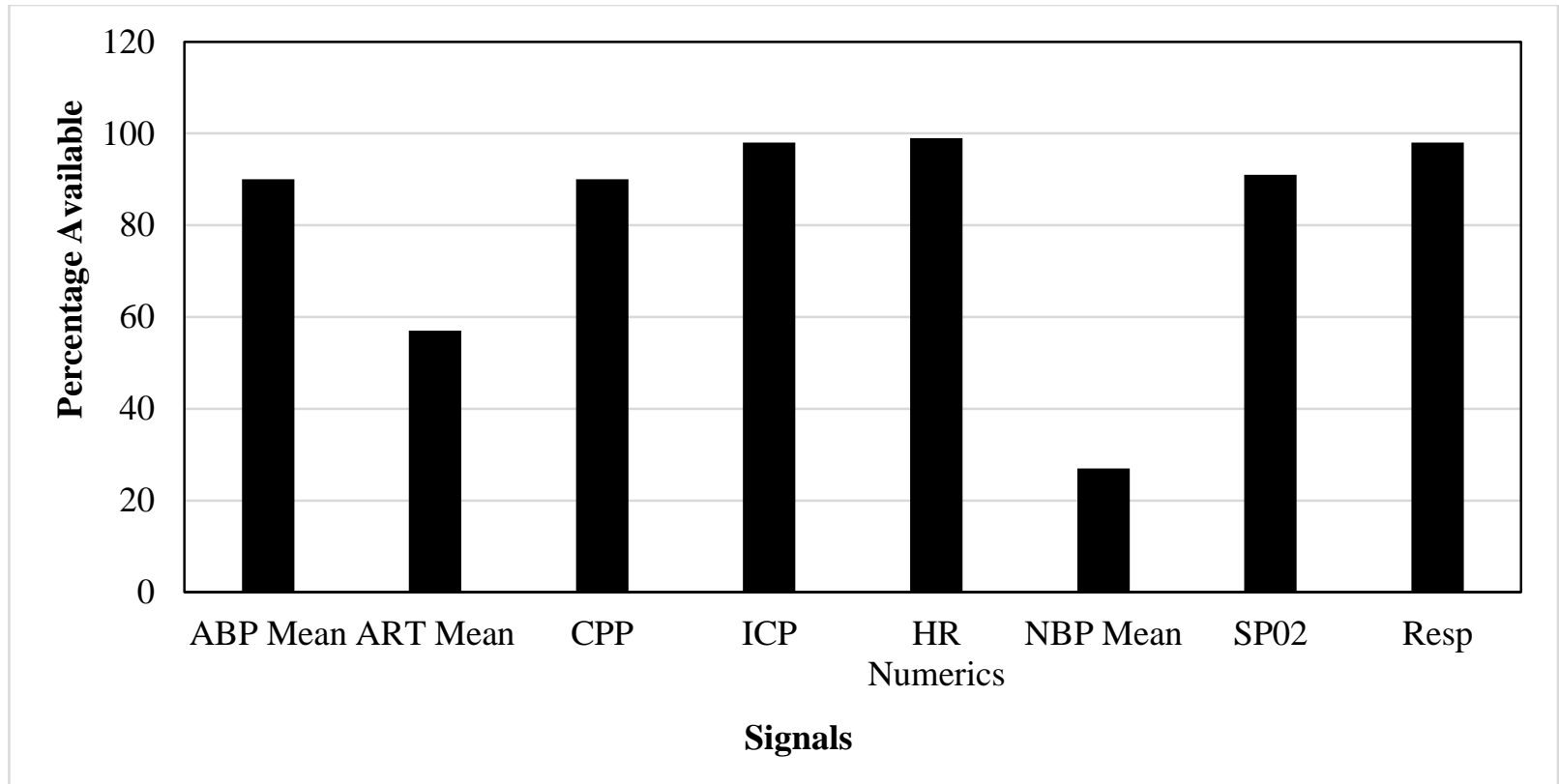

Figure 3.1 Signal availability statistics for the MIMIC II data samples

\subsection{Permission of access}

MIMIC II waveform datasets are available publicly for researchers. On the other hand, accessing the BrainIT dataset required writing a proposal to be assessed. So a proposal was written and it was reviewed by 21 neurosurgeons who had created and maintained the database. Once the proposal received the permission, the physiological signals with extra clinical data available such as daily observations, demographics of the patients, ICU-monitoring, Lab results, Neurological status, other clinical events such as x-rays, physio, Doppler, operation, blood samples, etc. and the surgery results were granted access to.

\subsection{BrainIT dataset}

The BrainIT dataset [140] is gathered through many hospitals and across 22 clinics based in Europe. The collection consists of demographic information (static information such as age, gender etc.) as well as the physiological information. The demographic table is the master table and each patientID in it maps to one or more rows in each of the other tables. 
The physiological table has the most entries (>2 million rows) which has the data collected at $1 \mathrm{HZ}$ from 261 patients. The BrainIT dataset includes the following information [140]; Daily observations, Demographics, ICU-Monitoring, Lab-results, Neurological-Status, Other Clinical Events, ICP (mean, systolic, diastolic), Surgery Information, Physiological Information, Glasgow comma scale, History of previous neurological disfunction.

As per the BrainIT core dataset operation manual, the data frequencies is described as follows; Once only, in which the demographic and clinical data was collected once for each traumatic injured patient, Periodic Time-series, collected through physiological monitoring at specified time intervals, Episodic time-series, in which the information was collected at different unpredictable time spots; i.e., blood gases, nursing comments, GCS scores (intensive care management), Episodic time-series, in which the information was collected at different unpredictable time spots; i.e., drug infusions, drug bolus's, changes in ventilation settings (Secondary Insult Management). In this thesis, the physiological information and the ICP information are the desired information, which were used to evaluate the generalization ability of the non-invasive predictive model.

\subsection{Filtering the dataset}

The preliminary step utilized in data analysis was to collect the data sets, initially filtering based on availability of ICP signals. The filtered data approximately corresponds to 67 days of ICP signal measured at $125 \mathrm{~Hz}$ or $1 \mathrm{~Hz}$ and vital signals measured at $1 \mathrm{~Hz}$ and 86400 records were collected per day. The input samples included in this analysis are only dealing with ICP collected at $1 \mathrm{HZ}$. In the initial pre-processing step intracranial hypertension is defined as an elevation of the ICP over $20 \mathrm{mmHg}$ continuously elevated for the length of 30 minutes [57]. Once episodes of increased ICP are collected, the corresponding vital signals to those records are collected as well.

In the next step, ICP recordings have received a label in such a way that ICP less than 20 $\mathrm{mmHg}$ have received the "normal ICP" and ICP values greater or equal to $20 \mathrm{mmHg}$ continuous for 30 minutes have received the "high ICP" label. Moreover, all of the records were scanned manually to ensure the patient ID is not redundant and different records belong to different patients and no two records correspond to the same patient for different reasons of hospitalization. 
As part of the pre-processing, it was observed in the dataset, that the intracranial hypertension samples and normal samples were unbalanced on the MIMIC-II dataset, which was considered in training and evaluating the classifier. For this purpose, the class labeled balance setting was applied in the configuration of neural network as shown in configuration of neural networks in table 4.11.

\subsection{Data visualization}

Figure 3.2 demonstrates the scatterplots of mean ICP vs mean blood pressure before cleansing and noise removal which demonstrated a linear correlation. The mean blood pressure is the main

channel of the study. In addition, Fig 3.3, demonstrates the parallel coordinates of correlating body signals with ICP in 500 samples. The figure shows the trend of normalized mean values of the correlating signals with ICP in different ranges.

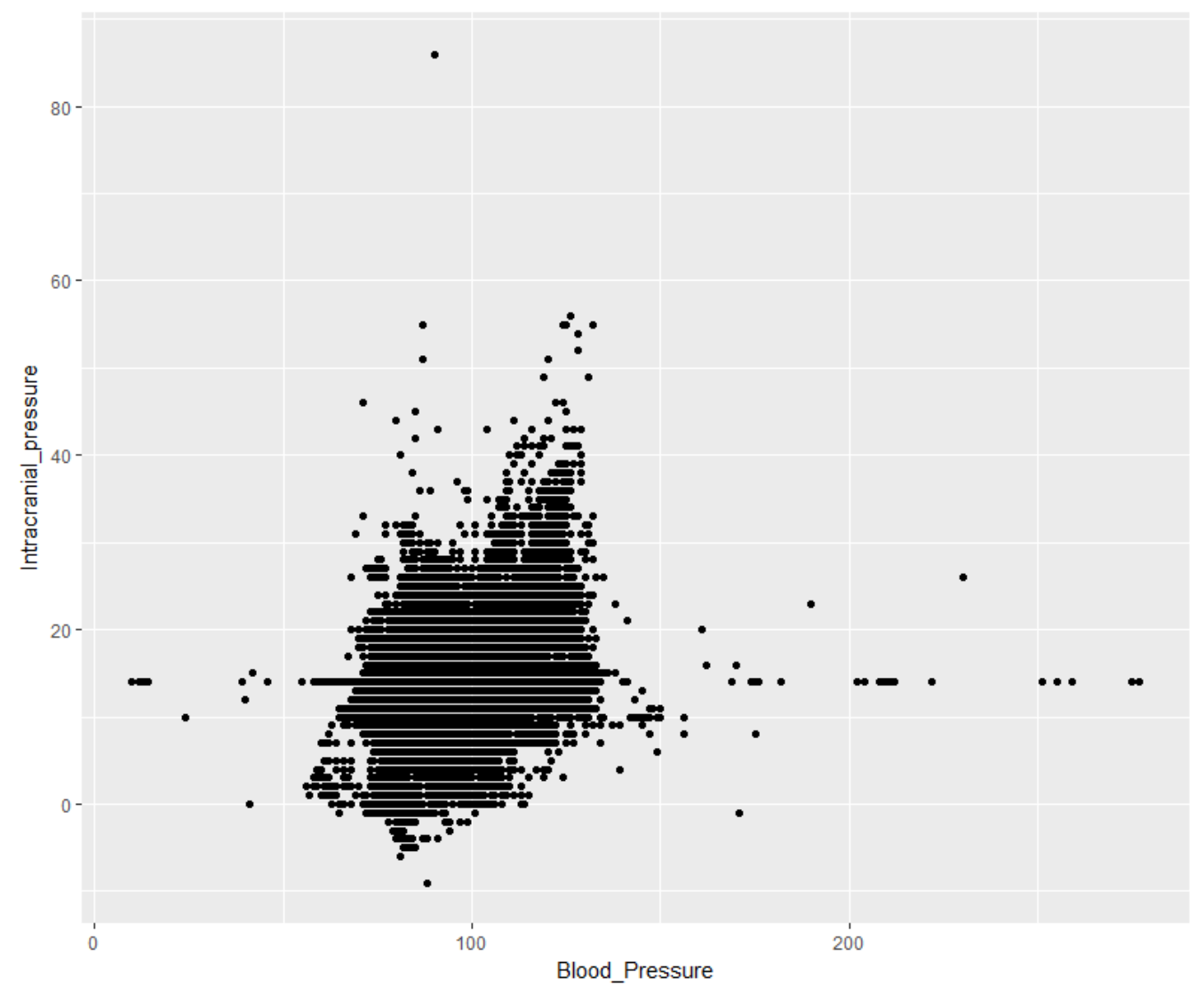

Figure 3.2 Scatterplot of Intracranial Pressure vs. Blood Pressure 


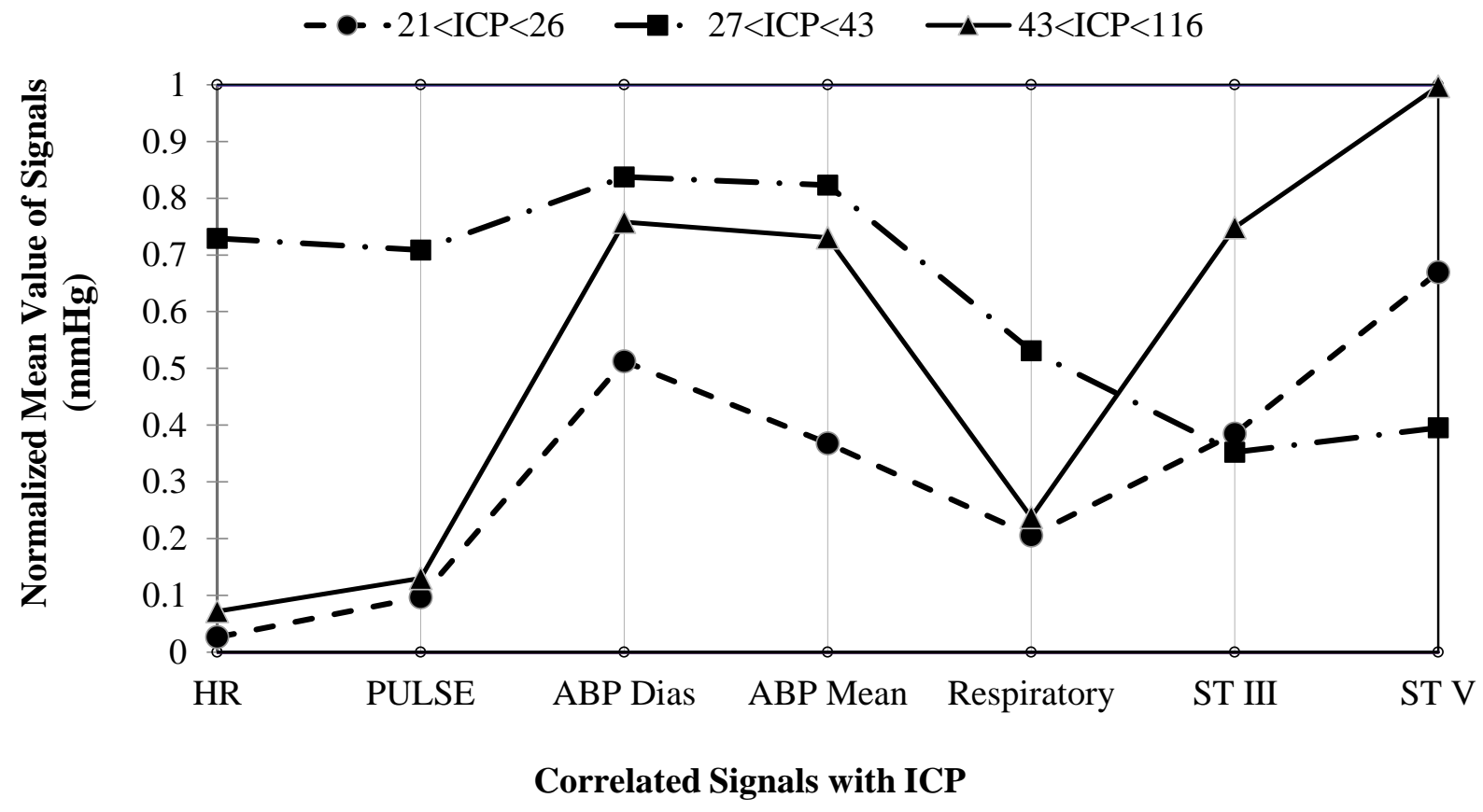

Figure 3.3 Parallel Coordinates of Correlating Body Signals with ICP

\subsection{Information extraction}

To extract the episodes of intracranial hypertension in the chosen dataset, an algorithm was developed and coded in Python to return the increased ICP and its subset in the existing dataset (Appendix B). The medical definition of ICH and medical experts' advice on ICP duration were considered for this purpose. Duration of the ICP hypertension was set to 30 minutes and the ICP threshold was defined to be $=>20 \mathrm{mmHg}$. The algorithm in pseudocode is as follows:

1. Define a threshold as ICP $>=20 \mathrm{mmHg}$

2. Detect where ICP >=20 mmHg exists either 30 mins forward or 30 mins backwards, if true mark the line numbers in new column valid_highICP

3. Else reset data array, and set previous ICP as false prev_data $=$ False full_array $=[]$ 


\subsection{Data cleaning}

The commonly observed low signal to noise fraction as well as unwanted artifact pollutions are some of the concerns in analysis of physiological signals. For example, sensors and the analog to digital converting devices may cause high-frequency noise in ICP signals [2],[141]. On the other hand, patient movement and coughing may cause low frequency noise. Moreover, an unconnected/detached sensor may continue to send signal for a limited period. To improve the quality of any signal, it is important to conduct artifact and noise removal [5].

The artifacts associated with ICP signals and other types of signals such as diastolic blood pressure, mean blood pressure, etc. were removed using the followings steps, as was generally suggested in ref [2] with some modifications.

Initially, it was evaluated either the recorded ICPs could be used or should have been discarded. To implement this step, the ICP signals with greater than $30 \%$ of absent or corrupted data points were tagged as "invalid". Corrupted data points were defined as the abnormal data points that were beyond the physiological range. As the result, the patient population were reduced down to 91 people.

The high frequency noise in ICP signals utilized in the present study were removed using the "Zero-phase digital filtering" implemented by Matlab's filltfilt function. This filter could preserve features of any signals at the time they occurred in the presence of noise. Figure 3.4 illustrates an example of 392 seconds of original ICP magnitude plots and Fig 3.5 demonstrates the corresponding cleaned (filtered) ICP magnitude plot. 


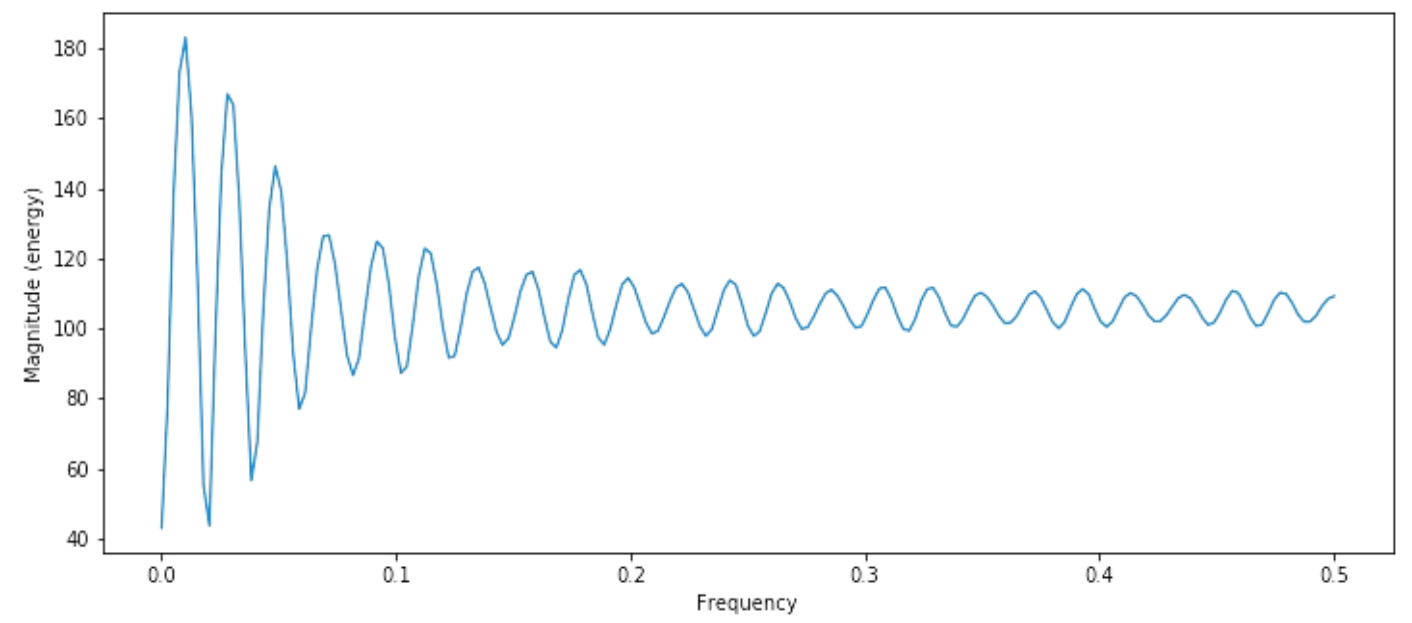

Figure 3.4 Original ICP magnitude plot

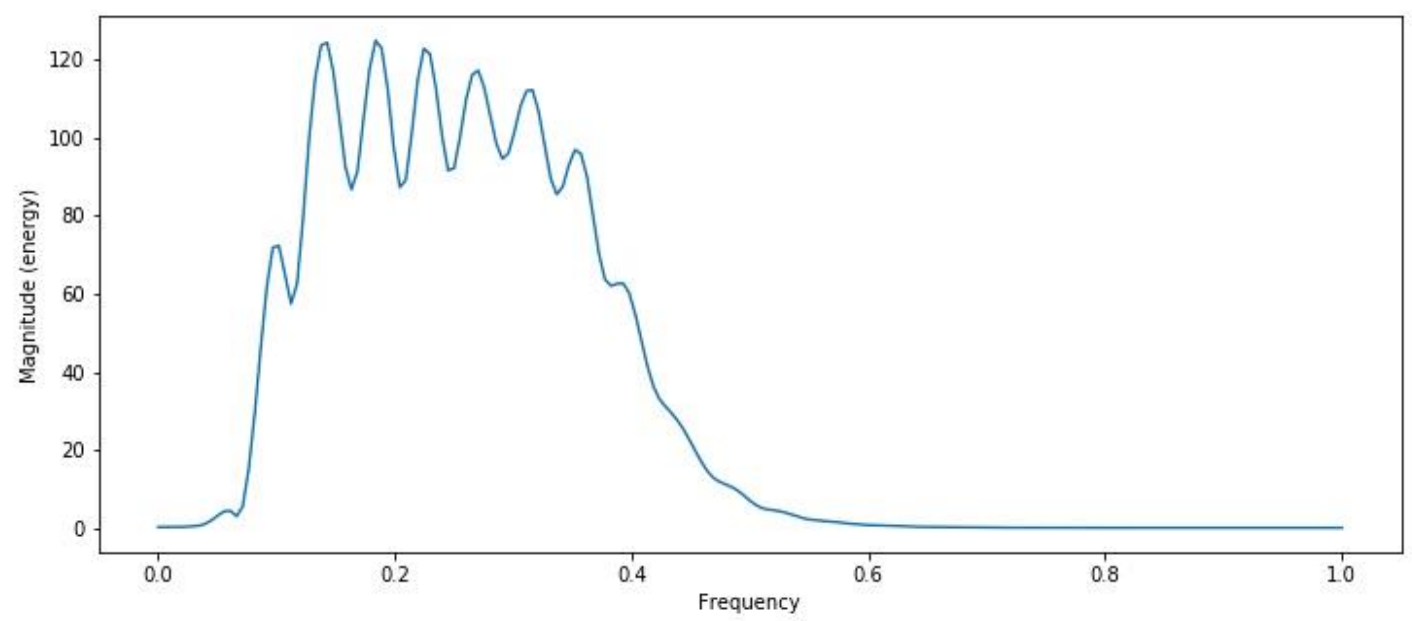

Figure 3.5 Filtered ICP magnitude plot

Multiple imputation methods [142] was used in the present research to fill in the missing records with attainable values according to the pattern of the data. Multiple imputation methods evaluate the pattern of records and based on likelihood (probability) of occurrence of the data, finds the best match and replace missing values. Replacement is executed through an iteration loop to discover the best fit. IBM SPSS Statistics version 22 [143] that supports multiple imputation methods was utilized for this purpose. 


\subsection{Finding correlation: Pearson correlation coefficient}

A Pearson correlation was used to determine the correlation among 11 biomedical signals and ICP values collected from 91 TBI patients having intracranial hypertension crisis on their first 12 hours of hospitalization. Cerebral Perfusion Pressure (CPP) was excluded in this test because it was calculated directly from ICP as shown in Eq. (3.1) [57].

$$
\mathrm{CPP}=\mathrm{ABP}-\mathrm{ICP}
$$

Equation (3.2) shows the Pearson correlation.

$$
\rho(x, y)=\operatorname{Cov}(X, Y) / \sigma(X) \sigma(Y)
$$

Where $\sigma$ is the standard deviation and Cov is the covariance between $x$ and $y$. This coefficient is meaningful when $x$ and $y$ have a linear correlation which initially was investigated by plotting physiological signals versus ICP in this study. The other assumption to run Pearson correlation was additionally hold: Having intervals in the data. As a result, some significant correlations $(\mathrm{p}<.001)$ were achieved by applying this test. The correlation were found between Heart Rate, Pulse, Arterial Blood Pressure Diastolic (ABP Dias), Respiratory and ABP Mean, ECG segment level III and ECG segment level V with ICP as reported in section 4.3.

\subsection{Feature selection: Pearson correlation, information gain and PCA}

For feature selection, 3 different techniques were experimented.

- Pearson Correlation: The requirement of running Pearson correlation were investigated, and the $p$ values - the statistical significance of a hypothesis test - for features that correlate with ICP were determined and shown in section 4.3 .

- Information gain: To decide on the inclusion criteria of variables from which ICP could be modeled, it is important to examine information gain based on entropy to obtain how much information from each variable could be obtained with respect to the class labels of ICP. 
- PCA: To overcome the problem of multi collinearity, a principal component analysis was conducted to decide on the attributes. PCA working based on the eigenvectors and eigenvalues reported 2 components that could be extracted from the features as shown in section 4.3 .

The question of whether there is actually any correlation between body signals with ICP required the "correlation analysis" to be conducted initially to figure out which signals have bivariate correlation with ICP. Correlation is a method for investigating the relationship between two quantitative variables and Pearson's correlation coefficient is a measure of the strength of the association of the two variables. Initially it was investigated for the linear/ non-linear association between the variables and the linear correlation was observed. The correlation coefficient would not be suitable to calculate if the relationship is not always linear. The closer the scatter of factors is to a straight line, the higher the power of association would be between the variables. Pearson correlation analysis was conducted in this analysis and the significance of the test ( $\mathrm{p}$ values) are investigated. This analysis is done in IBM SPSS version 22 [143].

The next analysis needed to be done to answer the question of "how much information could be extracted from the correlated signals with ICP with respect to the defined class labels of ICP”. This would be to choose the inputs conveying more information and disregard the inputs with very weak information with respect to the class labels. Information gain analyses the reduction in entropy of an input with respect to an output [144]. This study is interested to measure the amount of "change" that each of input signals provides to take ICP to a different state (Normal state / Intracranial hypertension state). The Entropy denotes the Shannon's entropy concept as shown in formula 3-3. This analysis is done by using FSelector and RWekajars libraries in RStudio version 3.4 .3 [145] as shown in (Appendix E).

$$
\text { InfoGain }(\text { Class,Attribute })=\text { Entropy }(\text { Class })-\text { Entropy }(\text { Class } \mid \text { Attribute })
$$




\subsection{Factor analysis}

Factor analysis is a data reduction technique. It takes a big set of variables and determines if the data could be reduced or summarized using a small set of variables and components by looking into sets that have very strong inter-correlations within a set of variables which is challenging to do manually [146]. This is especially problematic when a set of variables are not small and one is interested to look for a pattern inside it. So, factor analysis supports overcoming this problem mathematically. This technique is suitable when the aim is to decrease a large number of correlated variables to a more efficient number, to measure a construct so that there is no redundancy in measuring, but rather measuring all aspects of that construct [146]. There are two main approaches to factor analysis: exploratory and confirmatory [147].

Exploratory factor analysis is used typically in early stages of research in which the aim is to develop the theory or to gather information about relationships among variables [147]. Once the confirmatory analysis is conducted, the aim is to confirm or test specific hypothesis that is developed regarding the fundamental structure of a set of variables [147]. Factor analysis is a generic terminology, which represents a couple of different but related techniques. Two related techniques that fall under the category of factor analysis are principal component analysis (PCA) and standard factor analysis [148].

Despite the remarkable similarities of these techniques, there are some important differences involved. In principal component analysis, the original variables are transformed into a smaller set of variables that have a linear correlation. The investigation, in this case would be to investigate the variance in all the variables [148]. In standard factor analysis, a mathematical model is utilized to estimate the factors. In this method, the shared variance would replace with the total variance [147].

\subsubsection{Factor rotation and interpretation}

Once the number of components is determined, the next step would be to interpret them. The rotated components would not change the underlying outcome and they present a pattern called "loadings" which simplifies the interpretation [149]. Principally, it has shown which variables batch together. There are two main approaches to the rotation which result in either uncorrelated or 
correlated factor solution [150]. Uncorrelated rotations, lead to solutions that tend to be easier to interpret and report; However, they do require the assumption that the underlying constructs or items are independent [150]. On the other hand, the correlated approach permits the factors to be correlated but they are more difficult to interpret.

In this thesis, the correlated approach is implemented since the body signals do have correlation among themselves. This was observed as the result of conducting Pearson correlation at the earlier stage of the research. Even though the investigation was initially conducted to find which of the body signals have bivariate correlation with ICP, it was observed that body signals show correlation between themselves, a problem known as multi-collinearity. A problem which had to be solved before the body signals could be fed as inputs to a predictive model. The technique applied in this study is called "vary max" which attempts to minimize the number of variables that have high loading or high inter-correlation. The PCA analysis is implemented in IBM SPSS version $22[143]$.

\subsection{Principal component analysis}

Component extraction defines the least number of items that could be used to best represent the interrelationships amongst those items [151]. There are various ways to extract this number of components and one of the most common extraction techniques is PCA. PCA determines the new axes to represent data onto a lower dimensional space [151]. It finds the eigenvectors of the covariance matrix of the data and then orders the eigenvectors such that the eigenvector with the largest eigenvalue appears in the first column of the eigenvector matrix and the eigenvector with the smallest eigenvalue appears in the last column [151]. The importance of this function is that one could define how many dimensions are required to reduce the data down to, by keeping the first $\mathrm{N}$ columns of eigenvectors which allows reducing the data down to $\mathrm{N}$ dimensions [151]. 


\subsubsection{Component matrix}

The component matrix shows the unrotated loading of each item for components. SPSS uses the Kaiser criterion that retains all the components with eigenvalues above 1 as the default [152]. In the present thesis, a 2-component-solution capable to explain the majority of variance in the dataset was developed.

\subsection{Proposed solution: supervised methodology}

Having completed the correlated feature selection, the present research focused on the ICP status prediction (normal vs intracranial hypertension status). For this aim, the episodes of "intracranial hypertension" needed to be detected initially. These episodes are defined as the elevation of ICP over $20 \mathrm{mmHg}$ that is continuous for 30 minutes [57] and they acted as a sliding window, which captured the episodes of the correlated physiological signals with ICP. This window is used to capture the start and the end time of the correlated physiological signals with ICP (originating from heart channel and respiration channel) that correspond to the same timing of "Intracranial hypertension state" as per the script in (Appendix B). This step is required to ensure intracranial hypertension episodes are detected.

To be able to build a predictive model which could detect intracranial hypertension state's pattern "before they occur" in the behavior of the signal, it is required to find the samples of normal behavior which are occurring prior to the intracranial hypertension state, so called "Pre-raised episodes". It is ideal to predict the "intracranial hypertension episodes" from "pre-raised episodes of ICP" at the earliest. For this aim, the conducted study, investigated for finding patients having various time intervals of normal ICP pattern ( noted as $t$ ), prior to intracranial hypertension pattern. Where " $\mathrm{t}$ " helps to find the right samples in the correlated features with ICP. This would be to provide samples for the neural network to be able to find the mapping between the two states in the maximum possible time intervals in which "intracranial hypertension state" could be modeled from “pre-raised episodes'. 
The investigation was conducted for finding these episodes in different time intervals. For this aim $t=5, t=10, t=15$ and $t=20$ minutes have been tried as demonstrated in Fig.3.6 and the result showed the maximum achievable time interval, in the available dataset, equals 10 minutes.

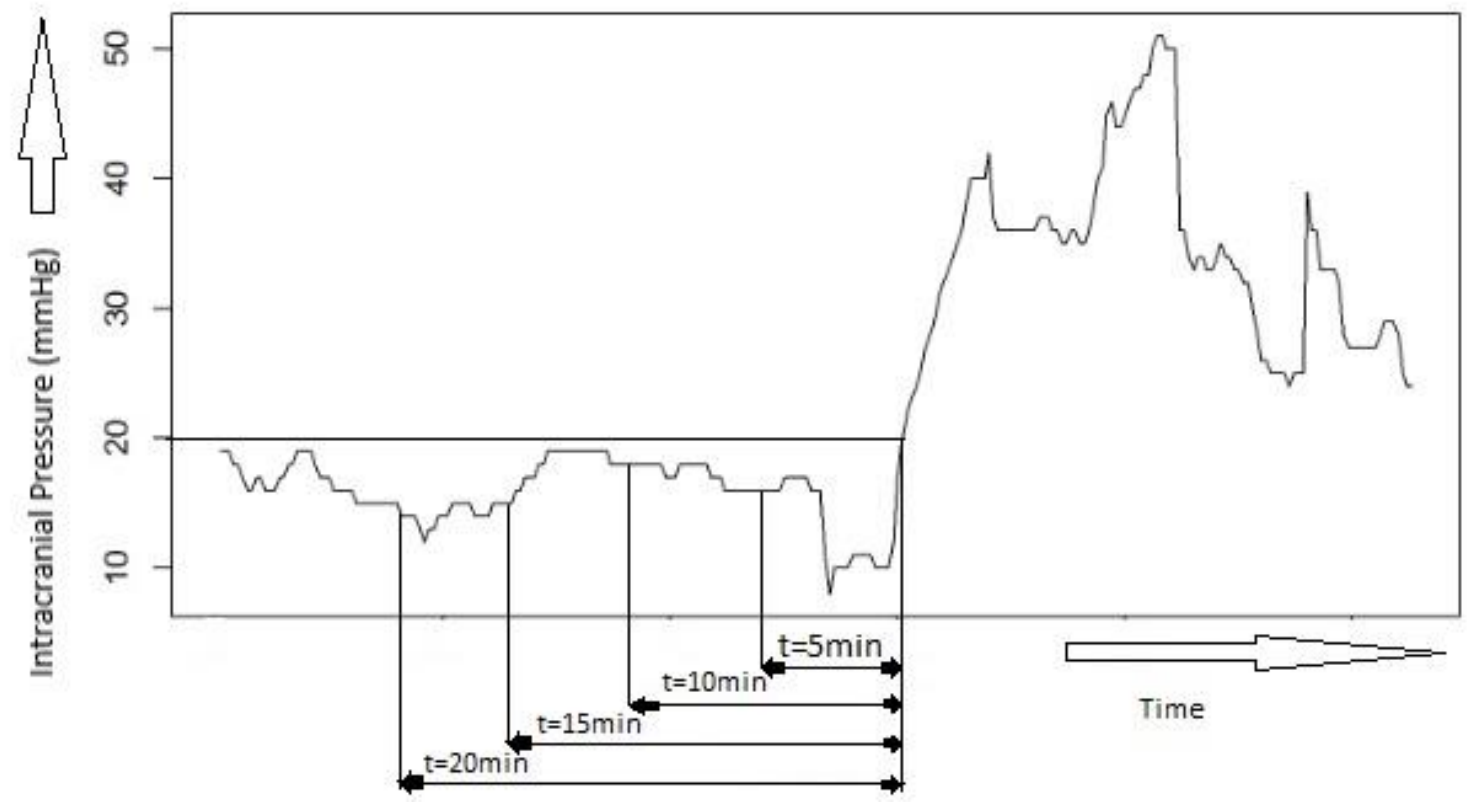

Figure 3.6 Evaluating different time intervals from "pre-raised episodes" to "intracranial hypertension episodes"

This is the maximum available duration of time that sampling was feasible for modeling. The following pseudo code has been proposed and the script is presented in (Appendix F):

1. Make a new column marking whenever ICP $>=20 \mathrm{mmHg}$

2. Detect where ICP markers are continuous either 30 mins forward or 30 mins backwards, if true mark the line numbers in new column valid_high ICP

3. Scan all lines, for each new patient mark first time valid_highICP is TRUE

4. For each patient measure time between history start and first_valid_highICP

5. Select patients with measured time $>=600$ seconds.

Having completed these steps, the correlated body signals with ICP received labels of "Normal" or "Intracranial hypertension". So the time window of "10 minutes normal ICP before 
intracranial hypertension" was detected and the correlated body signals with ICP in that time period received "Intracranial hypertension" label and the rest of the samples received "normal" label.

In the next phase, the PCA analysis is conducted. This step is important to ensure the feature space is containing uncorrelated inputs and the problem of multi-collinearity in feature space is resolved as explained in Sec 3.12. Two components are extracted from the samples and they were fed to the feed forward neural network as inputs. The two PCA components corresponding to 91 patient population $(174,200$ samples) as the input to the feed forward neural network. The output is a binary decision "Intracranial hypertension" versus "normal ICP".

Figure 3.7 demonstrates the proposed methodology for the non-invasive predictive model.

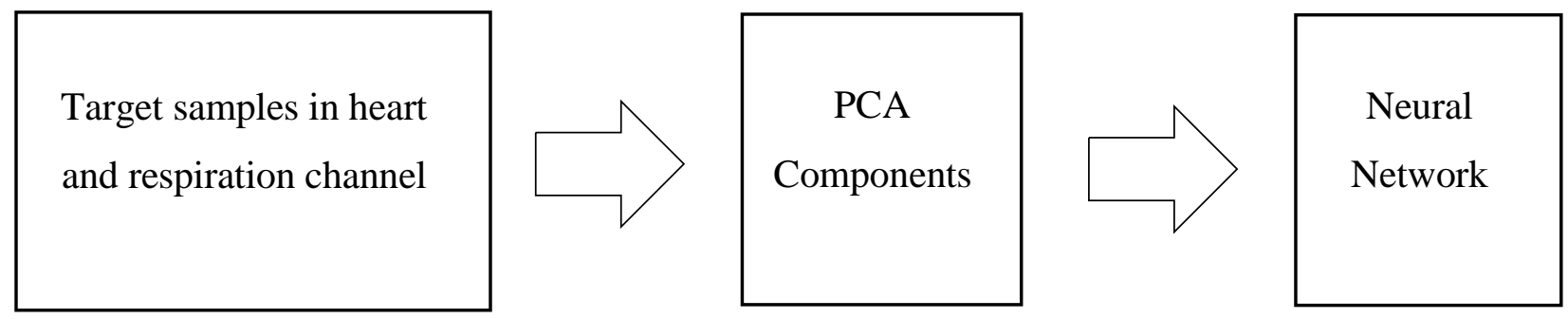

Figure 3.7 Non-invasive predictive methodology diagram

The last phase would be the implementation of a feed forward neural network for predicting the labels of normal ICP vs intracranial hypertension. The input to the neural networks would be two components of PCA and the output would be a binary decision (normal / intracranial hypertension). Neural networks are known for their ability of adaptive learning, real time operation and handling unexpected patterns/inputs [153]. For minimizing the loss function, the stochastic gradient descent is used as the trainer and optimizer of the model. Stochastic Gradient Descent [154] is a learning algorithm used as the optimization method to minimize the loss function with pair $\left(x^{(i)}, y^{(i)}\right)$ from the training examples. In neural networks, this optimizer is often applied with backpropagation that is used for computing gradients by implementing a chain rule of derivatives as shown in the equation 3-4. 


$$
\theta=\theta-\alpha \nabla_{\theta} J\left[\theta ; x^{(i)}, y^{(i)}\right]
$$

Where $\alpha$ is the learning rate and $\theta$ represents the weight. The architecture of the feed forward neural network is demonstrated in Fig 3.8 where the two inputs to the network are component 1 and component 2 obtained from the PCA analysis. The number of hidden layers are two and there are 200 neurons in the first hidden layers and 150 neurons in the second hidden layer.

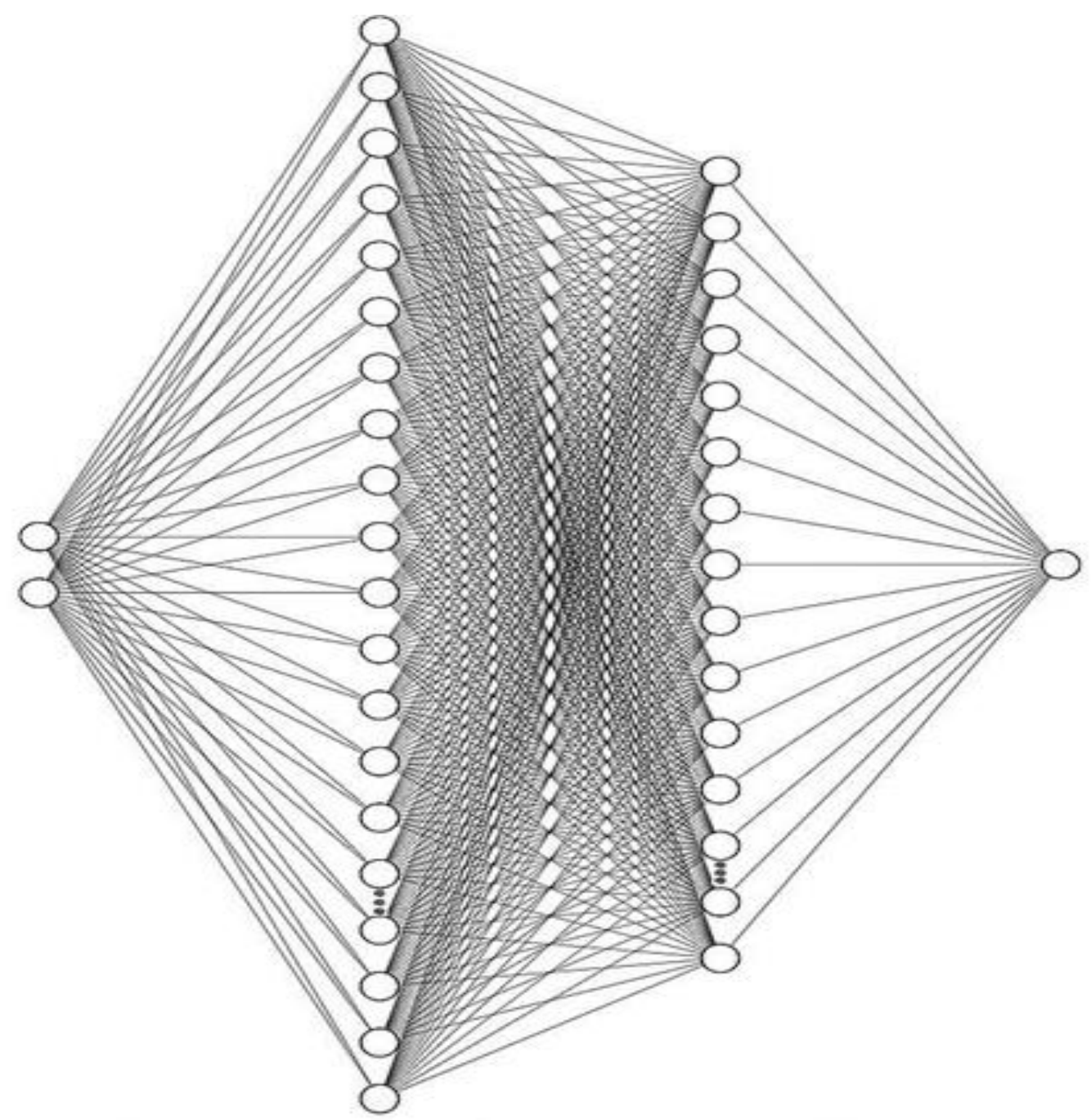

Input layer $\notin R^{2}$

Hidden layer $\notin R^{200}$

Hidden layer $\notin R^{150}$

Output layer $\notin R^{1}$

Figure 3.8 Architecture of the feed forward neural network 


\subsection{Proposed solution: unsupervised methodology}

The hypothesis tested by the unsupervised modeling is that physiological signals could create clusters and show certain behaviors with respect to normal, pre-raised or raised ICP. It is expected that the normal behaviour of ICP form a bigger cluster and the pre-raised and raised form smaller ones. The idea generates from the fact that collecting the members of these clusters i.e. signals from the patients is less risky and the signals are routinely available and if possible, the physiological signals could provide indications of the status of ICP, when invasive monitoring is not available, unfeasible for patients or extra risky.

After showing the correlations of body signals with ICP in table 4.1. The thesis is interested to investigate whether the correlated physiological signals with ICP could create clusters and by analysing the members of the clusters, is it possible to understand changes of ICP? This methodology is designed to answer the last research question of the thesis:

"Is it possible to define a structure from correlating body signals with ICP and interpret those to understand possible status of ICP (normal, pre-raised-raised)?”

The unsupervised methodology should investigate structures through body signals to reply to the aforementioned question and in this thesis, an initial investigation based on the correlation analysis of body signals with ICP was conducted. This modeling should be conducted unsupervised without revealing the existing labels in the dataset to the learning algorithm.

In the next step, samples are transformed with wavelet transformation, which can be shortened or elongated to capture various frequency patterns. As demonstrated in Fig 2.2 in comparison to Fig 2.3, wavelet transformation conveys information of time domain, frequency domain as well as wavelet power levels which reveals a comprehensive picture of the behavior of the signal. Wavelet transformation helps in ensuring that time and frequency of the signals are retained and maximum information of the signals are available. For this aim, wavelets were shifted through the signal along time axis and perform a transformation. For conducting this transformation, the Morelet wavelet has been implemented in $\mathrm{R}$ using WaveletComp library [152]. The input to wavelet transform function are episodes of normal, pre -raised and raised ICP and the output is the wavelet images of these episodes. For this analysis, the number of patient populations is 85 people. 
To conduct the modelling initially $80 \%$ of the labels are disregarded in the dataset and the derived signal from ICP known as cerebral perfusion pressure is also omitted from the samples. $20 \%$ of the samples (labeled data) are kept to be used later for external-validation of the cluster labels as the available ground truth. The $20 \%$ would be the unseen data which are not used in clustering phase but is later used for validating the labels assigned to each cluster. The labels in the unseen data are defined normal for: $5=<\mathrm{ICP}<17$; Pre-raised ICP for $17=<\mathrm{ICP}<20$ and intracranial hypertension for ICP $>=20 \mathrm{mmHg}$ in this modelling.

The $80 \%$ samples (unlabelled data) were transformed to wavelet images and the K-means clustering has been chosen to cluster the sample space into 3 group $(K=3)$ based on the analysis conducted by elbow method (Fig 4.8). Later on, the labels explained in details in sec 4.5 were assigned to each cluster based on the correlation analysis initially conducted in this thesis. Every sample in each cluster got a label at this point.

To evaluate the labels and validating how accurately the methodology works, the last step would be training various classification models with the assigned labels of the clusters and test the classification model on the unseen $(20 \%)$ labels. The conducted experiment revealed if there are learning algorithms that could show high performance on test result.

For this purpose, different classifiers were implemented based on Bernoulli naïve Bayes, Gaussian Naïve Bayes, Logistic regression, Multi-layer perceptron, Multinomial naïve Bayes, Extra tree, Perceptron, Random forest, Stochastic gradient descent's algorithms to evaluate their performance. The result of the experiment is reported in table 4.17 and the best learning algorithms' performance are shown in Fig 4.14 and Fig 4.15. The phases of the unsupervised methodology is shown in Fig 3.9.

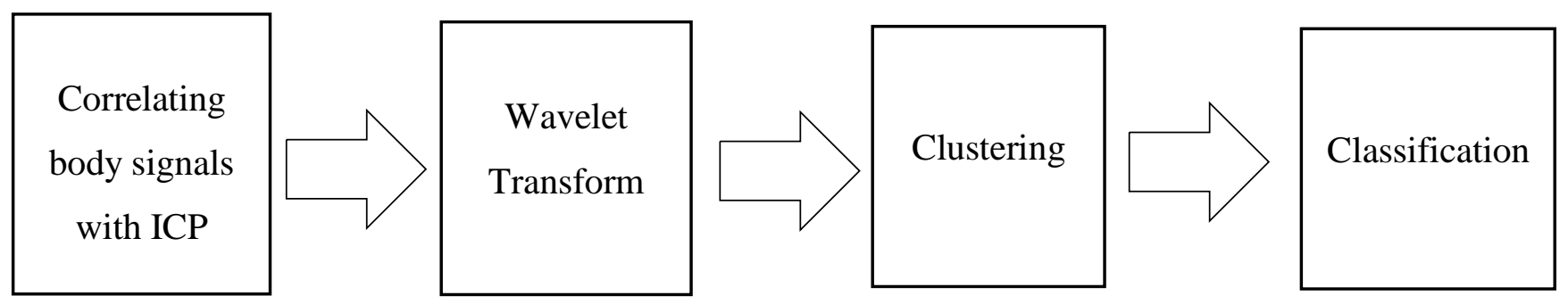

Figure 3.9 Clustering methodology diagram 


\section{Results and Analysis}

\subsection{Introduction}

ICP monitoring is one of the most routinely carried out processes in neuro-critical care units to monitor patients having Traumatic Brain Injuries (TBI). The current medical standard of care using the insertion of a transducer or catheter is primarily based on an invasive technique which usually has certain risks.

The methodology used in this research to address the problem of invasive and reactive detection of raised ICP was to predict the "intracranial hypertension" vs. "normal ICP" through extracted components from correlated body signals with ICP. The solution proposed was based on a feed forward neural network to predict normal vs. intracranial hypertension from body signals mainly through heart and respiration channels. The model is able to predict these states 10 minutes in advance.

On the other hand, the second solution proposed was based on an unsupervised approach in which the most correlated signals with ICP were focused on and it recognized the changes of ICP from the most correlated signals with ICP through clustering. The wavelet clustering was conducted to ultimately lead to non-invasive estimating of ICP status (normal, pre-raised, raised) which was found to be primarily based on the amplitude and frequency modifications of the most correlated body signal with ICP.

Both of the above methodologies became feasible as soon as some significant correlations between ICP and routinely monitored physiological signals in TBI patients were found. The result of the Pearson correlation test indicates that Heart Rate, Pulse, Diastolic Arterial Blood Pressure, Respiration, Mean Arterial Blood Pressure and ECG ST segment levels are significantly correlated with ICP so they have the possibility to be reliable predictors of intracranial hypertension (ICH).

The predictability of these correlating signals with ICP was validated through information gain analysis as well and conducting PCA, the multi-collinearity of the input signals were solved. It was discovered that there was a possible predictive power in the vital signals being routinely monitored from TBI patients. 


\subsection{Result of Pearson correlation analysis and information gain}

The Pearson test demonstrated the bivariate correlations between input and output and also between the variables themselves. This test determined the correlation coefficient between the input (body signals) and the output (ICP) and the effect of other parameters would not be taken into account. The result of the test revealed that there were some significant correlations between several physiological signals and ICP. For interpretation of Pearson correlation results, one must know that the p-value is determined by the observed correlation and the sample size, so with a large enough sample size a very weak correlation could be significant, meaning that what is seen is likely real and not due to chance. On the contrary, with small sample sizes a very strong correlation could be achieved that is not statistically significant.

As demonstrated in table 4.1 it was found that:

- Heart rate and ICP were positively correlated $(\mathrm{R}=0.47, \mathrm{p}<0.001)$

- Pulse and ICP were positively correlated $(\mathrm{R}=0.44, \mathrm{p}<0.001$,

- Respiratory rate and ICP were negatively correlated $(R=-0.78, p<0.001)$

- $\quad$ ST III and ICP were positively correlated $(\mathrm{R}=0.38, \mathrm{p}<0.001)$

- $\mathrm{ABP}$ diastolic and ICP were positively correlated $(\mathrm{R}=0.451, \mathrm{p}<0.001)$

- $\mathrm{ABP}$ mean and ICP were positively correlated $(\mathrm{R}=0.45, \mathrm{p}<0.001)$

- ST V and ICP were negatively correlated $(\mathrm{R}=-0.14, \mathrm{p}<0.001)$

ABP systolic, PVC and SPO2 were proportional with ICP but the correlation was not significant. The Pearson correlation result shows the measure of strength of linear relationship among two variables [155]. 
Table 4.1 The Result of Pearson's Correlation

\begin{tabular}{|c|c|c|c|c|c|c|c|c|c|c|}
\hline \multicolumn{2}{|c|}{ Heart rate } & $\begin{array}{l}\text { Systolic } \\
\text { arterial } \\
\text { blood }\end{array}$ & $\begin{array}{l}\text { Diastolic } \\
\text { blood } \\
\text { pressure }\end{array}$ & Pulse & $\begin{array}{l}\text { Respiration } \\
\text { rate }\end{array}$ & $\begin{array}{c}\text { Mean } \\
\text { arterial } \\
\text { blood } \\
\text { pressure }\end{array}$ & $\begin{array}{c}\text { ECG } \\
\text { Segment } \\
\text { level III }\end{array}$ & $\begin{array}{c}\text { ECG } \\
\text { segme } \\
\text { nt level } \\
\text { V }\end{array}$ & $\mathrm{Sp} 02$ & $\begin{array}{l}\text { Premature } \\
\text { ventricular } \\
\text { contraction }\end{array}$ \\
\hline $\begin{array}{l}\text { Correlation } \\
\text { Coefficients }\end{array}$ & 0.47 & 0.33 & 0.45 & 0.44 & -0.78 & 0.45 & 0.38 & -0.14 & 0.28 & 0.72 \\
\hline Significance & 0.00 & 0.23 & 0.00 & 0.00 & 0.00 & 0.00 & 0.01 & 0.00 & 0.28 & 0.24 \\
\hline R-Squared & 0.22 & 0.11 & 0.20 & 0.20 & 0.62 & 0.20 & 0.15 & -0.02 & 0.08 & 0.52 \\
\hline
\end{tabular}

Although the main focus was to identify the correlations between the inputs -body signalsand the output ICP, some multi-collinearity between the input variables were also detected in this research. Multi-collinearity decreases the learning rate of any machine-learning algorithm. To investigate and measure the amount of the information that each correlated body signal could provide with respect to ICP status, the information gain analysis was performed in the present research and the inter-collinearity problem was solved by conducting a PCA analysis.

The findings are novel in terms of deeper investigation of different leads of heart and respiration system (such as ECG Segment level III and level V) which were not investigated in the literate before and excluding cerebral signals from the feature space in the modeling. Artificial neutral networks as the state of the art could learn the mapping from the heart channel and respiration channel to cerebral channel.

Table 4.2 demonstrates the result of information gain achieved from each physiological attributes and table 4.3 demonstrates information gain from clinical attributes. The measure is interpreted as the information of the class which could be given by the variable. The more the information, the higher possibility to include that variable in modeling. The result of information gain from physiological signals in table 4.2 could have a possible range between $(0, \log 7 \approx 0.845)$. 
Based on the result shown in the table diastolic arterial blood pressure and mean arterial blood pressure provide the highest information with respect to normal vs. intracranial hypertension classes and ECG Segment Level V, respiration rate, heart rate, pulse and ECG Segment level III follows the ranking with respect to the amount of the information they can provide. However, the clinical attributes did not present a satisfactory result and they were not included in further analysis of ICP as shown in table 4.3 .

Table 4.2 Information gain analysis and attribute selection with respect to class labels of ICP

\begin{tabular}{|c|c|}
\hline Physiological Attributes & Information Gain \\
\hline Diastolic Arterial Blood Pressure & 0.615 \\
\hline Mean Arterial Blood Pressure & 0.615 \\
\hline ECG Segment Level V & 0.49 \\
\hline Respiration Rate & 0.44 \\
\hline Heart Rate & 0.39 \\
\hline Pulse & 0.32 \\
\hline ECG Segment Level III & 0.30 \\
\hline
\end{tabular}


Table 4.3 Information Gain analysis from clinical attributes with respect to class labels of ICP and

\begin{tabular}{|c|c|}
\hline Clinical Attributes & Information Gain \\
\hline Daily-Fluid-Input & 0.048 \\
\hline Daily-Fluid-Output & 0.048 \\
\hline $\mathrm{TC}$ & 0.036 \\
\hline $\mathrm{SaO} 2$ & 0.031 \\
\hline FiO2 & 0 \\
\hline GCS-Eye & 0 \\
\hline GCS-Motor & 0 \\
\hline GCD-Verbal & 0 \\
\hline
\end{tabular}

Table 4.4 Attributes and Their Descriptions

\begin{tabular}{|c|c|c|c|}
\hline Variables & Description & Type & Range \\
\hline \multirow{2}{*}{ ABP Mean } & $\begin{array}{c}\text { Mean Arterial blood pressure (invasive, } \\
\text { from one of the radial arteries) }\end{array}$ & Time Series & $\begin{array}{c}41-107 \\
\mathrm{mmHg}\end{array}$ \\
\hline \multirow{2}{*}{ RESP } & Uncalibrated respiration waveform. & Time Series & $13-31$ \\
& Number of heartbeats per unit of time. & Time Series & $57-76$ \\
HR & The difference between systolic and & Time Series & BPM \\
\hline \multirow{2}{*}{ Pulse } & diastolic blood pressure. & BPM \\
\hline \multirow{2}{*}{ ABP Diastolic } & Minimum arterial blood pressure. & Time Series & $15-81$ \\
& ECG ST segment level. & Time Series & $-0.1-0.1$ \\
\hline ST III & ECG ST segment level. & Time Series & $0.3-0.5$ \\
\hline ST V & E & & \\
\hline
\end{tabular}




\subsection{Result of principal component analysis}

In this section, the optimum number of principle components are investigated. For this aim, three tests are conducted to determine the efficiency of variance measurements based on:

- Kaiser's Criterion

- Scree test

- Parallel analysis

The 3 tests served as the standard and popular tests for conducting dimensionality assessment operating based on the eigenvalues of the correlation matrix. The eigenvalues of 1.0 or greater were chosen to be included in these analyses.

There were 3 basic steps in the performance of principal component analysis. These steps are based on the assumptions that must be hold to perform principal component analysis [156]. The first step was to assess the suitability of data for factor analysis as shown in table 4.5. There were 2 parameters to be determined if a dataset was found suitable; 1- the sample size, 2- the strength of the relationship among the variables [156].

The second step is the strength of inter correlation among the items. The standard approach to deal with this issue, requires items to have bivariate correlation of at least $r=>0.30$ [156]. On the other hand, in case there were only few correlations above this level, then factor analysis might not be appropriate. There were some statistical measures generated by SPSS in this study to help determine the appropriateness of the inter relationships. Bartlett's test and Kaiser-Meyer-Olkin Measure of Sampling Adequacy (KMO) validated the appropriateness of the data for principal component analysis. To pass the suitability test, the p-value being used by the Bartlett's test should be significant $(\mathrm{p}<0.05)$.

Moreover, The KMO (measure of sampling adequacy) and Bartlet's test as shown in table 4.5 demonstrates two tests that indicate the suitability of the data for structure detection. The KMO is a statistic that specifies the proportion of variance in the variables that could be caused by the underlying factors. High values considered close to 1.0 usually indicate that a factor analysis 
might be beneficial with the data. Values less than 0.50 indicate that the results of the factor analysis possibly are not very useful. The result of 0.69 demonstrates that the pass mark for factor analysis.

Bartlett's test of sphericity conducts assessments on the hypothesis that the correlation matrix is an identity matrix, which would designate that the variables are unrelated and consequently unsuitable for structure detection. Small values considered to be less than 0.05 of the significance level specify that a factor analysis might be beneficial with the data.

The KMO (ranging from 0 to 1 ) needed a minimum value of 0.6 for a good factor analysis. However, values greater than 0.6 are better options while values close to 1 were ideal [157] [158][159]. To verify if the dataset was suitable for factor analysis the KMO was investigated and reported in table 4.5. Where Df represents the degree of freedom and Sig. stands for significance of the test. The table illustrated the KMO measurement being close to 0.7. In this case, the Bartlett's test was also investigated to be significant with a p-value of 0.001 . Based on the results, factor analysis was found to be appropriate for the present data. In the third step which was to determine the required number of factors, a tradeoff between efficiency and completeness in measuring the interested target was required.

Table 4.5 KMO and Bartlett's Test

\begin{tabular}{|c|c|c|}
\hline \multicolumn{3}{|c|}{ KMO and Bartlett's Test } \\
\hline \multicolumn{2}{|c|}{ Kaiser-Meyer-Olkin Measure of Sampling Adequacy. } & 0.695 \\
\hline \multirow{3}{*}{$\begin{array}{c}\text { Bartlett's Test of } \\
\text { Sphericity }\end{array}$} & Approx. Chi-Square & 471.452 \\
\cline { 2 - 3 } & Df & 21 \\
\cline { 2 - 3 } & Sig. & 0.001 \\
\hline
\end{tabular}


In table 4.6, the principle component analysis extraction is demonstrated and it showed the PCA analysis capability to explain that proportion of the variance in each variable. The initial value of communalities in this analysis is 1 by definition.

Table 4.6 Principal Component Extraction

\begin{tabular}{|c|c|c|}
\hline \multicolumn{2}{|c|}{ Communalities } \\
\hline Heart rate & Initial & Extraction \\
\hline Pulse & 1.000 & 0.687 \\
\hline $\begin{array}{c}\text { Diastolic arterial blood } \\
\text { pressure }\end{array}$ & 1.000 & 0.524 \\
\hline $\begin{array}{c}\text { Mean arterial blood } \\
\text { pressure }\end{array}$ & 1.000 & 0.861 \\
\hline Respiration rate & 1.000 & 0.845 \\
\hline ECG Segment level III & 1.000 & 0.625 \\
\hline ECG segment level V & 1.000 & 0.796 \\
\hline \multicolumn{2}{|c|}{ Extraction Method: Principal Component Analysis. } \\
\hline
\end{tabular}

The rotated component table (Pattern matrix) as shown in table 4.7 is the key matrix of interest. The research had interest to notice if the contribution of the seven correlated signals with ICP can be reduced to smaller number of contributing components and consequently conduct dimensionality reduction of the data. The pattern matrix showed that:

- The variance of ECG segment level V, heart rate and pulse are strongly explained by component 1.

- The variance of diastolic arterial blood pressure, mean arterial blood pressure and respiration rate are strongly explained by the variance of component 2.

- $\quad$ ECG Segment level III is explained by both component 1 and component 2.

The rotated four-factor solutions shown in the pattern matrix was optimal for component 1 and 2. This confirmed that the two-component solution is our best option. Components 1 and 2 were the 
new base for expressing "normal ICP/Intracranial hypertension" in term of 2 components, with all the advantages of having a lower number of coefficients for a neural network model has -such as computational costs, speed, etc.- to predict ICP labels.

Table 4.7 Pattern Matrix

\begin{tabular}{|c|c|c|}
\hline \multicolumn{3}{|c|}{ Components } \\
\hline ECG Segment level III & 1 & -0.513 \\
\hline ECG segment level V & 0.816 & 0.871 \\
\hline Heart rate & 0.776 & 0.851 \\
\hline Pulse & 0.695 & 0.801 \\
\hline $\begin{array}{c}\text { Diastolic arterial blood } \\
\text { pressure }\end{array}$ & & \\
\hline Mean arterial blood pressure & & \\
\hline Respiration rate & & \\
\hline \multicolumn{2}{|c|}{ Extraction Method: Principal Component Analysis. } \\
\hline \multicolumn{2}{|c|}{ Rotation converged in 10 iterations. } \\
\hline
\end{tabular}

As shown in table 4.8, the component table provided the result of the PCA before rotation. It demonstrated to extent to which each variable (pulse, ECG segment level V, heart rate, etc) are "loaded on the components". More specifically, it listed the correlation coefficients between physiological body signals and the components. The result shows that:

- Mean arterial blood pressure correlates in the same direction with component 1 (strongly) and component 2 (fairly).

- Diastolic arterial blood pressure correlates in the same direction with component 1 (strongly) and component 2 (fairly).

- Heart rate correlates in the same direction with component 1 (strongly) and in opposite direction with component 2 (fairly). 
- ECG Segment level V correlates in the opposite direction with component 1 (strongly) and in same direction with Component 2 (fairly).

- Pulse correlates in the same direction with component 1 (strongly) and in opposite direction with component 2 (fairly).

- ECG Segment level III correlates in the opposite direction with component 2 (strongly) and it is not correlated with component 1.

- Respiration rate correlates in the same direction with component 1 and component 2 (fairly).

The component matrix table has been configured that loadings which has less than 0.4 to be suppressed in the result, this may case blank spaces for some of the loadings.

Table 4.8 Component Matrix

\begin{tabular}{|c|c|c|}
\hline \multicolumn{2}{|c|}{ Component Matrix } \\
\hline \multirow{2}{*}{$\begin{array}{c}\text { Mean arterial blood } \\
\text { pressure }\end{array}$} & 1 & 2 \\
\cline { 2 - 4 } & 0.819 & 0.418 \\
\hline $\begin{array}{c}\text { Diastolic arterial blood } \\
\text { pressure }\end{array}$ & 0.813 & 0.447 \\
\hline Heart rate & 0.746 & -0.361 \\
\hline ECG segment level V & -0.737 & 0.411 \\
\hline Pulse & 0.630 & -0.357 \\
\hline ECG Segment level III & \multicolumn{2}{|c|}{ Component } \\
\hline Respiration rate & 0.500 & -0.856 \\
\hline \multicolumn{2}{|c|}{ Extraction Method: Principal Component Analysis. } \\
\hline
\end{tabular}


Table 4.9 demonstrated the total variance explained by different number of components. The table demonstrated that $72.1 \%$ of the total variance can be explained by two components. The table also showed that only 2 components have eigenvalues above 1 . This concludes that [160] the two dimensions in the component space explain $72.14 \%$ of the variance in the data.

Table 4.9 Total Variance Explained

\begin{tabular}{|c|c|c|c|c|c|c|c|}
\hline \multicolumn{8}{|c|}{ Total Variance Explained } \\
\hline \multirow{2}{*}{ 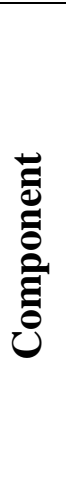 } & \multicolumn{3}{|c|}{ Initial Eigenvalues } & \multicolumn{3}{|c|}{ Extraction Sums of Squared Loadings } & $\begin{array}{c}\text { Rotation } \\
\text { Sums of } \\
\text { Squared } \\
\text { Loadings }\end{array}$ \\
\hline & Total & $\begin{array}{c}\text { Percentage } \\
\text { of } \\
\text { Variance }\end{array}$ & $\begin{array}{l}\text { Cumulative } \\
\text { percentage }\end{array}$ & Total & $\begin{array}{c}\text { Percentage } \\
\text { of } \\
\text { Variance }\end{array}$ & $\begin{array}{l}\text { Cumulative } \\
\text { percentage }\end{array}$ & Total \\
\hline 1 & 3.141 & 44.871 & 44.871 & 3.141 & 44.871 & 44.871 & 2.652 \\
\hline 2 & 1.909 & 27.274 & 72.145 & 1.909 & 27.274 & 72.145 & 2.592 \\
\hline 3 & 0.774 & 11.057 & 83.202 & & & & \\
\hline 4 & 0.486 & 6.947 & 90.149 & & & & \\
\hline 5 & 0.327 & 4.678 & 94.827 & & & & \\
\hline 6 & 0.279 & 3.992 & 98.819 & & & & \\
\hline 7 & 0.083 & 1.181 & 100.000 & & & & \\
\hline
\end{tabular}

Finally, table 4.10 demonstrated the component correlation matrix. 
Table 4.10 Component Correlation Matrix

\begin{tabular}{|cc|c|c|}
\hline Component & 1 & 2 \\
\hline 1 & & 1.000 & 0.158 \\
\hline 2 & 0.158 & 1.000 \\
\hline \multicolumn{3}{|r|}{$\begin{array}{r}\text { Extraction Method: Principal Component Analysis. } \\
\text { Rotation Method: Oblimin with Kaiser Normalization. }\end{array}$} \\
\hline
\end{tabular}

The Scree test is a graphical representation of optimal number of factors to retain and is shown in Fig 4.1. It involved plotting each of the features and then spotting the scatterplot to find a point at which the shape of the curve starts to change direction and becomes horizontal.

The scree plot was investigated to detect a change or an elbow in the line graph. Merely components above the break or elbow are retained [161]. Accordingly, Fig 4.1 demonstrated a break after the third component as the rest of the components do not contain an eigenvalue above one. Therefore, compared to the rest of components, component 1 and 2 were found to explain much more of the variance compared to the remaining components. Therefore, from the graph only two components retained. 


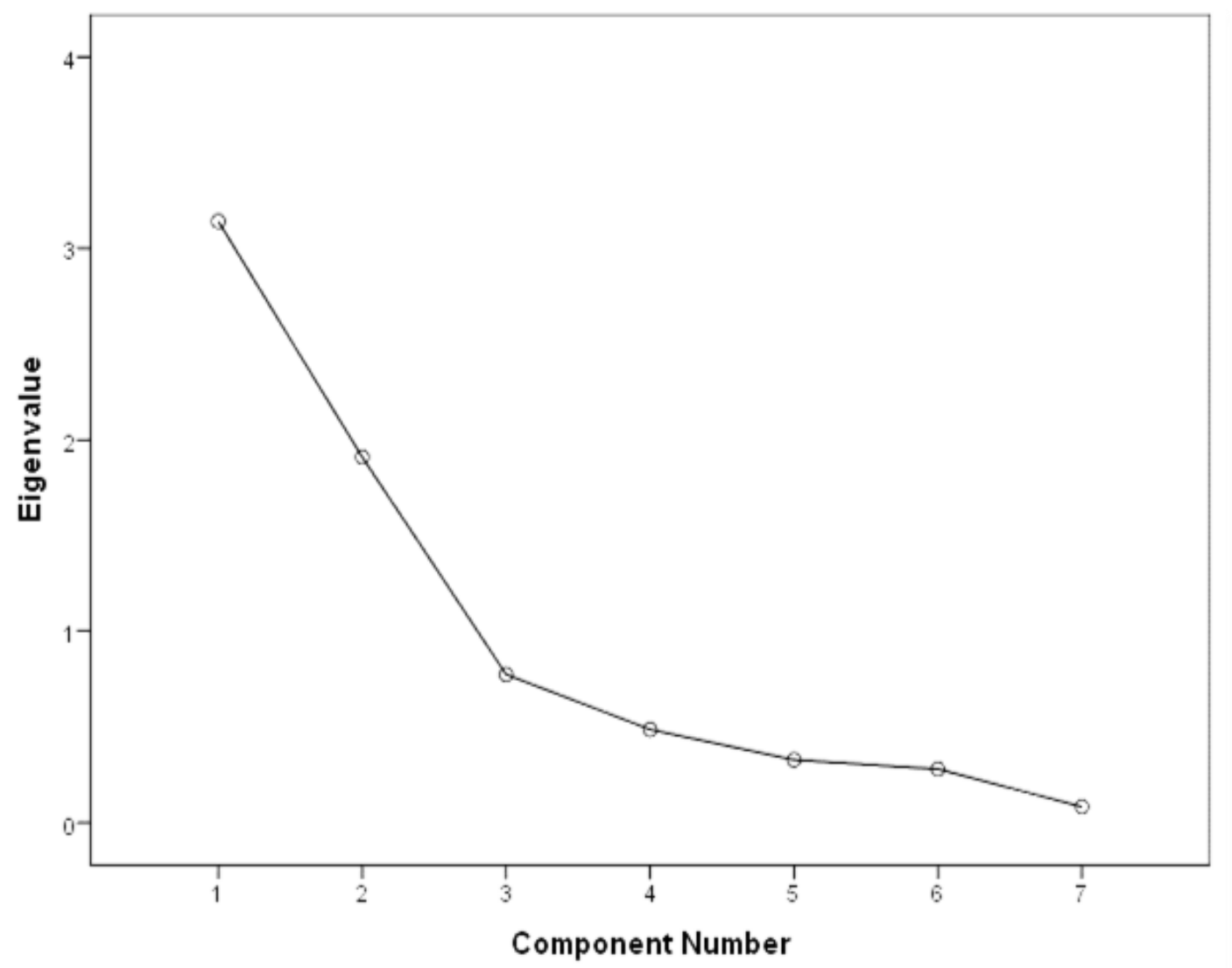

Figure 4.1 Scree Plot

The third method to determine the extraction was the parallel analysis. Monte Carlo PCA [162] was used for Parallel Analysis in the present research. The program contained three variables that need to be defined: number of variable, subjects, and replication which are configured to be 7,120 , and 100 respectively based on the specifications of the samples [163].

Parallel analysis was used as quality control checking as the gold standard by comparing the size of an eigenvalue collected from our own data and then compared to eigenvalues obtained from a randomly generated dataset of the same size and only the eigenvalues that exceeded the randomly generated eigenvalues were retained. It was found that this approach is remarkably more accurate than the Scree test or Kaiser's Criterion, as the latter two methods tend to overestimate the number of components.

Only the components that had an eigenvalue of $=>1$ were extracted. Once there are eigenvalues less than 1.00 , it is not considered to be accurate since they demonstrate less variability as 
compared to a single variable and should not be included for the analysis. Consequently, the result will end up with fewer factor in comparison to the original number of variables [164].

In the conducted test, Monte Carlo PCA generated a random set of eigenvalues that were then used and compared to the eigenvalues of the present data. It generated 100 sets of random data of the same size of the present data and calculated the average eigenvalues from the 100 randomly generated samples. The eigenvalues that were obtained in the dataset are systematically compared with the corresponding first value produced by the Monte Carlo PCA program. All the components with values greater than the criterion value (in the parallel analysis) were retained. After iterating through the eigenvalues within the dataset and comparing them with the parallel analysis, component 1 and 2 were determined to be most influential.

The eigenvalues for components 1 and 2 were found to be greater than the eigenvalues from the parallel analysis. However, the eigenvalues for component 3,4 and 5 were found to be smaller than those of the parallel analysis.

The result of the 3 test were aligned and agreed with each other that the 2 component solution was the optimum number of components and the best solution and they were able to solve the multi-collinearity problem that exists in the physiological signal analysis that is usually neglected in the available signal analysis in the literature. This would also serve to optimize the performance of the neural network and the learning rate was expected to increase for the following reasons:

1. Multi-collinearity is not negligible when conducting analysis on the variables originating from same sources.

2. Multi-collinearity slows down the performance of any learning algorithm when conducted modeling through machine learning techniques.

3. Dimensionality reduction provide by the PCA would serve the learning process and provides the reduces the computational cost. 


\subsection{Result of Prediction of Normal ICP vs. Intracranial Hypertension}

The inputs (correlated body signals with ICP) were selected based on the results of Pearson correlation coefficient test, information gain and the extracted PCA components were fed into an ANN, built with $\mathrm{H} 2 \mathrm{O}$ library [165] in (R), in order to predict the class label of intracranial pressure. The specification of this library and its parameters were shown in table 4.11. The class label prediction varied between Intracranial Hypertension to Normal ICP.

Table 4.11 The configuration of Neural Network

\begin{tabular}{|c|}
\hline H2O library Configuration for Neural Network Implementation \\
\hline standardize $=$ TRUE \\
\hline training_frame $=$ train_h2o \\
\hline validation_frame = test_h2o \\
\hline activation $=$ "Rectifier with dropout" \\
\hline balance_classes $=$ TRUE \\
\hline hidden $=$ c $(50,20)$ \\
\hline nesterov_accelerated_gradient $=\mathrm{T}$ \\
\hline
\end{tabular}

After training various models with different tuning parameters [activation functions, number of hidden neurons and hidden layers, etc.] and comparing the results with respect to minimizing the error, increasing sensitivity, precision, accuracy and specificity and minimizing complexity; the top 5 models were picked. The specificity, accuracy, precision and sensitivity of the 5 best models are defined below and shown in Fig 4.2. Moreover, a sample of confusion matrix is shown in table 4.12 . 
Table 4.12 Sample Confusion Matrix

\begin{tabular}{|c|c|c|c|}
\cline { 3 - 4 } \multicolumn{2}{c|}{} & \multicolumn{2}{c|}{ Predicted } \\
\cline { 3 - 4 } \multicolumn{2}{c|}{} & Negative & Positive \\
\hline \multirow{3}{*}{ Actual } & Negative & A & B \\
\cline { 2 - 4 } & Positive & C & D \\
\hline
\end{tabular}

The metric of accuracy (AC) is the ratio of the correctly predicted data to the total number of data as shown in Formula 4-1 [166]:

$$
A C=\frac{a+d}{a+b+c+d}
$$

The metric of sensitivity so called the true positive rate (TP) is the ratio of positive cases that were correctly identified as shown in formula 4-2 [166]:

$$
T P=\frac{d}{c+d}
$$

The measure of specificity so called the true negative rate (TN) is the proportion of negatives cases that were detected correctly as shown in formula 4-3 [166]:

$$
T N=\frac{a}{a+b}
$$

Finally, the metric of precision $(\mathrm{P})$ is the proportion of the predicted positive cases that were correct. Precision is shown in the formula 4-4 [166]:

$$
P=\frac{d}{b+d}
$$




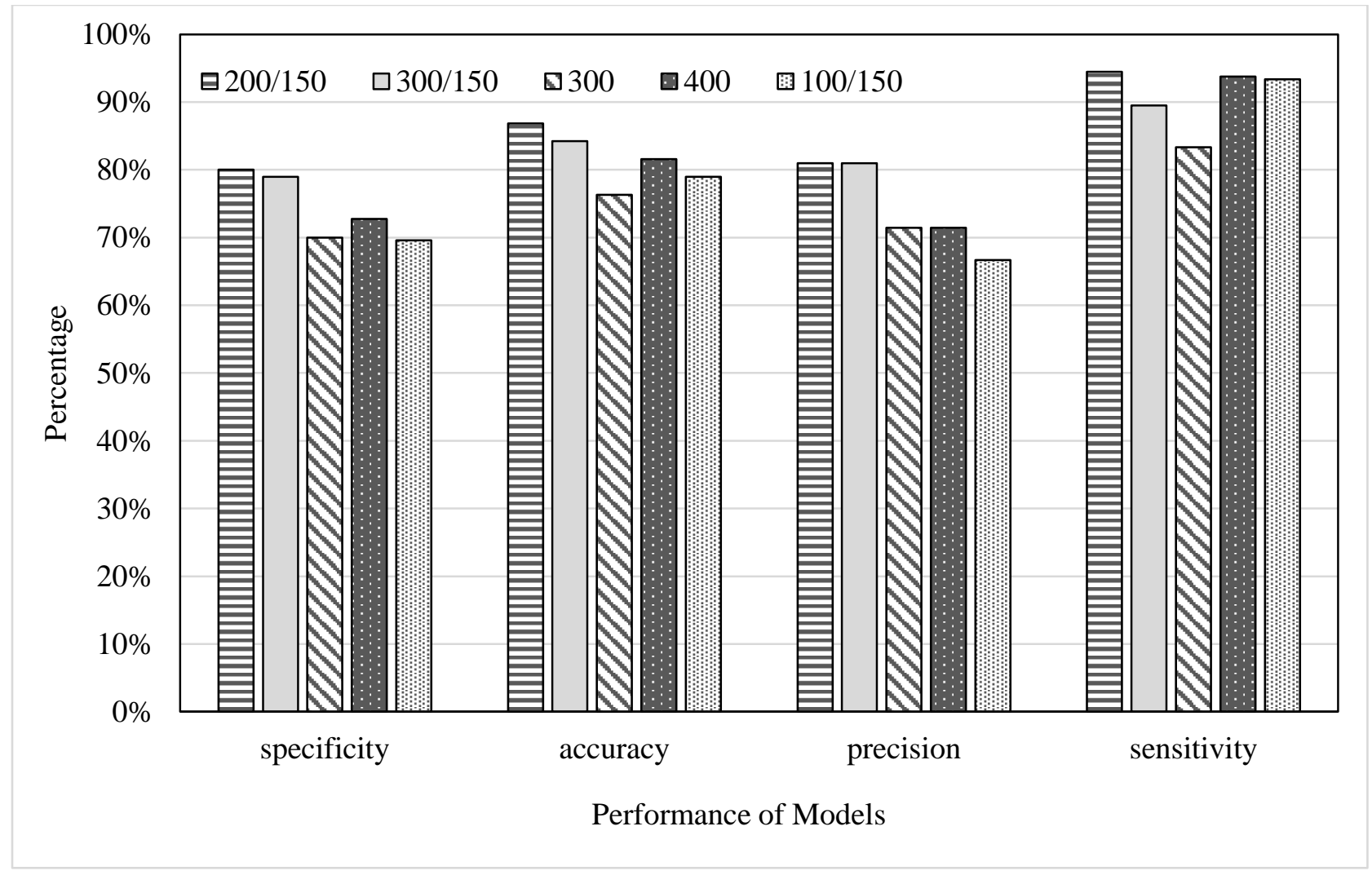

Figure 4.2 Predictive Model Performance with Respect to the Specificity, Accuracy, Precision and Sensitivity

It is important to rely on the performance metrics based on the requirements for the practical applications. As a diagnostic model, the maximum weight on choosing the best model should be on the "sensitivity" metric even at the cost of losing some specificity. An idea diagnostic model should have a high sensitivity and a high specificity, however such models are rarely available and for ICP prediction it has been yet a long journey ahead for all of the proposed models to find their way into clinical practices [167].

In our best 5 models, the model 1 based on 200 neurons layer one and 150 neurons in layer two have similar sensitivity in comparison to model 2 however it has higher specificity, accuracy, and same precision. So we have chosen that one as the best model. Table 4.13 reports the performance of the top 5 models. 
Table 4.13 Models Performance

\begin{tabular}{|c|c|c|c|c|c|}
\hline Experiments & Model 1 & Model 2 & Model 3 & Model 4 & Model 5 \\
\hline $\begin{array}{c}\text { Number of hidden } \\
\text { neurons in each layer }\end{array}$ & $200 / 150$ & $300 / 150$ & 300 & 400 & $100 / 150$ \\
\hline Specificity & $80.0 \%$ & $78.9 \%$ & $70.0 \%$ & $72.7 \%$ & $69.6 \%$ \\
\hline Accuracy & $86.8 \%$ & $84.2 \%$ & $76.3 \%$ & $81.6 \%$ & $78.9 \%$ \\
\hline Precision & $81.0 \%$ & $81.0 \%$ & $71.4 \%$ & $71.4 \%$ & $66.7 \%$ \\
\hline Sensitivity & $94.4 \%$ & $89.5 \%$ & $83.3 \%$ & $93.8 \%$ & $93.3 \%$ \\
\hline
\end{tabular}

Tuning the parameters of ANN structure including the number of hidden layers, type of activation function, the percentage of training and testing data were achieved from trial and error method in order to find the best model that minimizes the error in the ANN. The structure of the neural network was chosen to be Multi-Layer Perceptron (MLP). This feed-forward supervised learning method with stochastic gradient descent optimizer was robust and able to solve complex problems efficiently.

MLP used nonlinear activation functions. Various activation functions such as Tanh, Linear function, Sigmoid function and Rectified Linear (ReLu) were tried and the results obtained using rectified linear unit as activation function of hidden layers and sigmoid as the transfer function of the output layer were found to be the best. Testing different numbers of hidden layers with various numbers of units, the best model was generated from two hidden layers with 200 units in the first hidden layer and 150 units in the second one based on the result of accuracy, sensitivity, specificity and precision of the models and mean squared error as shown in Fig 4.2.

For modeling, $70.3 \%$ of the dataset were used as train set and $29.7 \%$ as test set. Input and output variables were standardized to reduce the likelihood of the local minima problem and improve the training rate resulting in improving the performance of ANN.

The neural network was validated with a 10-fold cross validation method. Fig 4.3-4.7 demonstrated the false positive rate vs true positive rate of the neural network model based on 
different number of neurons in the two hidden layers. The script written for this implementation is demonstrated in (Appendix C).

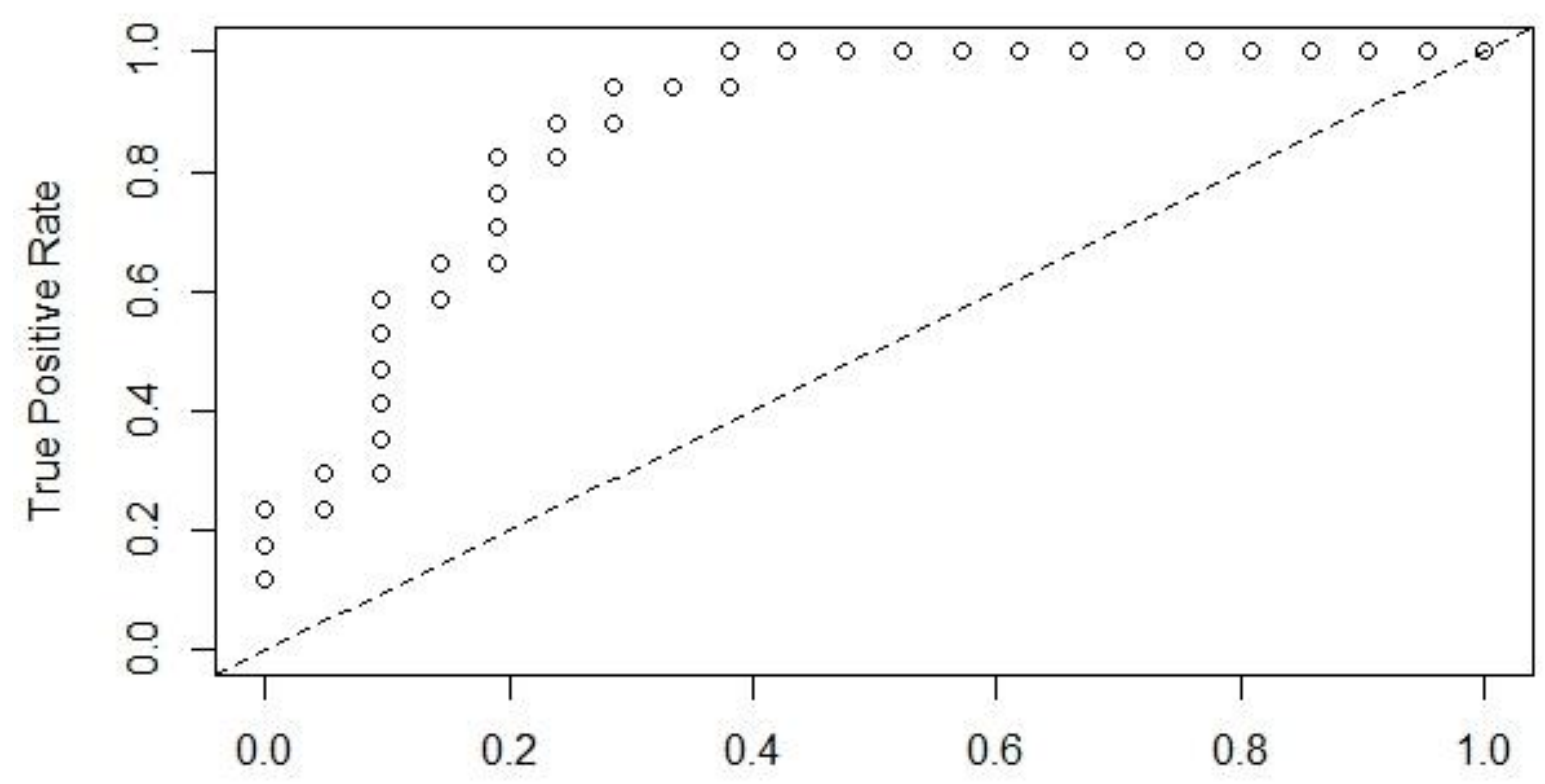

False Positive Rate

Figure 4.3 False Positive Rate vs. True Positive Rate (model 300-150)

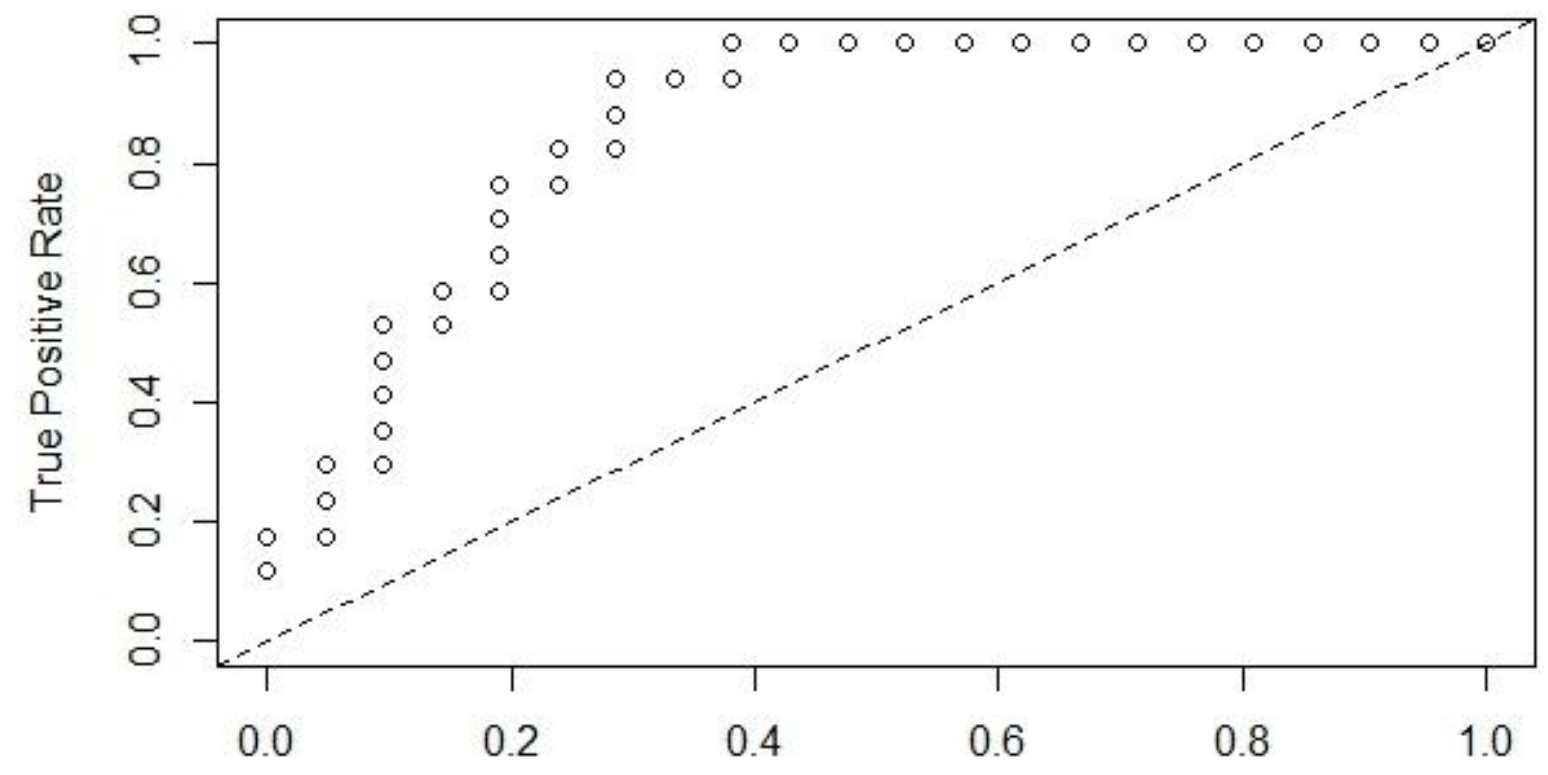

False Positive Rate

Figure 4.4 False Positive Rate vs. True Positive Rate (model 400-400) 


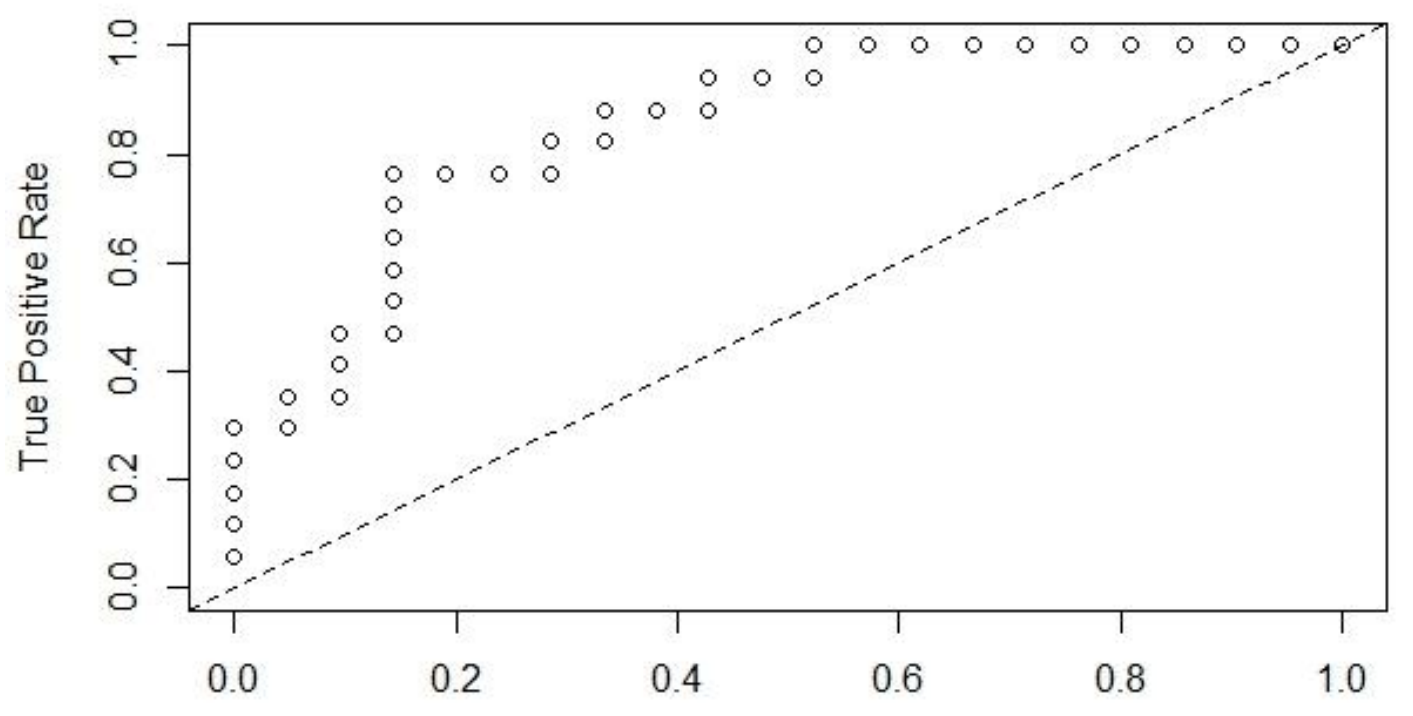

False Positive Rate

Figure 4.5 False Positive Rate vs. True Positive Rate (model 100-150)

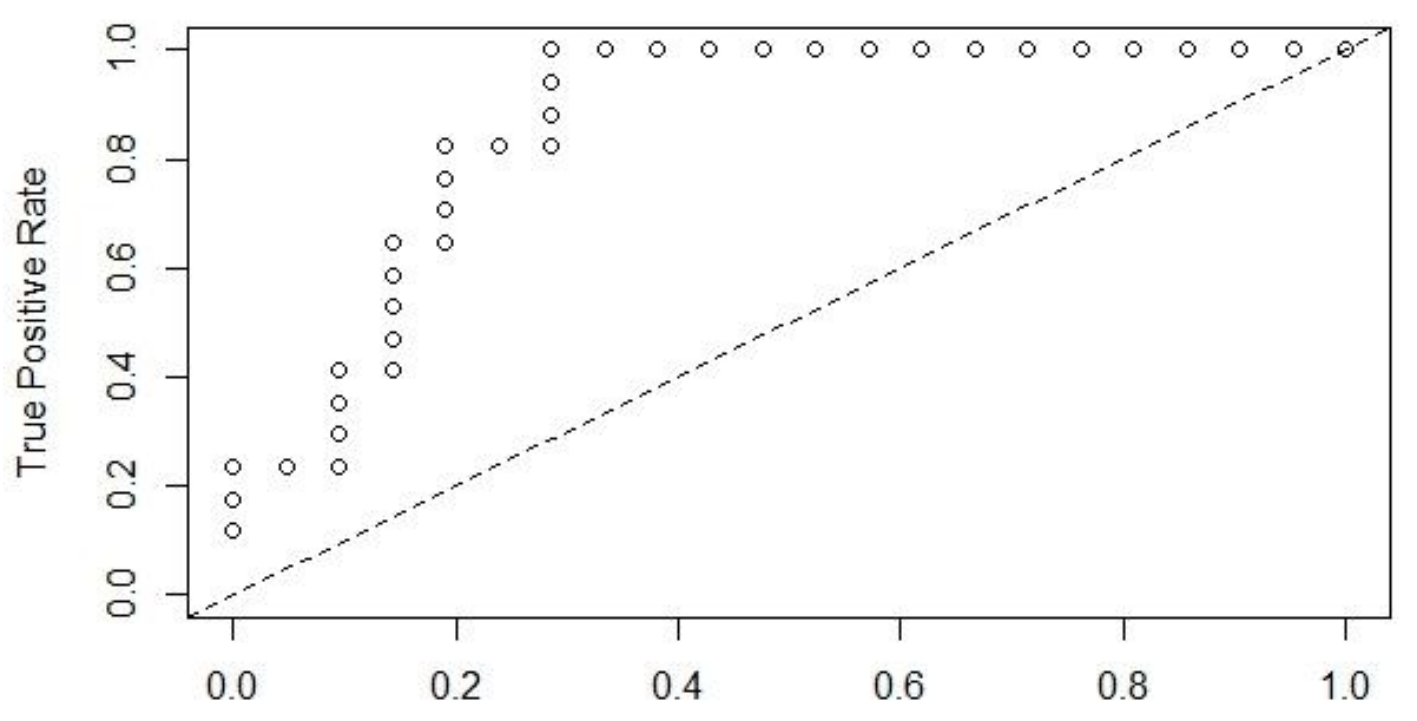

False Positive Rate

Figure 4.6 False Positive Rate vs. True Positive Rate (model 200-150) 


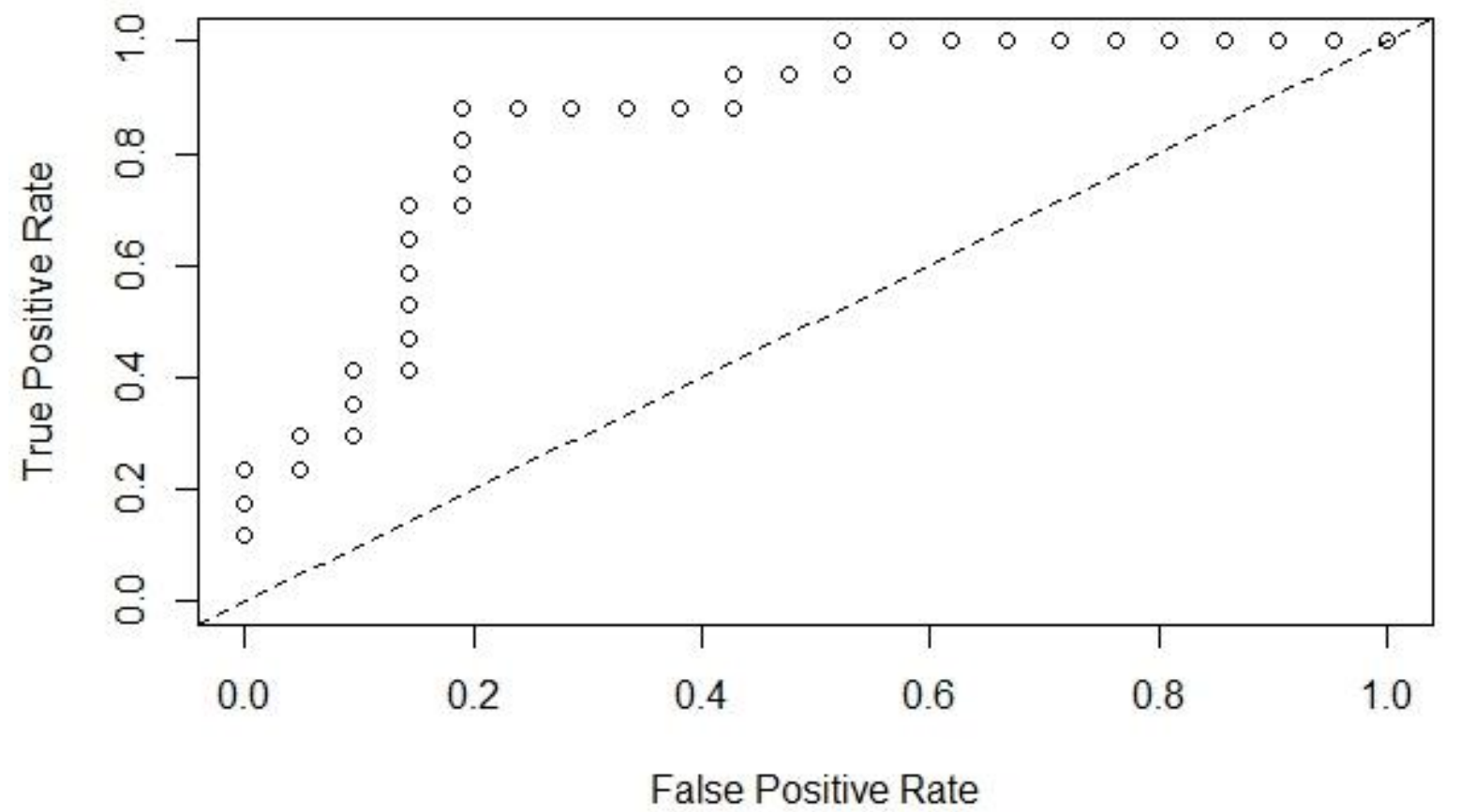

Figure 4.7 False Positive Rate vs. True Positive Rate (model 300-300)

When tested through BrainIT dataset for the generalization purposes, the model confirmed its generalization with quite similar performance. The model showed the mean squared error equal to $0.0536 \mathrm{mmHg}$ and with and the area under the curve (AUROC $=0.94$ ). The generalization condition was tested versus 34727 total samples. The confusion matrix is demonstrated in (Appendix K).

\subsection{Result of Clustering Physiological Signals}

Clustering defined as the partitioning of similar data points into groups or subclasses was done in this research base on similarities of the waveforms. Two well-known applications of K-means clustering are pattern recognition and image processing. The implementation of K-means is explained below: 
1. Placed K points into the space represented by wavelets that were clustered. These points represented initial group centroids.

2. Assigned each wavelets to the group that was closer to the centroid.

3. As soon as all wavelets were assigned, calculated the positions of the $\mathrm{K}$ centroids again.

4. Followed the above process of grouping until the centroids no longer moved which produced a separation of the wavelets into groups from which the target metric for minimization could be calculated.

Considering that the K-means method depended on the $\mathrm{K}$ value, it was crucial to determine its value. The elbow method was used to determine the optimum number of $\mathrm{K}$ value. The Elbow method is a method of validation and interpretation of reliability within cluster analysis designed to support finding the suitable number of clusters in a dataset [168].

Fig 4.8 demonstrated that 3 clusters could reduce the error remarkably; however greater number of clusters does not change the error significantly and hence $\mathrm{K}$ was set as 3 .

Elbow Method Curve

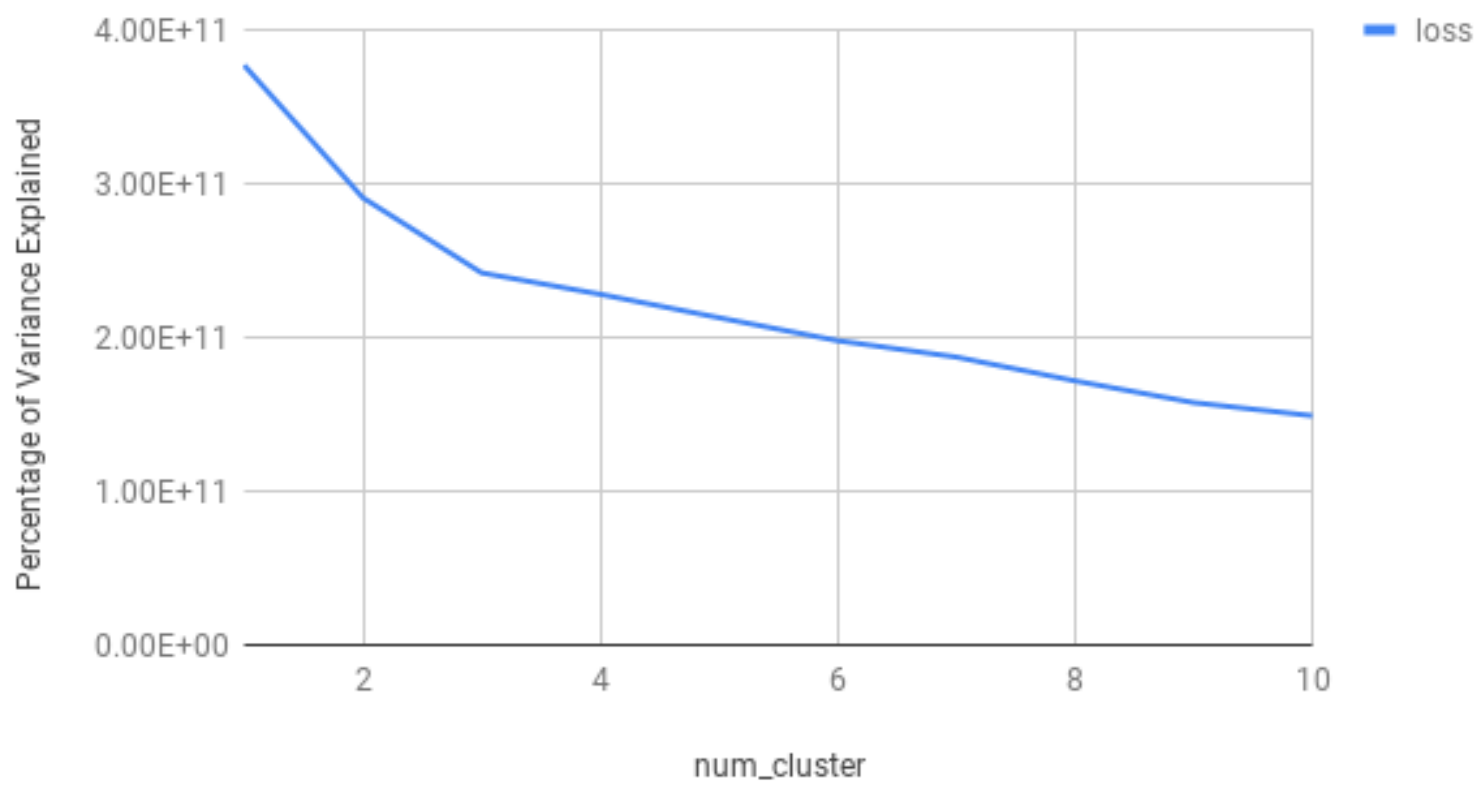

Figure 4.8. Elbow Method to Find the Optimum Number of K 
The clusters were labeled as follows:

- Cluster 0: Labeled as normal ICP. Most of the patients were in normal condition as the heart rate (HR) was within the normal range $(60-100 \mathrm{bpm})$ and the respiratory rate of the patient was also in normal range (12-30/min), these samples are labeled normal. They are defined as normal ICP.

- Cluster 1: Labeled as pre-raised ICP. In most cases the HR was within normal range but there was an increase in the ABP values indicating that the patients could potentially be in ICP pre-raised condition. Due to certain rise in the heart rate and increase in the respiratory rate of the patient, it is concluded that the patients are in pre-raised ICP condition [169] knowing that the increase of arterial blood pressure (mean, systolic and diastolic pressure) and respiration rate, is accompanied by the moderate rise in ICP and ICP could be in its high threshold [170].

- Cluster 2: Labeled as Intracranial Hypertension. It was analyzed that as the respiration rate started to decrease abruptly, ICP could be in its very raised value [171].

Tables 4.14 - 4.16 showed the patients' condition recorded for cluster 1-3, respectively.

\subsection{Statistical report on the clustering result}

Statistical summary was defined as the information such as mean, median, mode, minimum value, maximum value, range, standard deviation etc. that gives insight about the wavelet data sample categorized into each cluster to provide an insight on which ranges of signals have become members of each cluster. 
Table 4.14 Patients' Condition Corresponding to Cluster 0 (Gained the normal label: 48 members)

\begin{tabular}{|c|c|c|c|c|c|c|c|}
\hline $\begin{array}{c}\text { Biomedical } \\
\text { Signals }\end{array}$ & Minimum & Maximum & Mean & Median & Mode & $\begin{array}{c}\text { Std. } \\
\text { Deviation }\end{array}$ & Variance \\
\hline HR & 65 & 76 & 71.81 & 70 & 71 & 2.389 & 5.71 \\
\hline Pulse & 60 & 71 & 66.94 & 69 & 70 & 2.137 & 4.57 \\
\hline ABP Dias & 12 & 61 & 52.05 & 53 & 52 & 3.93 & 15.45 \\
\hline ABP Mean & 38 & 76 & 68.65 & 70 & 70 & 6.61 & 43.7 \\
\hline Respiratory & 13 & 30 & 18.01 & 17 & 15 & 3.40 & 11.57 \\
\hline ST III & -0.1 & 0 & -0.02 & 0 & 0 & 0.031 & 0.001 \\
\hline ST V & 0.2 & 0.4 & 0.24 & 0.2 & 0.2 & 0.085 & 0.0073 \\
\hline
\end{tabular}

Table 4.15 Patients' Condition Corresponding to Cluster 1 (Gained the label of pre-raised ICP: 15 members)

\begin{tabular}{|c|c|c|c|c|c|c|c|}
\hline $\begin{array}{c}\text { Biomedical } \\
\text { Signals }\end{array}$ & Minimum & Maximum & Mean & Median & Mode & $\begin{array}{c}\text { Std. } \\
\text { Deviation }\end{array}$ & Variance \\
\hline HR & 55 & 73 & 60.7 & 60 & 55 & 4.48 & 20.09 \\
\hline Pulse & 56 & 71 & 65.31 & 67 & 70 & 4.91 & 24.13 \\
\hline ABP Dias & 34 & 63 & 58.85 & 61 & 62 & 8.73 & 76.38 \\
\hline ABP Mean & 56 & 84 & 64.68 & 61 & 62 & 8.011 & 64.18 \\
\hline Respiratory & 26 & 30 & 27.27 & 27 & 27 & 1.333 & 1.779 \\
\hline ST III & -0.1 & 0 & -0.017 & 0 & 0 & 0.031 & 0.001 \\
\hline ST V & 0.3 & 0.5 & 0.478 & 0.4 & 0.5 & 0.031 & 0.001 \\
\hline
\end{tabular}

Table 4.16 Patients' Condition Corresponding to cluster 2 (gained the label of intracranial hypertension: 21 members)

\begin{tabular}{|c|c|c|c|c|c|c|c|}
\hline $\begin{array}{c}\text { Biomedical } \\
\text { Signals }\end{array}$ & Minimum & Maximum & Mean & Median & Mode & $\begin{array}{c}\text { Std. } \\
\text { Deviation }\end{array}$ & Variance \\
\hline HR & 67 & 75 & 72.17 & 72 & 71 & 1.467 & 2.155 \\
\hline Pulse & 67 & 71 & 71.36 & 73 & 69 & 1.135 & 1.29 \\
\hline ABP Dias & 43 & 80 & 72.51 & 74 & 74 & 6.238 & 38.92 \\
\hline ABP Mean & 90 & 102 & 93.16 & 98 & 95 & 3.937 & 15.5 \\
\hline Respiratory & 14 & 17 & 15.73 & 19 & 14 & 0.326 & 0.106 \\
\hline ST III & 0 & 0.1 & 0.011 & 0 & 0 & 0.01 & 0.0001 \\
\hline ST V & 0.5 & 0.7 & 0.05845 & 0.2 & 0.5 & 0.063 & 0.004 \\
\hline
\end{tabular}




\subsection{Evaluation of Clustering Labels with Classification}

The clustering result were evaluated by classification on the $20 \%$ unseen data with available labels. As demonstrated in Fig 4.9. It was noticed that the accuracy of the perceptron and multinomial logistic regression are the two highest among all.

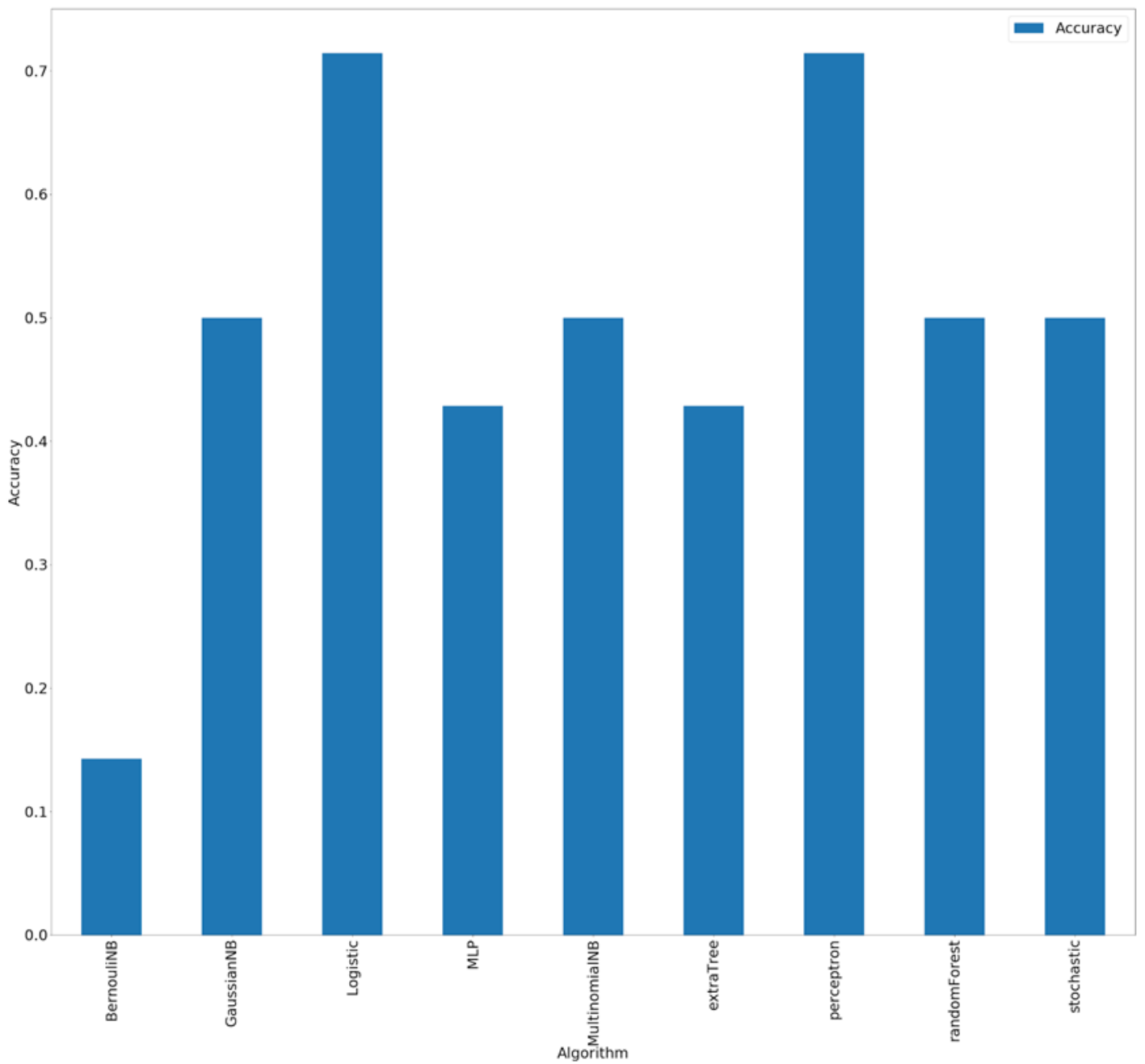

Figure 4.9 the Accuracy of Classification of the Clustering Result 
Figure 4.10 demonstrated sensitivity of different classes 0,1 and 2 as labeled normal, pre-raised and raised. It is observed that the sensitivity of multinomial logistic regression and Perceptron are approximately the same and sensitivity is well distributed among three different classes.

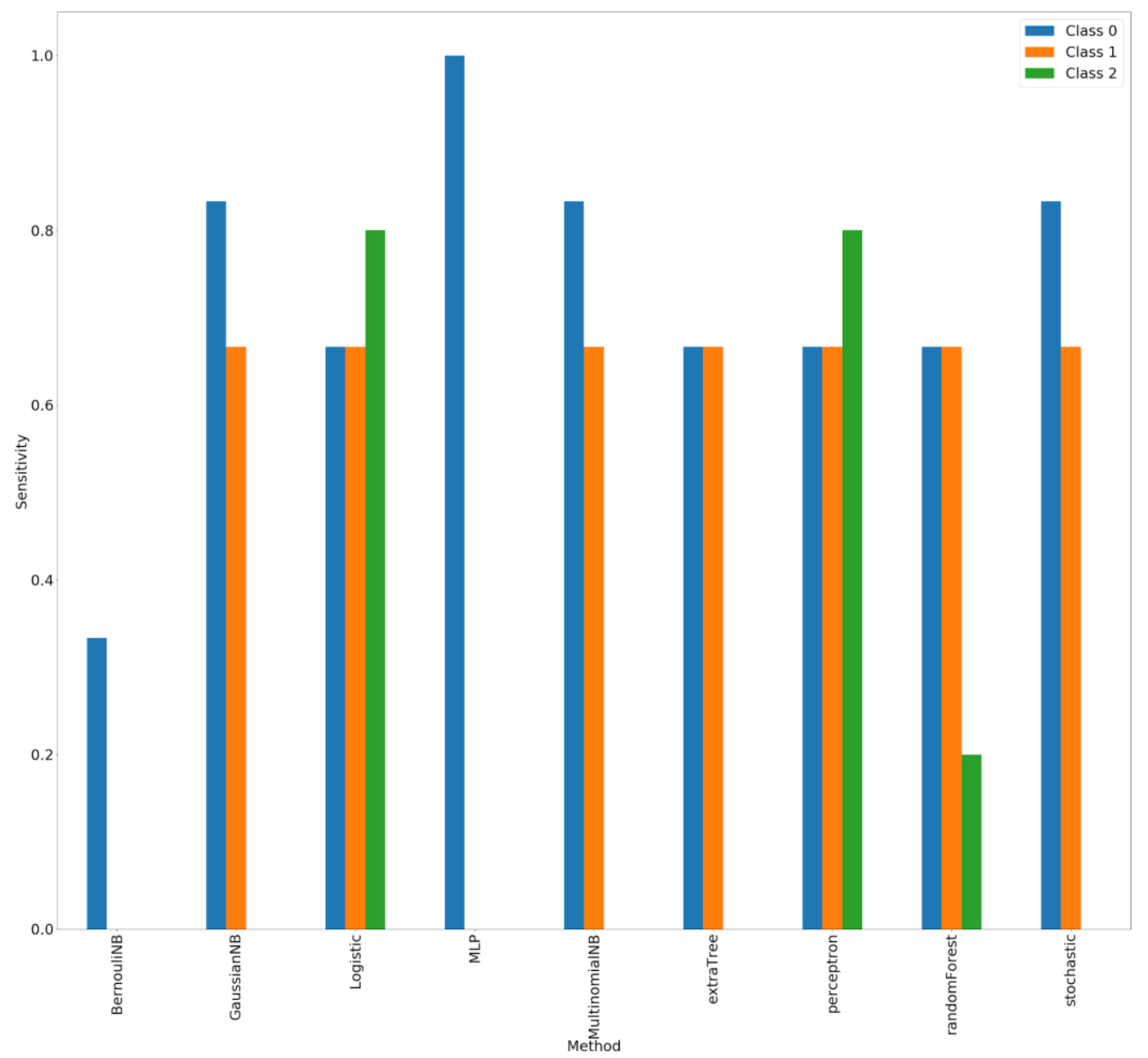

Figure 4.10 Sensitivity of Classification of the Clustering Result

Figure 4.11 demonstrated of the comparison between the precision of different classes for various algorithms which showed that the precision of multinomial logistic regression and perceptron classifier were greater. Precision would be the answer to "what percentage of prediction of the desired label is correct" in this thesis. 


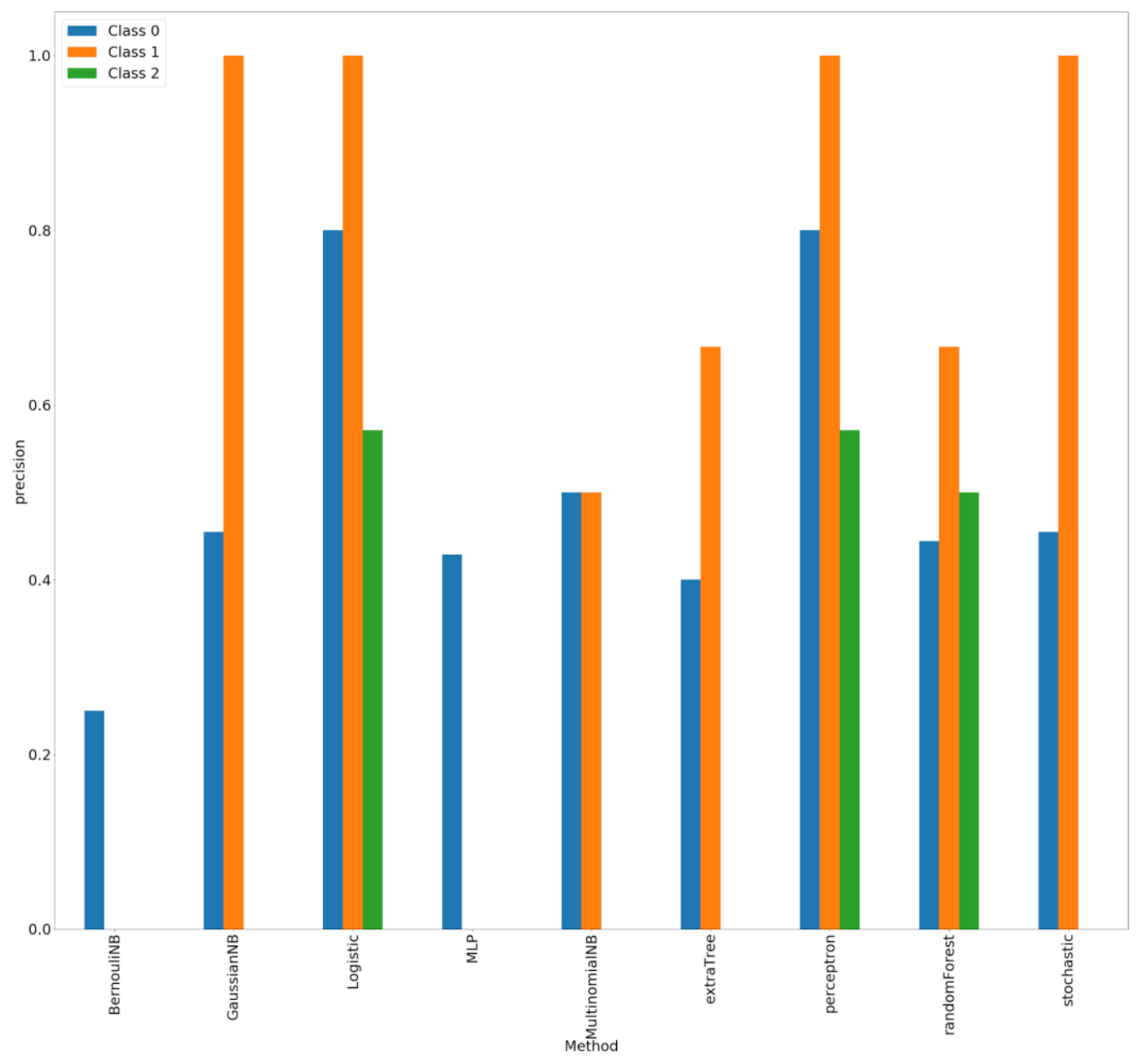

Figure 4.11 Precision of Classification of the Clustering Result

Specificity of various classifier for the three different classes is shown in Fig 4.12. Specificity was defined as a measure of how good the algorithm performed in detecting values that do not belong to a class. The mean squared errors of the algorithms was demonstrated in Fig 4.13. 


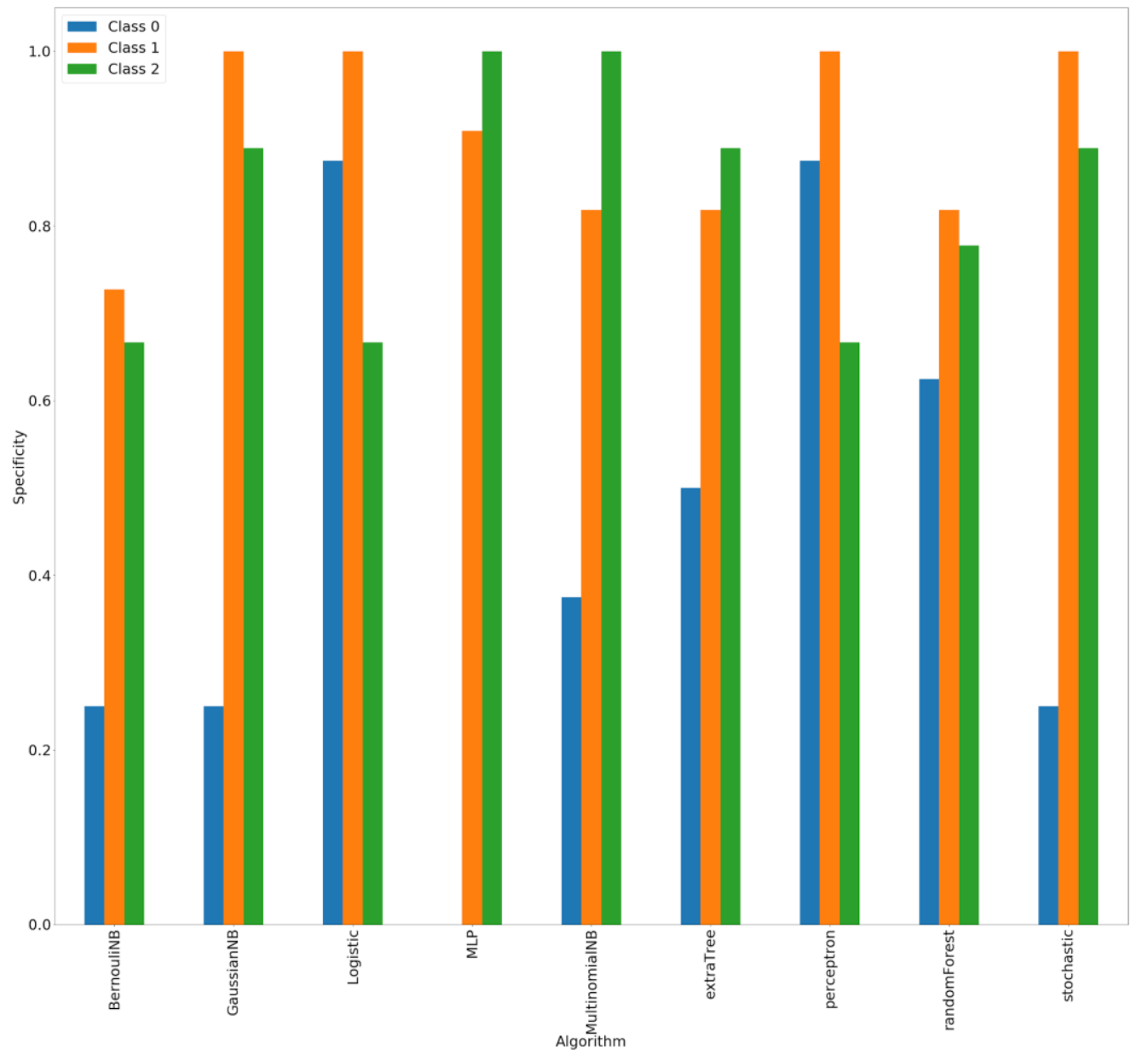

Figure 4.12 Specificity of Classification of the Clustering Result 


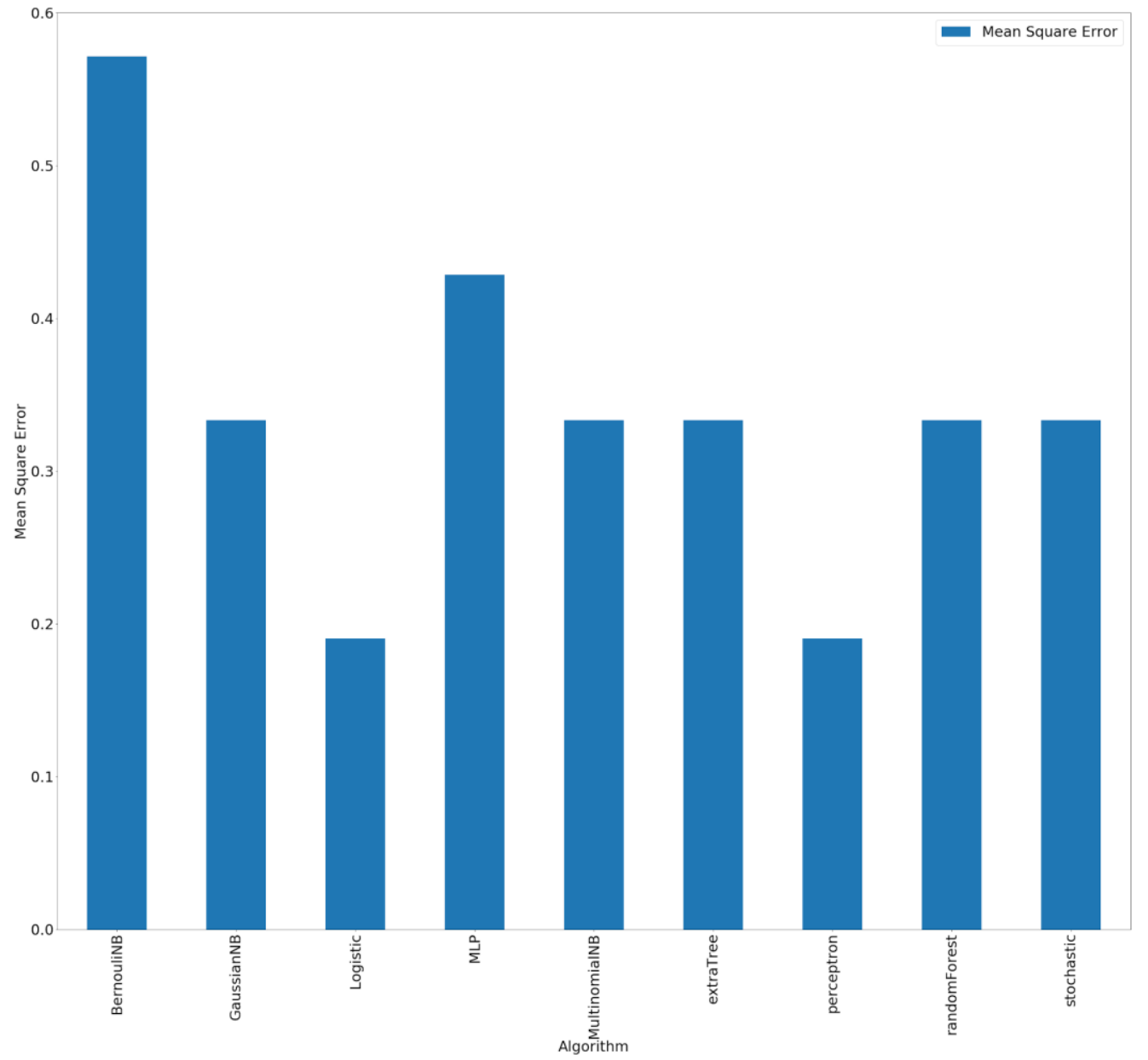

Figure 4.13 Mean Squared Error of Classification of the Clustering Result 
Table 4.17 the Evaluation of the Implemented Classifiers on the Labels

\begin{tabular}{|c|c|c|c|c|c|c|c|c|c|}
\hline 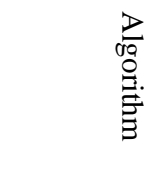 & 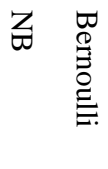 & 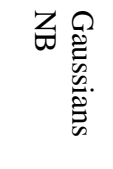 & $\begin{array}{l}5 \\
0 \\
09 . \\
0.0 \\
0 .\end{array}$ & $\underset{\sigma}{3}$ & Z 忐 & 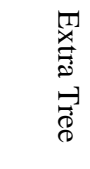 & 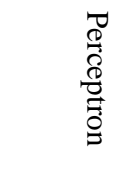 & 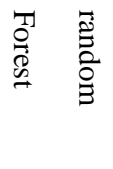 & 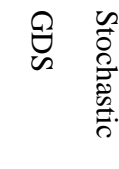 \\
\hline Accuracy & 14.28 & 50 & 71.4 & 42.8 & 59 & 42.8 & 71.4 & 50 & 50 \\
\hline MSE & 0.57 & 0.33 & 0.19 & 0.43 & 0.33 & 0.38 & 0.19 & 0.33 & 0.33 \\
\hline $\begin{array}{c}\text { Sensitivity } \\
\text { Class } 0\end{array}$ & 33.3 & 83.3 & 66.6 & 100 & 83.33 & 66.6 & 66.6 & 66.6 & 83.33 \\
\hline $\begin{array}{c}\text { Sensitivity } \\
\text { Class } 1\end{array}$ & 0 & 66.6 & 66.6 & 0 & 66.6 & 66.6 & 66.6 & 66.6 & 66.6 \\
\hline $\begin{array}{l}\text { Sensitivity } \\
\text { Class } 2\end{array}$ & 0 & 0 & 80 & 0 & 0 & 0 & 80 & 20 & 0 \\
\hline $\begin{array}{c}\text { Specificity } \\
\text { Class } 0\end{array}$ & 25 & 25 & 87.5 & 0 & 37.5 & 25 & 87.5 & 37.5 & 25 \\
\hline $\begin{array}{c}\text { Specificity } \\
\text { Class } 1\end{array}$ & 72.7 & 100 & 100 & 100 & 81.81 & 90.9 & 100 & 90.9 & 100 \\
\hline $\begin{array}{c}\text { Specificity } \\
\text { class } 2\end{array}$ & 72.7 & 88.8 & 66.6 & 100 & 100 & 88.8 & 66.6 & 88.88 & 88.8 \\
\hline $\begin{array}{l}\text { Precision } \\
\text { Class } 0\end{array}$ & 25 & 45.45 & 80 & 42.8 & 50 & 40 & 80 & 44.4 & 45.45 \\
\hline $\begin{array}{l}\text { Precision } \\
\text { Class } 1\end{array}$ & 0 & 100 & 1 & 0 & 50 & 66.6 & 100 & 66.6 & 100 \\
\hline $\begin{array}{l}\text { Precision } \\
\text { Class } 2\end{array}$ & 0 & 0 & 57.14 & 0 & 0 & 0 & 57.1 & 50 & 0 \\
\hline
\end{tabular}

The ROC curves of the best models - multinomial logistic regression - and Perceptron classifier are demonstrated in Fig 4.14 and 4.15 respectively. 


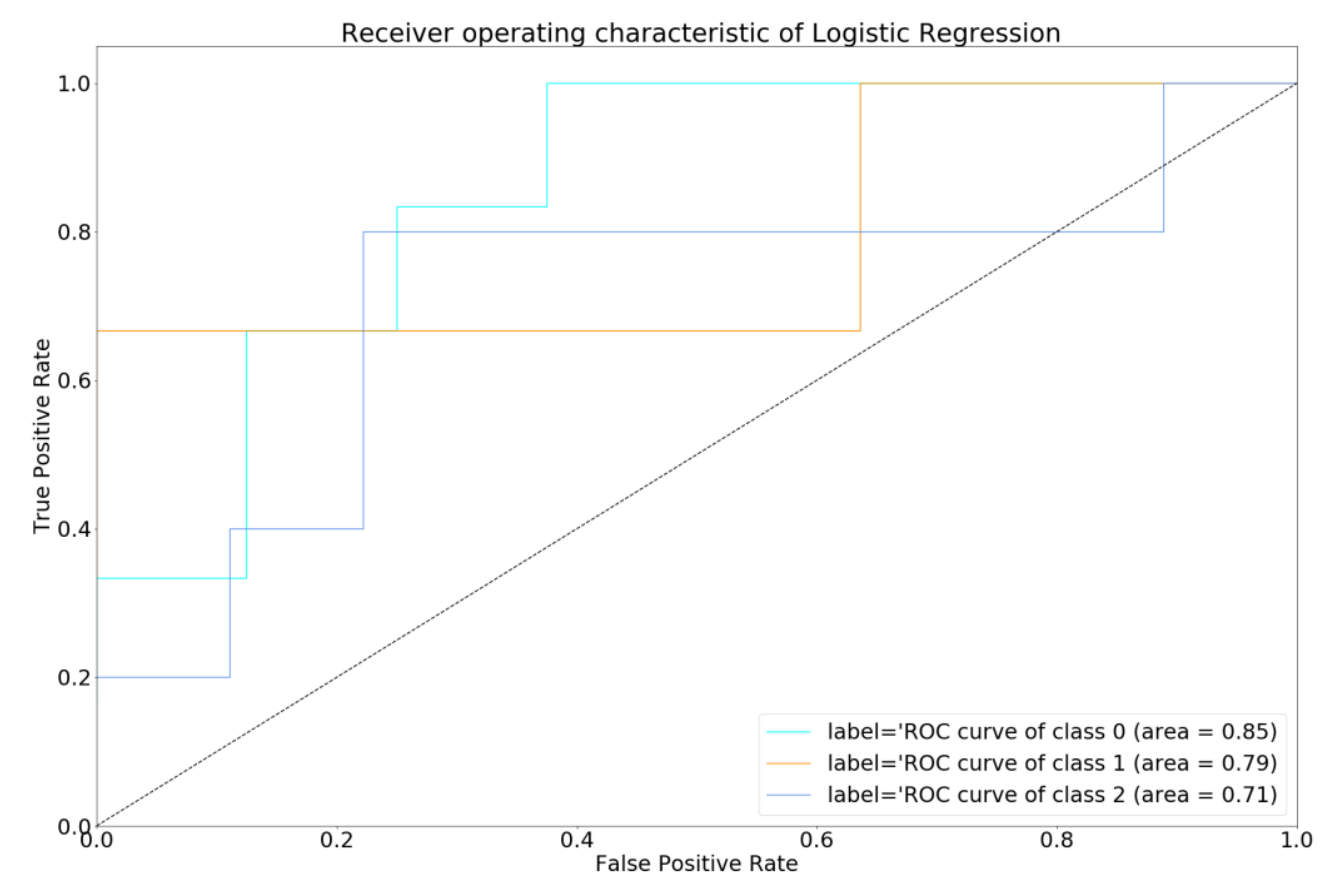

Figure 4.14 ROC of Different Classes Obtained by Multinomial Logistic Regression

In the classification based on clustering result, 3 labels were defined as normal ICP or class 0 , Pre-raised ICP or class 1 and intracranial hypertension or class 2 . The ROC curve generated as shown in Fig 4.14 - Fig 4.15 are using the one vs all strategy, as ROC curve are meant for binary classification. In the 3 ROC curves with each representing the 3 different classes are observed.

The ROC curve is a graph of true positive rate vs false positive rate. The True positive rate answers the question of "how many values belonging to a particular class were identified truly as member of that class". False positive rate answers the question of "whether a particular class was truly identified as a member of a particular class when in fact it was a member of another class."

In Fig 4.14, the graph of true positive rate vs false positive rate of class with labels 0, 1,2 are demonstrated. From the graph it is observed that class 2 has the worst graph since it crosses the 0.5 threshold easily and it could be concluded that the class 2 had the lowest accuracy when it comes to one vs all classification. Also the AUC of class 2 being the lowest proves that its accuracy is lowest when using multinomial logistic regression. ROC curves of class 1 started out promising 
but went dangerously close to 0.5 which meant that there was certain classification wherein the class 1's that were misclassified. ROC curve of class 0 was the best and also had the highest accuracy in all the three classes.

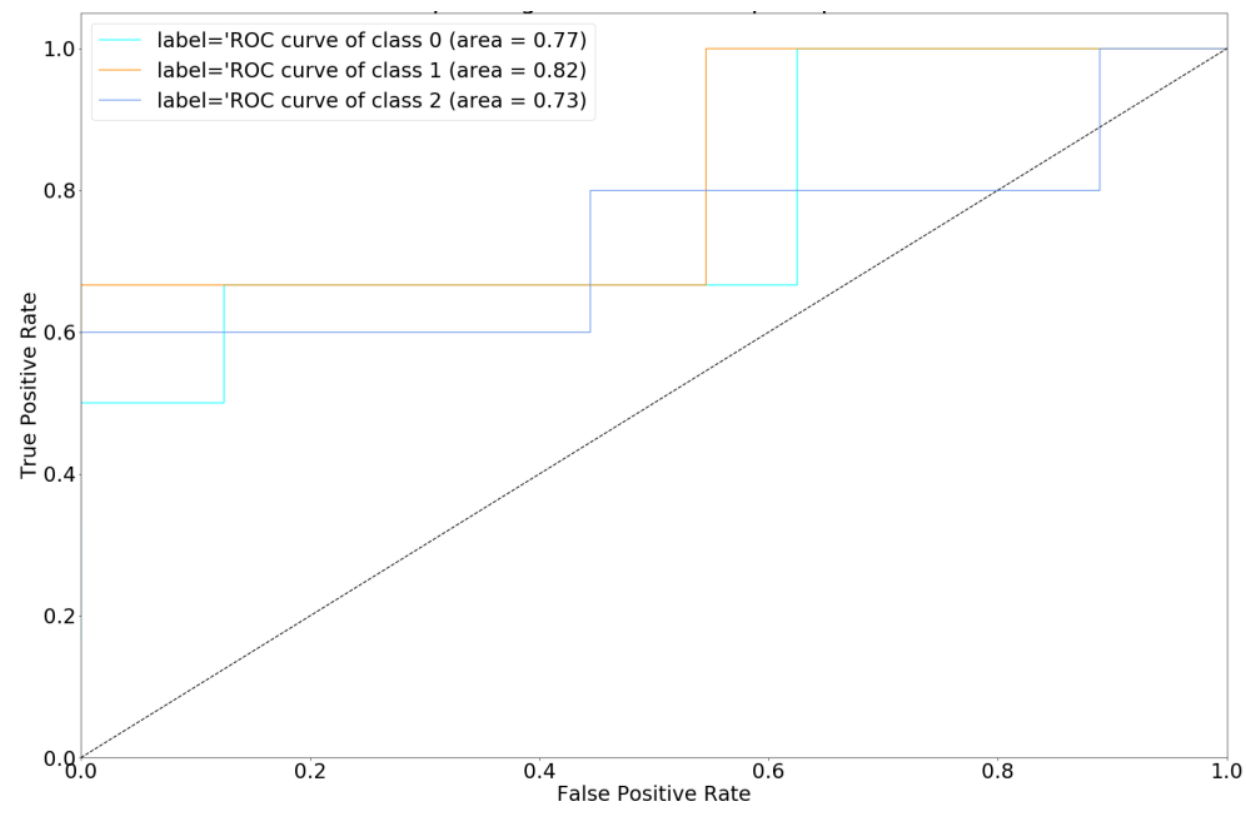

Figure 4.15 ROC of Different Classes Obtained by Perceptron Classifier

Figure 4.15 demonstrated the TP vs FP rates of all the 3 classes when using perceptron algorithm. From the graph it could be observed that class 2 had the lowest accuracy as it touches the 0.5 random guess line. Moreover, the AUC of class 2 was the lowest among all the 3 classes which could also mean that class 2 had the lowest accuracy among all the 3. Class 0 also demonstrated to be the second AUC area; however, it also went dangerously close to 0.5 which could be interpreted that, some values might have been incorrectly classified. ROC curve of class 1 was the best and also had the highest accuracy in all the three classes. 


\subsection{Medical Application}

In practice, physicians would opt for predictive model with near to $100 \%$ sensitivity at the cost of specificity and accuracy. This would be highly important to consider when conducting modeling. To integrate the model into clinical practice, the results need to be tested in a prospective study to be compared with invasive ICP monitoring. If enough clinical evidence is obtained to justify and support the usage of the predictive model in clinical practice, such algorithm can be applied to any patient with acute brain injury (e.g. trauma, stroke, hemorrhage, etc) who is not candidate for emergent surgical intervention or ventricular drain placement. Such patients would undergo monitoring with the predictive model in ICU setting and whenever the model alarms a possible rise in ICP (high sensitivity), an invasive ICP monitoring can be placed to confirm the diagnosis (high specificity) and treat the patient accordingly.

This model could prevent the universal placement of ICP monitoring in all patients suspicious for developing ICP crisis and prevent catheter complications (e.g. intracerebral hemorrhage, infection, etc.). The predictive model is not expected to function as a sole source of decision making for treatment plan and noting that the physiological signals / vital time series are routinely available and getting collected from the traumatic brain injured patients and those signals are not hazardous or complicated to get collected for the patients, modeling ICP changes from the available body signals is cost-effective and such models could be integrated into a decision support system in future after passing the requirements mentioned above.

\subsection{Discussion}

The predictive model of the present research was compared with the result of the model proposed by Guiza et al. [108] that was developed based on mean arterial pressure, cerebral perfusion pressure and mean arterial pressure time series, collected using invasive technique, to forecast future ICP signals. Despite the relatively successful outcomes, their model could not work towards non-invasive prediction due to including ICP and CPP history in feature space. Comparison of the predicted values of AUROC scores on the ICP forecasting problem between 
the present model and that of Guiza et al. demonstrated that the proposed model in this study is performing better as shown in table 4.18 .

The proposed model of this study was also compared with the model developed by Hu et al. [71] which was primarily based on the 24 MOCAIP (Morphological clustering) metrics which characterized the form of the ICP pulse. Comparison of the results between the present model, that of Guiza et al. [108] and Hu et al.[71] at $t=10$ minutes shown in table 4.18.

Comparison between the predictions of this study compared with Hüser et al.'s model [2] shown in table 4.18 illustrated that the proposed model in this study has increased AUROC metric by 5 percent. It is highly important to note that the 5 percent improvement of AUROC metric is achieved without including ICP and CPP (cerebral signal) as features in the non-invasive predictive model of this study. The proposed study has find mapping between heart channel and respiration channel to cerebral channel by two components extracted from the correlated body signals with ICP and being able to map them to normal ICP / Intracranial hypertension status using the state of the art deep learning model. However, in Hüser's predictive model the future status of ICP was predicted by its history and the methodology by that study use ensemble extremely randomized decision tree and SVM.

Hüser et al. [2], [5] also developed a non-invasive predictive ICP model with the same input space excluding ICP and CPP to predict the current exact value of intracranial hypertension mean via a supervised regressive model as explained in chapter 2 . Hüser et al. conducted 10 fold cross-validation and figured a mean absolute error of $3.84 \mathrm{mmHg}$. The author reported that the exact current mean ICP was not achieved in his experiments.

Kashif et al. [73] developed a technique to estimate the current ICP. Their model utilized ABP (in 60 beat time intervals) and Transcranial Doppler readings of cerebral blood flow velocity. Evaluation of their model using the data collected from 37 TBI patients showed the error of 1.5 $\mathrm{mmHg} \pm 5.9 \mathrm{mmHg}$ variance. However, the positive point about their model was that it did not require a calibration.

The studies by Hüser et al [2] and Kashif et.al [73] conducted analysis on mean ICP prediction including the features from ICP or CPP values in the predictive model. These models 
could serve as "automation" of the current setting and the models are dependent of ICP signal as the input.

The experiments conducted by them are only comparable to the first model proposed in this research. The second proposed model in this research have conducted an unsupervised approach to cluster correlating body signals with ICP. This would be to evaluate the possibility of such structures through body signals and whether the information regarding status of ICP could be found. So K-means clustering result based on the optimum number of K versus the sum of the squared error resulted in 3 clusters. Each cluster received a label based on the members grouped in each cluster and the comparison between changes of members in different clusters. The result was evaluated with various supervised approaches and the best learning model based on perceptron reported an overall MSE equal to 0.19. The "normal" label received an AUROC score of 0.77, the "pre-raised" label received an AUROC of 0.82 and the "Intracranial hypertension" states received an AUROC of 0.73.

Table 4.18 Comparison with the literature

\begin{tabular}{|c|c|}
\hline Model & AUROC \\
\hline Non-invasive proposed model & 0.86 \\
\hline Invasive Guiza et al. model & 0.78 \\
\hline Invasive Hüser et al. model & 0.81 \\
\hline Invasive Hu et al. model & 0.74 \\
\hline
\end{tabular}




\subsection{Summary}

Prediction of non-invasive ICP is crucially important in the management of TBI patients. The present study evaluated the efficacy of statistical and machine learning approaches (deep learning and K-means clustering) in prediction and clustering of the intracranial hypertension status in TBI patients with respect to the vital physiological signals. This study has potentially significant clinical value in making intracranial hypertension prediction, focusing on components extracted from the physiological signals as a reliable and novel biomarker of intracranial hypertension.

The present research found significant correlations between ICP and a number of the collected biomedical vital signals from TBI patients capable to predict status of ICP for the upcoming 10 minutes. The second proposed model based on an unsupervised approach recognized the status of ICP (normal / pre-raised / raised) and was evaluated by a supervised approach. 


\section{Conclusion}

\subsection{Summary of Findings}

The principle findings of the present thesis could be summarized as follows:

i. The proposed non-invasive predictive model can be used to help answering the very common question of the medical doctors which is "Whether the ICP monitoring bolt should be placed for the patient or not". The non-invasive predictive model is able to predict the status of ICP, 10 minutes in advance without including history of ICP as the input. In other words, the future status of ICP is not modelled from its previous history; instead, it is modelled from the 2 components extracted from the other body signals. The ICP monitoring bolt could be placed only after the high ICP suspicion is warned.

ii. The first proposed machine learning model for non-invasive prediction of ICP were trained and tested successfully using correlated body signals with ICP. The model was tested using 10-fold cross validation of MIMIC II waveform database as well as the BrainIT database. It demonstrated the AUROC of 0.86 . This model was trained with available ICP labels and did not include the history of ICP or any sub derived signals from ICP such as CPP in feature engineering.

iii. The second proposed modelling was conducted with the purpose of finding structure in the correlated body signals with ICP. They were clustered and labelled. The acquired labels were validated through classification algorithms. The perceptron classifier demonstrated the AUROC of 0.82 for the acquired label of "Pre-raised ICP", AUROC of 0.77 for the acquired label of "Normal ICP" and AUROC of 0.73 for the acquired label of "Intracranial Hypertension/ raised ICP".

iv. Three labels of normal, pre raised and raised ICP were defined allowing to detect status of ICP from physiological channels.

v. The present study utilized statistical, principal component analysis and machine learning methodology to conduct deep investigation through body signals such as 
different leads of heart and reparation system and to find correlation with ICP and anticipate ICP via predictive modeling.

vi. The predictive modelling of the present study is able to anticipate the forthcoming status of ICP up to 10 minutes in advance (without including the history of ICP in the feature space). This is a critical finding which can help accelerate the medical decision making and prevent from the fatal gap between diagnosis and decision making stages.

vii. Modelling ICP from routinely collected physiological channels is feasible and cost effective and more importantly a lifesaving technique. The proposed forecasting model provides time for clinicians to make appropriate decision and avoid secondary brain damage observed in traumatic brain injured patients

viii. It was found that while the heart and respiration systems have remarkable relation with ICP, interpreted as "associations"; other parameters such as tumors, stroke, seizures, infections etc. may have a "causality" relationship with ICP. This fact, makes any clinical study very hard to analyze.

\subsection{Contributions}

The main contributions are:

- Finding correlations between body signals through different leads of heart ( such as ECG segment III and IV) and respiration system with ICP and extracting two components from them for modelling ICP.

- Building a non-invasive forecasting model for prediction of intracranial hypertension status with a horizon of $t=10$ minutes, with the sensitivity of 94.4 through a two-component solution from the correlated body signals with ICP.

- A non-invasive ICP status detection model based on clustering, labelling and evaluating via classification algorithms. The best accuracy of labelling result was demonstrated by the perceptron algorithm as discussed in summary of findings.

- A detailed evaluation of the importance of different physiological and clinical features with respect to ICP. 
- Parallel visualization of body signals corresponding to changes of ICP.

\subsection{Publications}

- P. Naraei, A. Abhari, and A. Sadeghian, "Application of Multilayer Perceptron Neural Networks and Support Vector Machines in Classification of Healthcare Data," in Proc. of the IEEE Future Technologies conference, pp. 848 - 852, Dec. 2016.

- P. Naraei, M. Nouri, A. Sadeghian "Toward Learning Intracranial Hypertension through Physiological Features: A Statistical and Machine Learning Approach, "in Proc. Of IEEE Intelligent System Conference, pp.395 - 399, Oct 2017.

- P. Naraei, A. Sadeghian, "A PCA Based Feature Reduction in Intracranial Hypertension Analysis," in Proc. Of the IEEE Canadian Conference on Electrical and Computer Engineering (CCECE), pp.1-6, Sep 2017.

- P. Naraei, M. Kenez, A. Sadeghian, "A Hybrid Wavelet-Based K-Means Clustering Approach to Detect Intracranial Hypertension ", in Proc. Of the IEEE International Humanitarian Technology Conference (IHTC), pp.21-25, Oct 2017.

\subsection{Future Work}

The primary results obtained in this research is very promising. In future the predictive model should be tested in multiple large-scale studies to confirm their sensitivity and reliability to be incorporated into the clinical settings. This would be to achieve the top most future goal and to build a decision support tool that could be incorporated to the current clinical settings to help neurosurgeons in the surgery decision making.

It should be noted that when conducting forecasting modeling in clinical setup, the emphasis on "Sensitivity" of the model is higher than the accuracy. Since gathering the data from patients with specific characteristic under the same condition is a time consuming task, reliable biomedical data sets are generally very limited and this presents a common challenge in analysis of 
physiological data sets. As ICP is very predictive of the patient outcome, a good future direction would be to associate the findings of this thesis with patient outcome and predicting Glasgow Coma Scale of patients. In addition, a recurrent neural network could be a good solution to infer insights into the wavelet images. 


\section{Appendix}

\section{Appendix A. Wavelet Plots: samples of pre-raised ICH}

\#Description: Appendix A, demonstrates samples of pre-raised ICP in body signals. Together with "normal" samples and "ICH" samples, they are provided as the input to K-means clustering algorithm.

\section{ICP on individual 93}

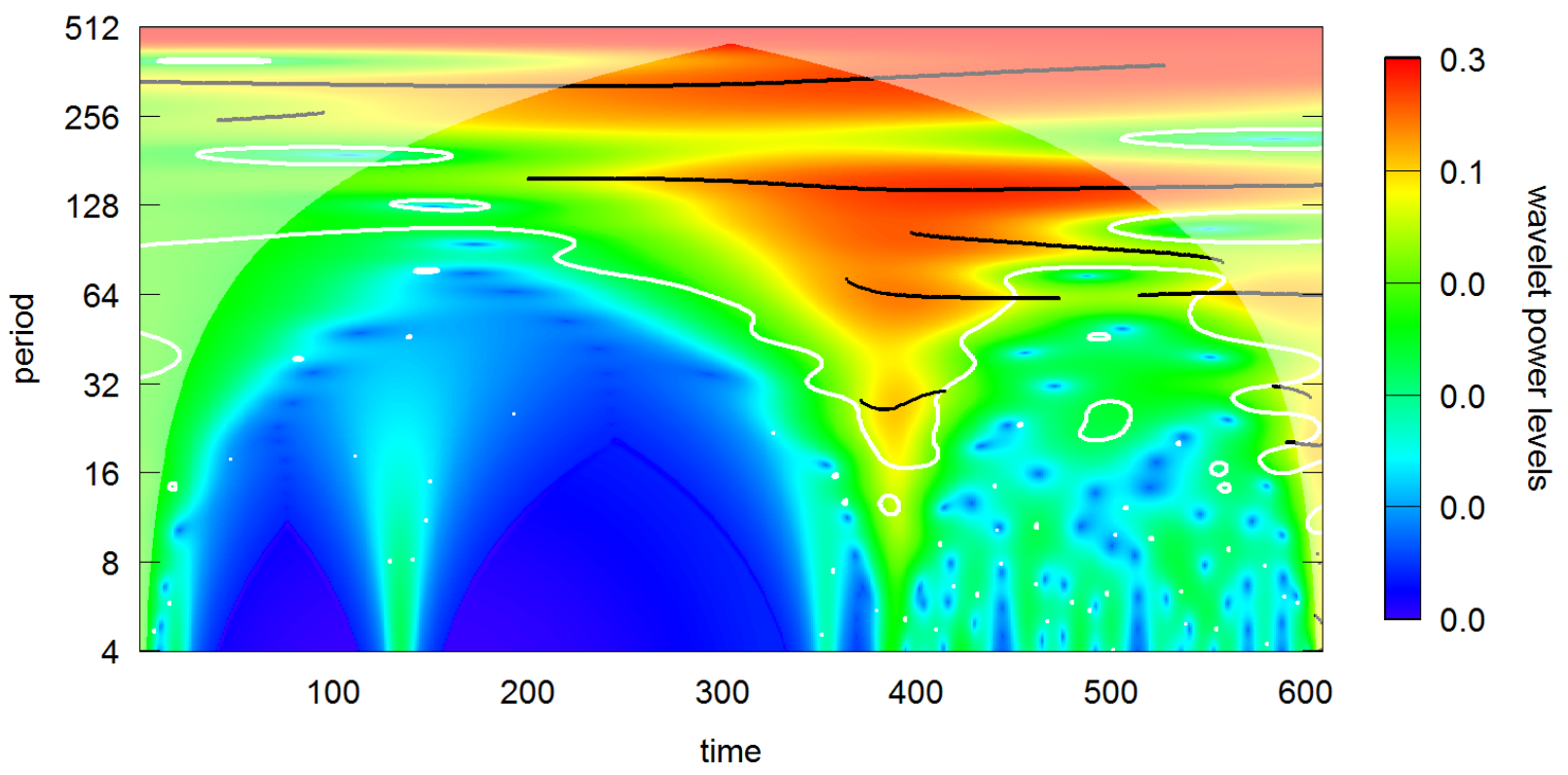




\section{Heart Rate on individual 93}

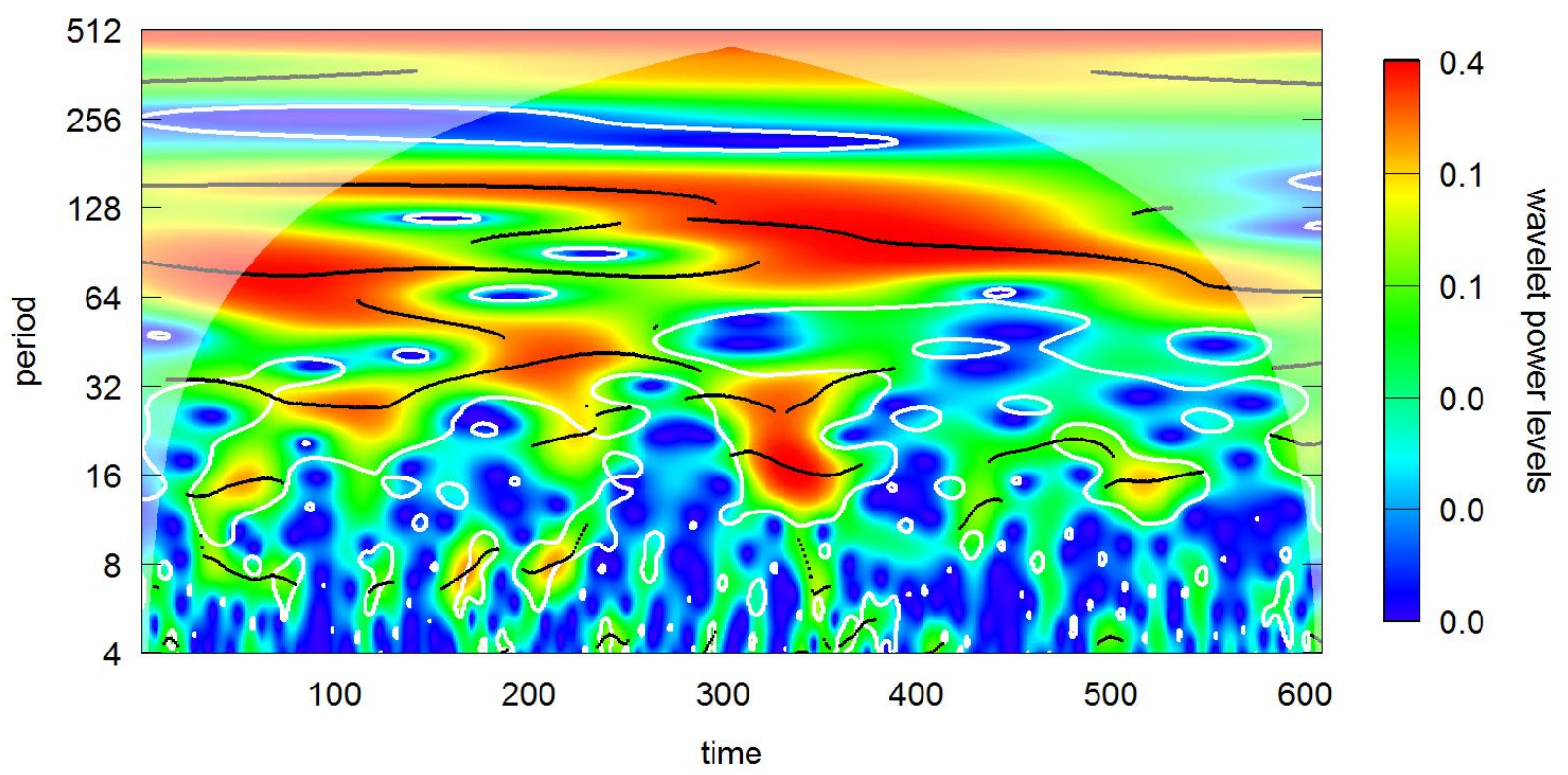

ABP dias on individual 93

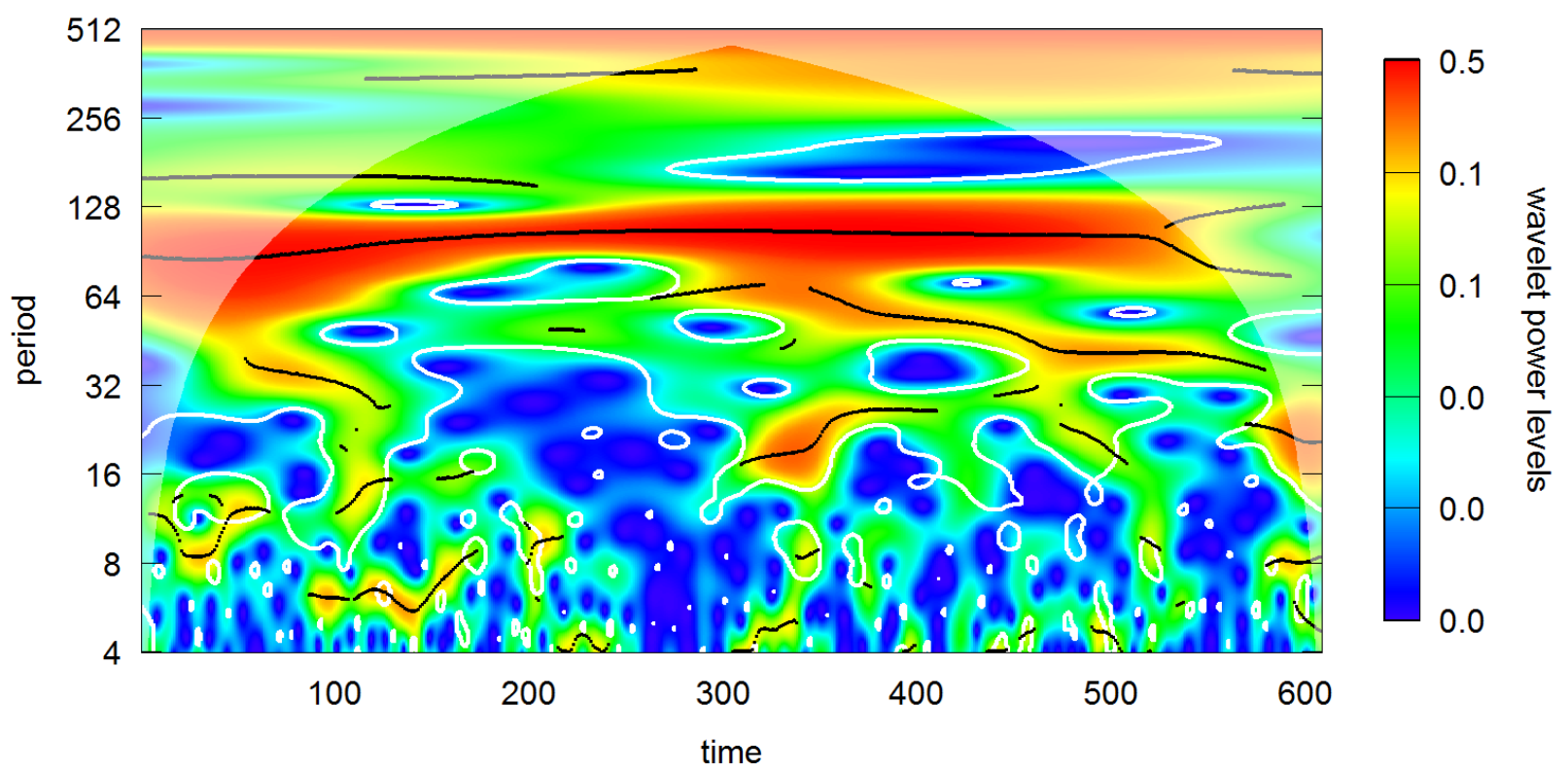




\section{ABP mean on individual 93}

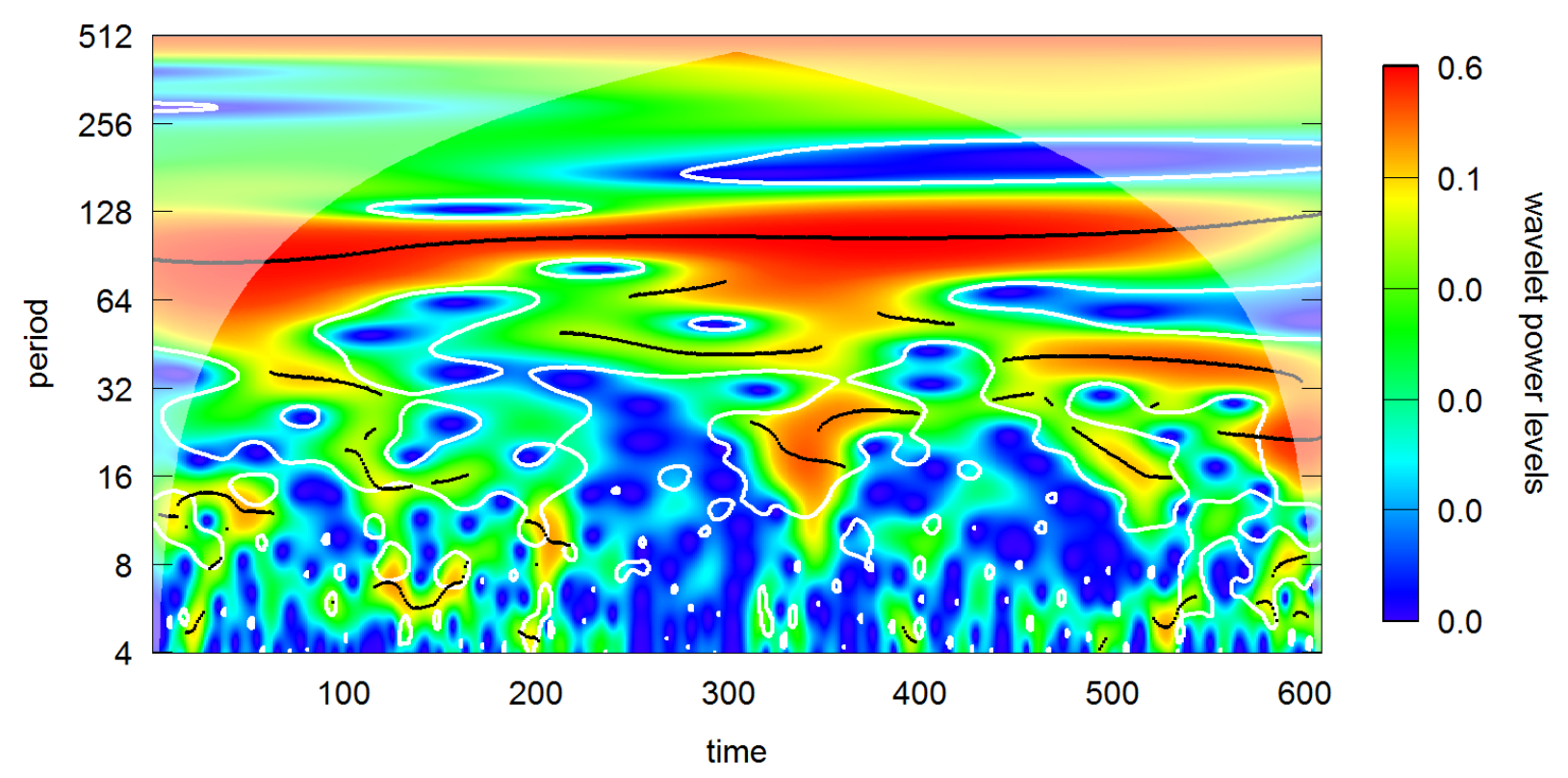

\section{Appendix B. Extraction of the raised episodes of ICP}

\#Description: Appendix B, contains the script used to generate parsed files and extract ICH episodes in the correlated signals with ICP.

from datetime import datetime

file_name $=$ 's7.csv'

\# open the csv file

file $=$ open(file_name, 'r')

\# create a parsed file

parsed_file $=$ open $($ parsed ' + file_name, 'w')

\# extract the header and the units

header $=$ file.readline () 


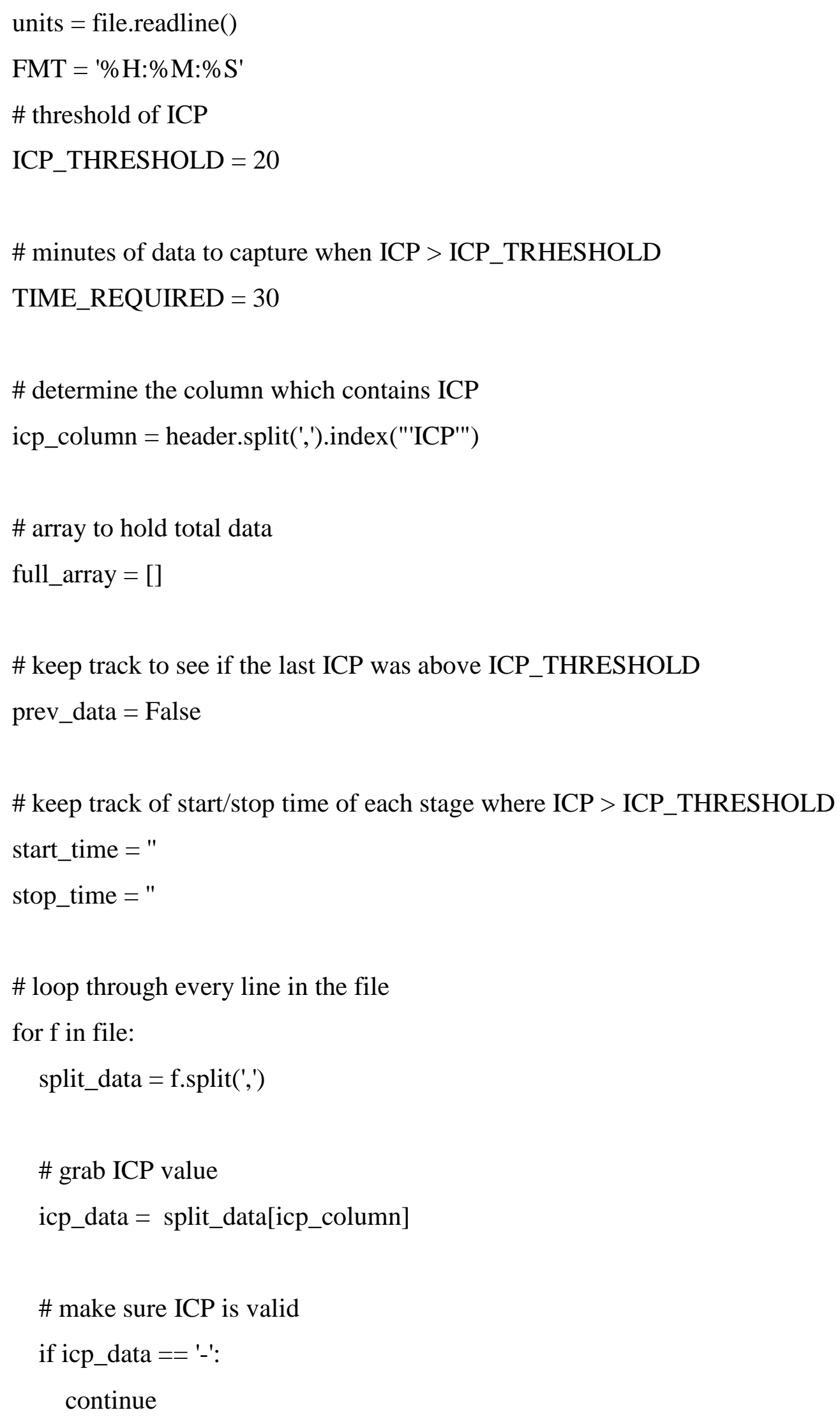




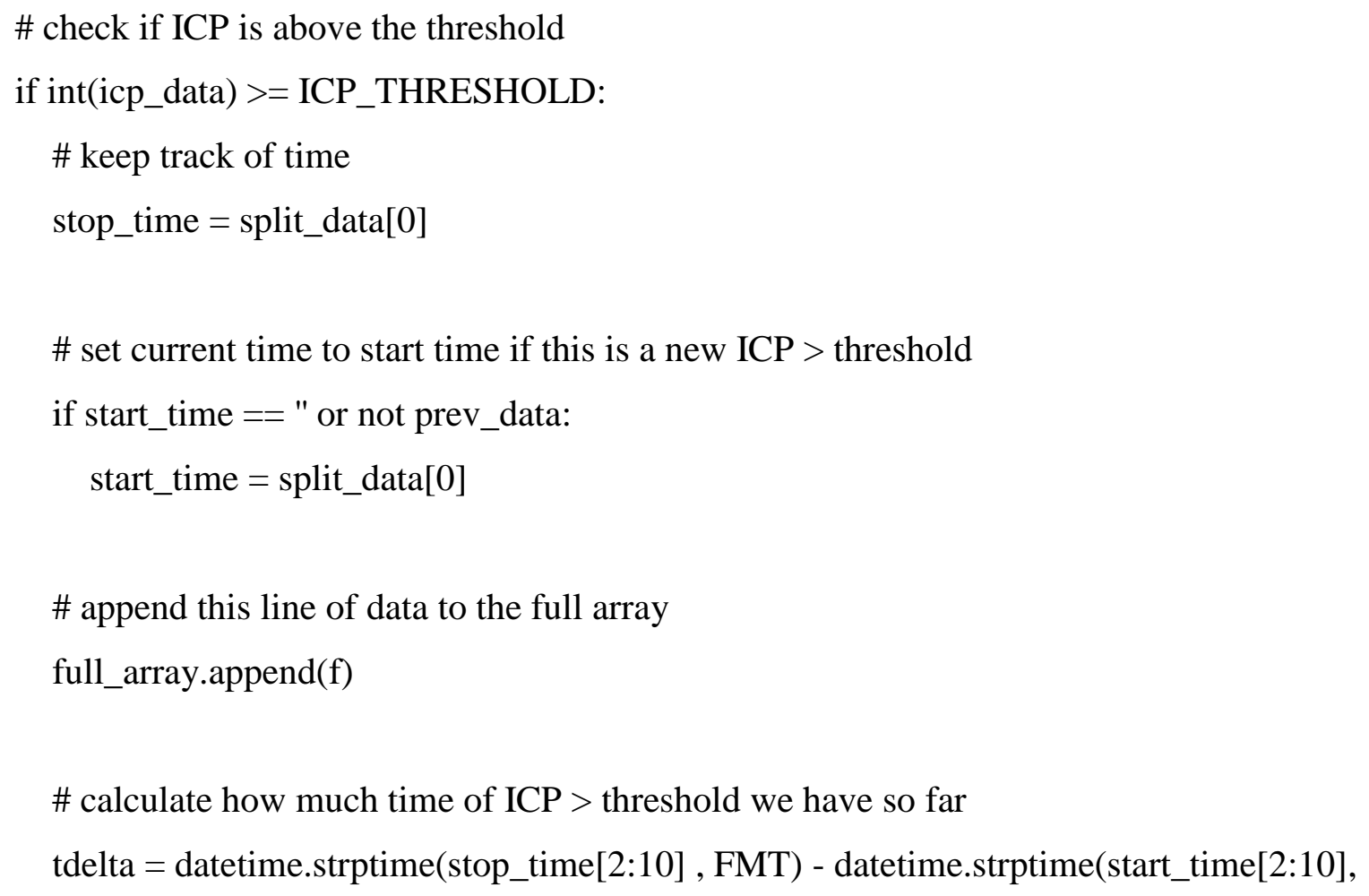

\# if ICP does not meet threshold, reset data array, and set previous ICP as false else:

prev_data $=$ False 
full_array $=[]$

\section{Appendix C. Implementation of the predictive model}

\#Description: Appendix C, contains the script used to implement the neural network model using h2o library in R.

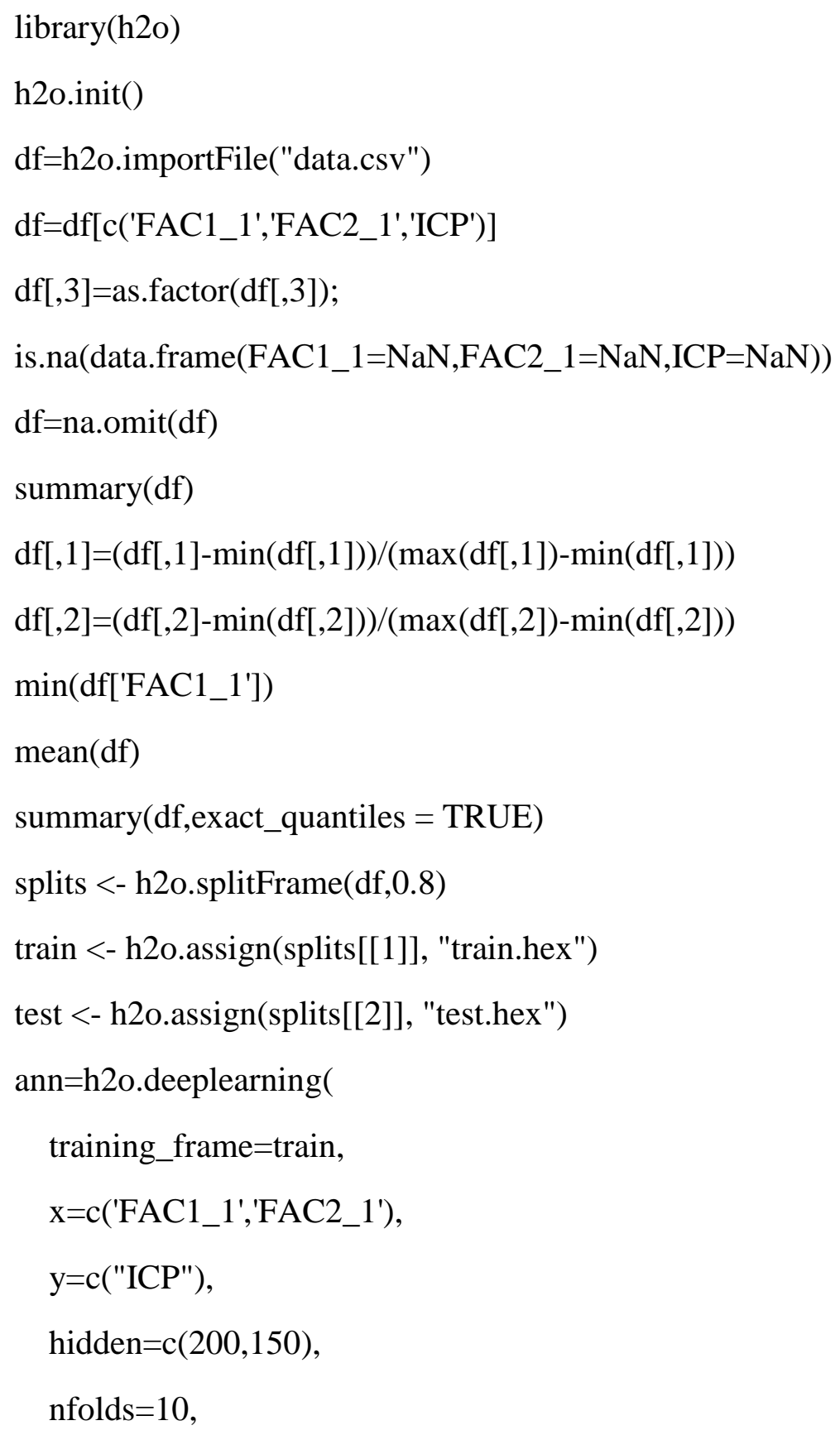




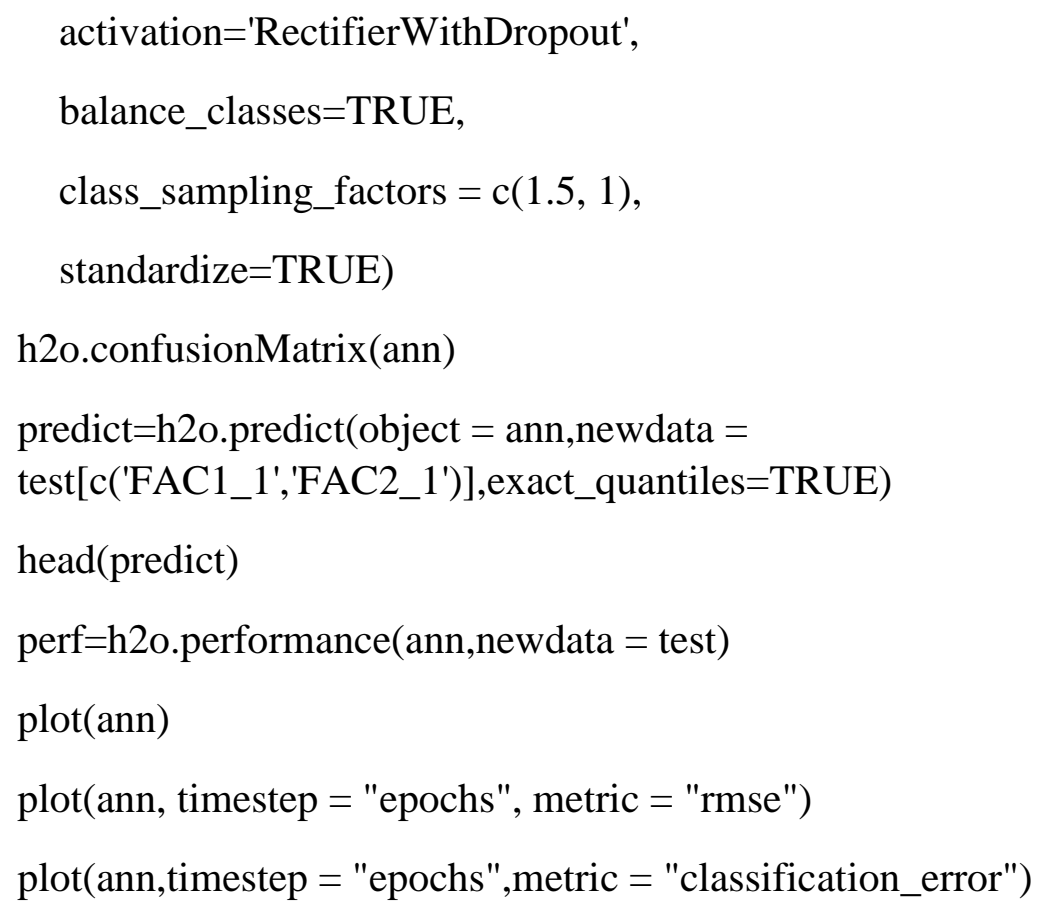

\section{Appendix D. Sample result of PCA (Fact 1 and Fact 2 as Inputs to Neural Network)}

\#Description: Appendix D, demonstrates a sample data file in which Fact 1 and Fact 2 are the two components extracted from HR, Pulse, ST V, ST III, Abp dias and Abp mean and Resp. Moreover, Fact 1 and Fact 2 are the inputs to Neural network to predict ICH.

\begin{tabular}{rrrrrrrrrr} 
HR & X.PULSE & ST.V & X.ABP.Dias & X.ABP.Mean & X.RESP & STIII & \multicolumn{2}{l}{ FAC1_1 } & FAC2_1 \\
ICH & True \\
51 & 51 & 154 & 66 & 94 & 12 & 100 & -1.41896 & 0.71344 & True \\
51 & 51 & 154 & 66 & 94 & 12 & 100 & -1.41896 & 0.71344 & True \\
51 & 51 & 154 & 66 & 94 & 12 & 100 & -1.41896 & 0.71344 & True \\
51 & 51 & 154 & 66 & 94 & 12 & 100 & -1.41896 & 0.71344 & True \\
50 & 51 & 154 & 66 & 94 & 12 & 100 & -1.44126 & 0.72368 & True \\
50 & 51 & 154 & 66 & 94 & 12 & 100 & -1.44126 & 0.72368 & 0.72368 \\
50 & 51 & 154 & 66 & 94 & 12 & 100 & -1.44126 & True \\
51 & 51 & 154 & 66 & 94 & 12 & 100 & -1.41896 & 0.71344 & True \\
51 & 51 & 154 & 66 & 94 & 12 & 100 & -1.41896 & 0.71344 & True \\
51 & 51 & 154 & 66 & 94 & 12 & 100 & -1.41896 & 0.71344 & True \\
51 & 51 & 154 & 66 & 94 & 12 & 100 & -1.41896 & 0.71344 & True \\
51 & 51 & 154 & 66 & 94 & 12 & 100 & -1.41896 & 0.71344 & False
\end{tabular}


Result:

\begin{tabular}{|r|r|}
\hline Q Code.R & totall $\times$ \\
\hline & Physiol \\
\hline Patient_Id & attr_importance \\
\hline Daily_Fluid_Input & 0.00000000 \\
\hline Daily_Fluid_Output & 0.04815306 \\
\hline BPm & 0.04815306 \\
\hline TC & 0.12821082 \\
\hline SaO2 & 0.36967054 \\
\hline GCS_Eye & 0.03107773 \\
\hline GCS_Motor & 0.00000000 \\
\hline GCS_Verbal & 0.00000000 \\
\hline FiO2 & 0.00000000 \\
\hline
\end{tabular}

Appendix F. Selecting patients with normal lead before episodes of highICP (Checking for 5, 10, 15 and 20 minutes' lead samples)

\#Description: Appendix F, demonstrates the script written in $\mathrm{R}$ to find the maximum available lead time detectable in normal samples that are followed by episodes of highICP right after.

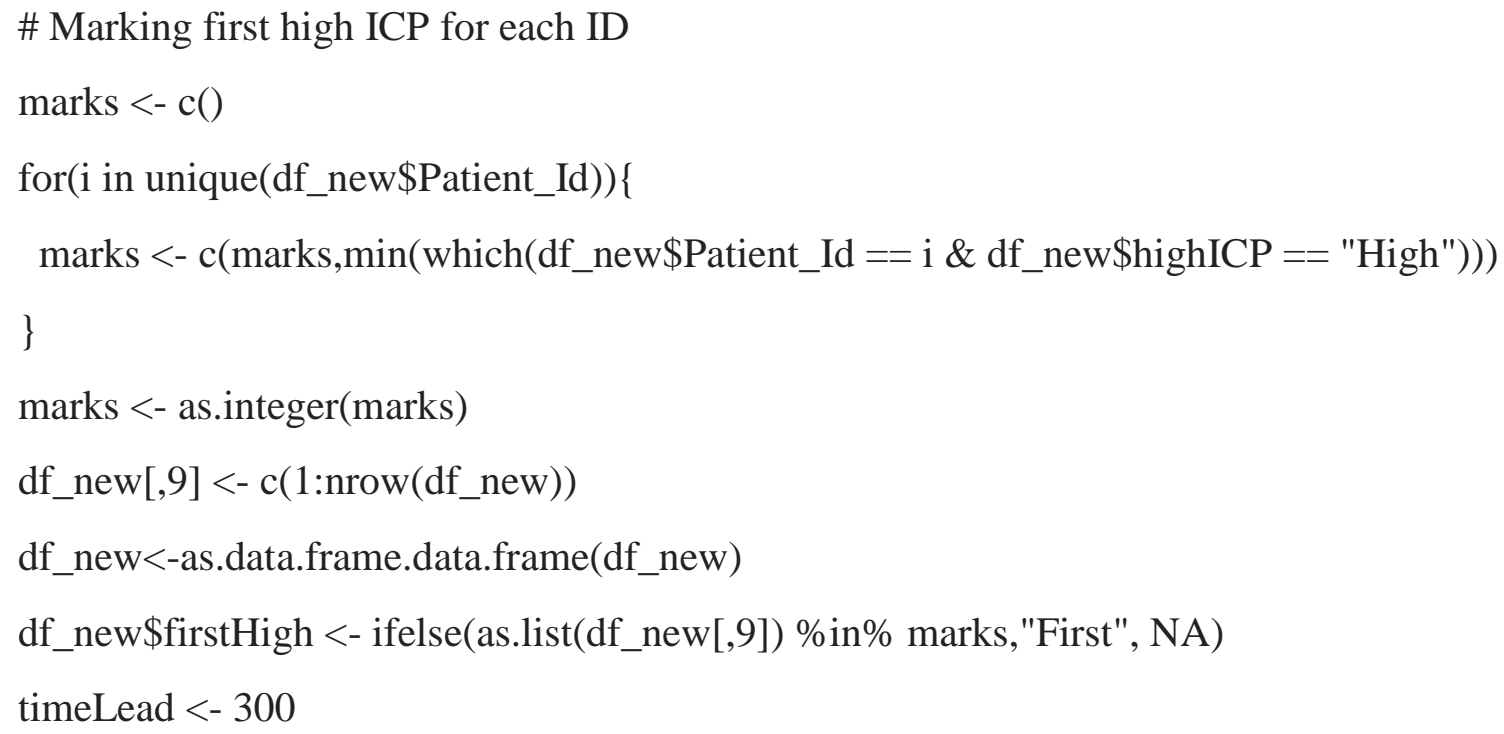




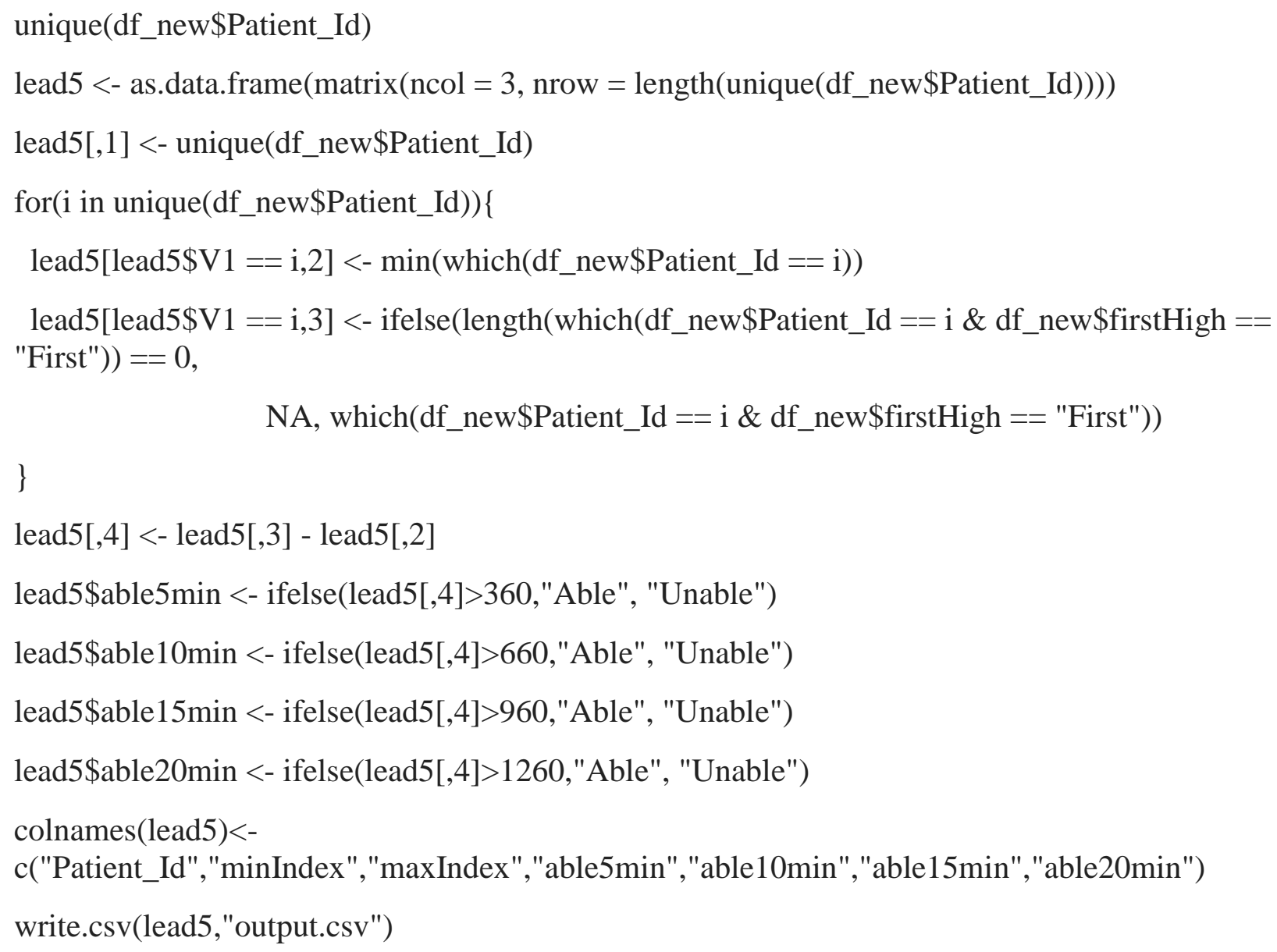

\section{Appendix G. K-means Clustering on Wavelet plots}

Description: Appendix G, demonstrates the script written in Python to conduct K-means clustering on the wavelet plots.

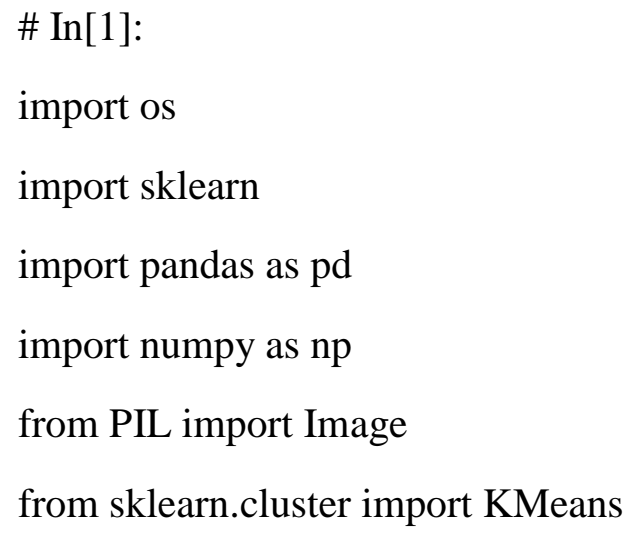


import cv2

from sklearn.metrics import silhouette_samples, silhouette_score

import matplotlib.pyplot as plt

import pyximport; pyximport.install()

from matplotlib.pylab import rcParams

rcParams['figure.figsize'] $=15,6$

import matplotlib.cm as $\mathrm{cm}$

\# In[141]:

DATA_URL=os.path.join("Dataset")

SORTED_DATA_URL=os.path.join("SortedProjectImages");

\# In[120]:

RESULT_URL=os.path.join("kmeansV2Result")

\# In[3]:

signals=["pulse","heartRate","resp","pvc","spo2","abp","abpDias","abpSys","st3",,"st5"]

\# In[4]:

patientNumbers=os.listdir(DATA_URL)

\# In[5]:

n_cluster $=3$

NUM_SIGNAL=10;

IMAGE_SIZE=224;

IMAGE_DIM=3;

len(patientNumbers)

\# \# Gathering the data

\# In[104]:

data $=[]$

label=[]

fileNames $=[]$; 
for num in patientNumbers:

baseUrl=os.path.join(DATA_URL,num);

temp $=[]$

for signal in signals:

tempFileName=signal+"_"+num+".png";

if(os.path.exists(os.path.join(baseUrl,tempFileName))==True):

image $=c v 2$. imread (os.path.join(os.path.join(baseUrl,tempFileName) $)$ )

fileNames.append(tempFileName)

data.append(image.flatten())

label.append(num)

\# In[105]:

len(data)

\# In[106]:

data $=$ np.array $($ data $)$

\# In[107]:

data.shape

\# \# doing k-means

\# In[112]:

KmeansClustering=KMeans(n_clusters=3, init='k-means++', n_init=100, max_iter=3000) \# In[113]:

KmeansClustering.fit(data)

\# In[114]:

output_labels=KmeansClustering.labels_

\# \# Creating Cluster Result Folder

\# In[135]:

os.mkdir(os.path.join(RESULT_URL,"cluster_0"))

os.mkdir(os.path.join(RESULT_URL,"cluster_1")) 


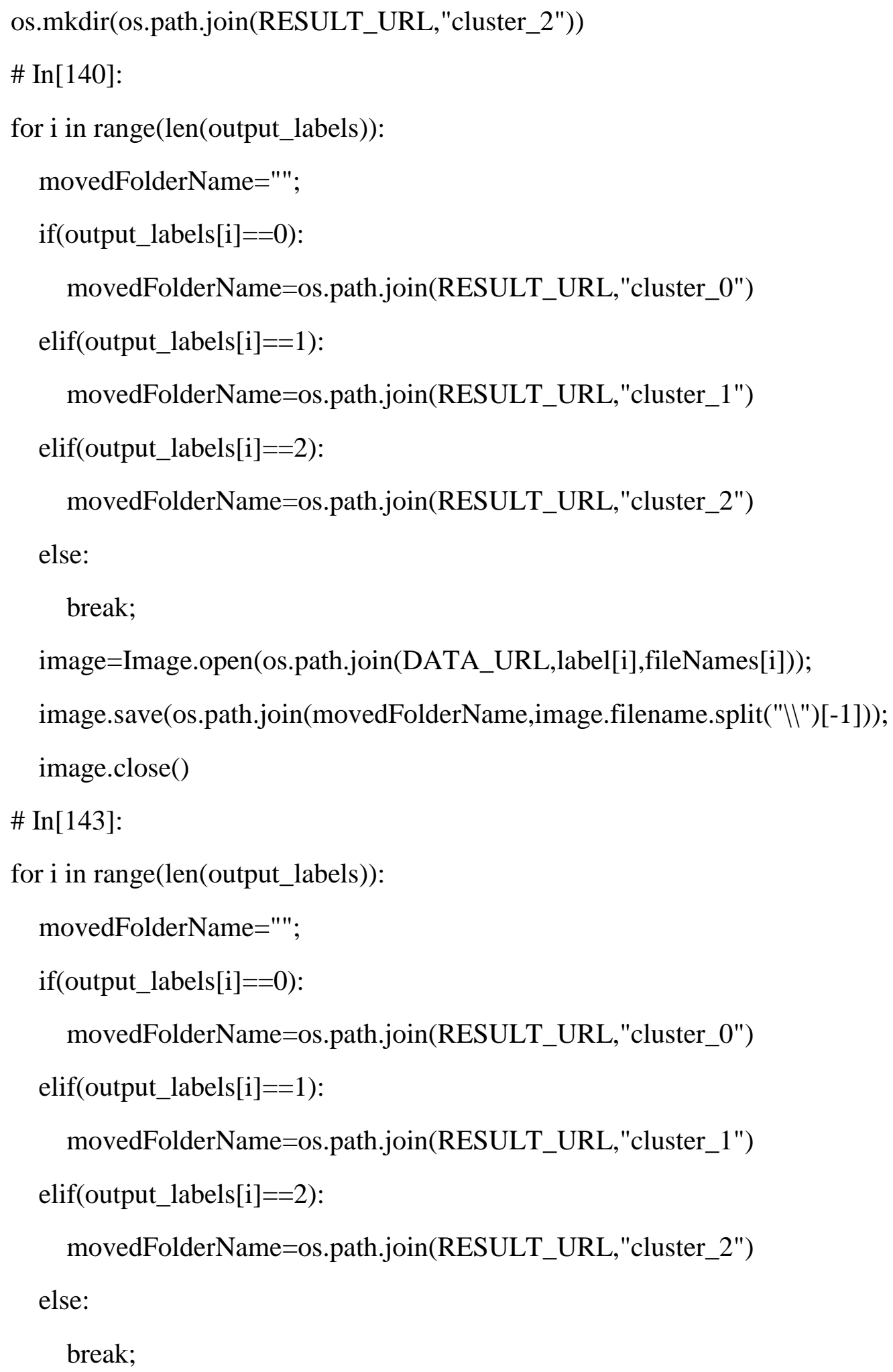


image.save(os.path.join(movedFolderName,fileNames[i]))

image.close ();

\section{Appendix H. Classification of K-means Result}

\#Description: Appendix H, demonstrates the script written in Python to conduct classification on the K-means result.

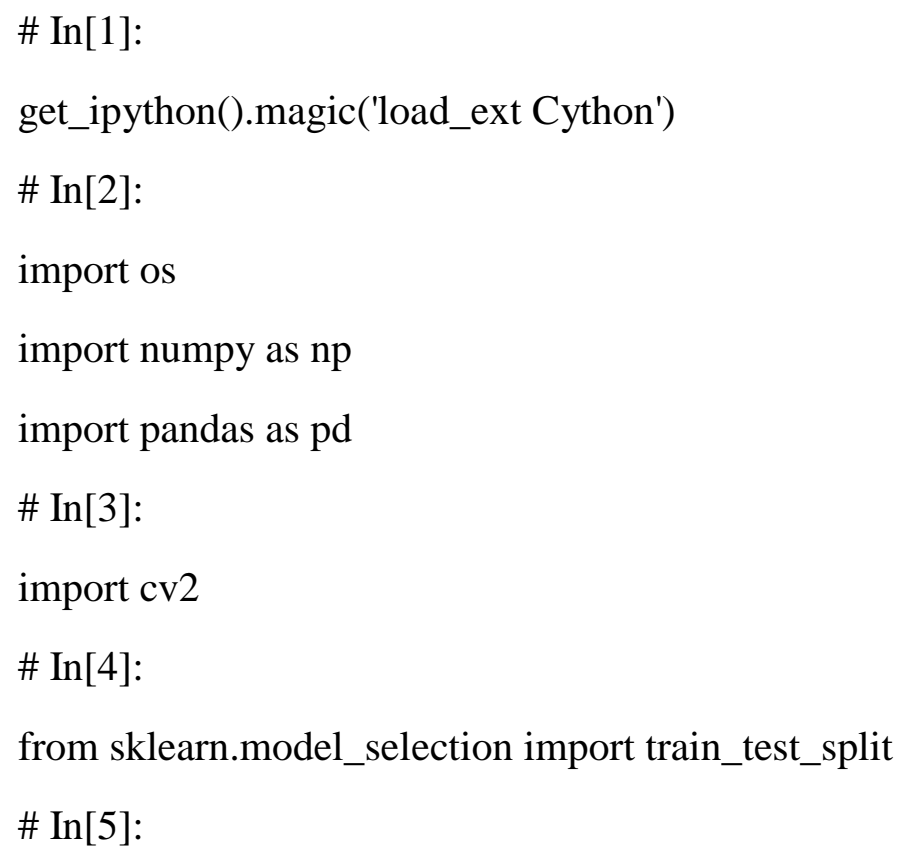


\# In[9]:

from sklearn.naive_bayes import BernoulliNB

from sklearn.naive_bayes import GaussianNB

from sklearn.naive_bayes import MultinomialNB

\# In[10]:

from sklearn.feature_selection import RFE

from sklearn.feature_selection import SelectKBest

from sklearn.feature_selection import chi2

from sklearn.decomposition import PCA

\# In[296]:

from sklearn.metrics import confusion_matrix

from sklearn.metrics import classification_report

from sklearn.metrics import roc_auc_score

from sklearn.metrics import recall_score

from sklearn.metrics import precision_score

from sklearn.metrics import f1_score

from sklearn.metrics import roc_curve

\# $\operatorname{In}[322]:$

from itertools import cycle

\# In[151]:

from sklearn.preprocessing import label_binarize

\# In[50]:

import matplotlib.pyplot as plt

\# $\operatorname{In}[501]:$

from pylab import rcParams

rcParams['figure.figsize'] $=30,20$

rcParams.update(\{'font.size': 30$\})$

\# In[244]: 


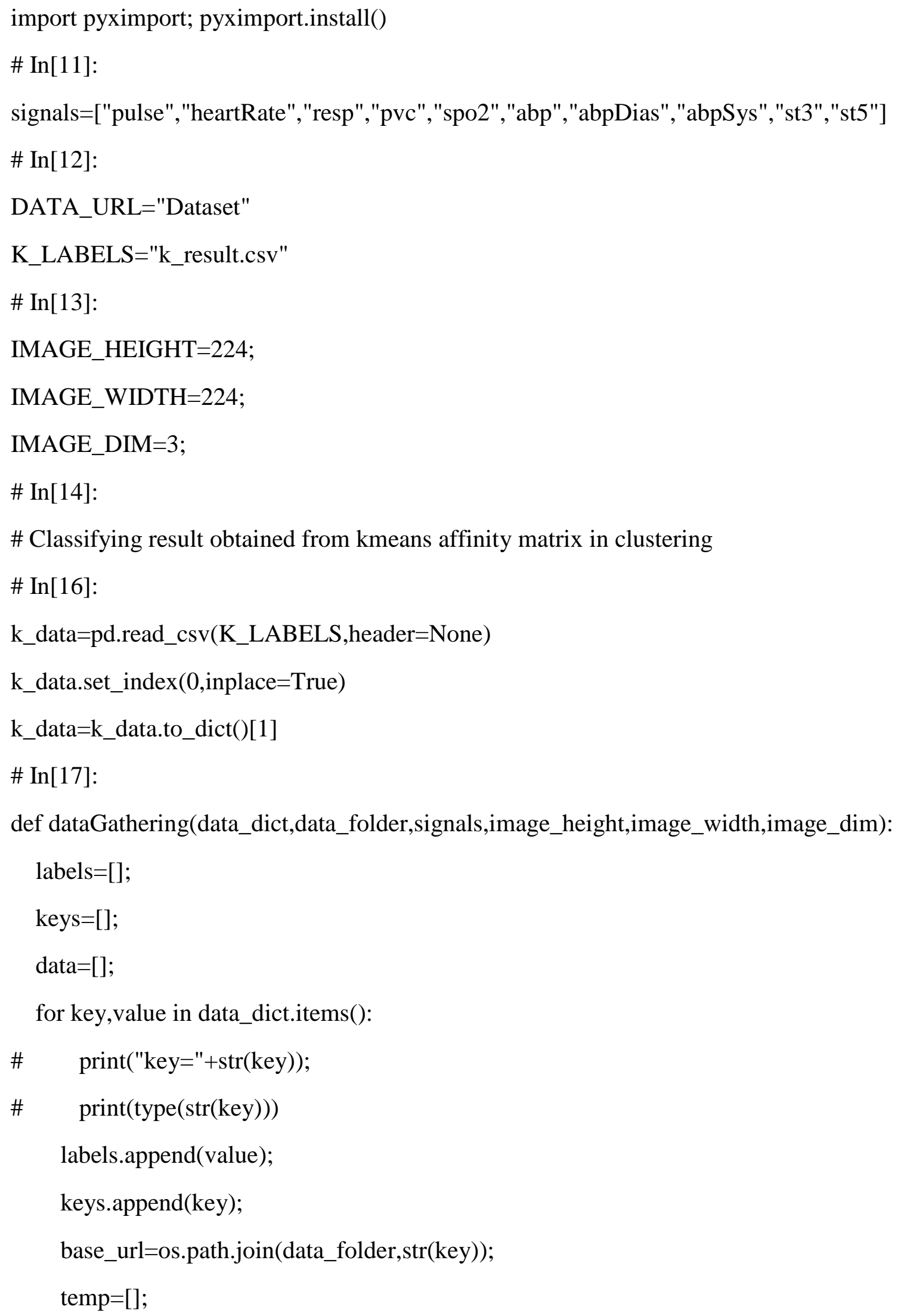


for signal in signals:

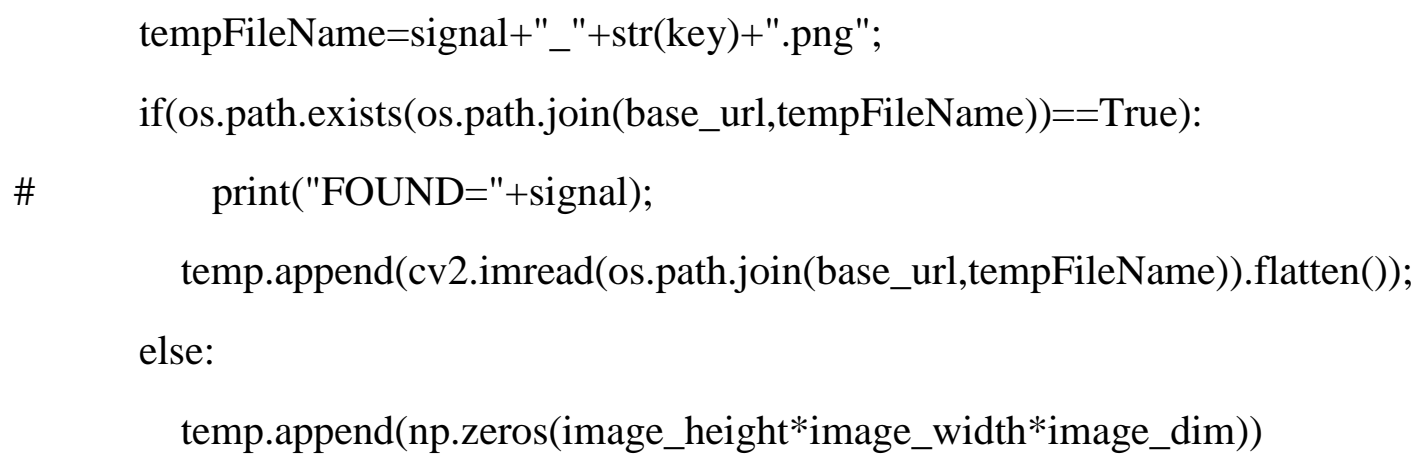


return np.mean(y_pred==ytest),y_pred,y_decision

\# In[277]:

def performPerceptron(Xtrain,ytrain,Xtest,ytest):

percep $=$ Perceptron () ;

percep.fit(Xtrain,ytrain)

y_pred=percep.predict(Xtest)

y_decision=percep.decision_function(Xtest)

return np.mean(y_pred==ytest),y_pred,y_decision

\# $\operatorname{In}[461]:$

def performRandomForestClassifier(Xtrain,ytrain,Xtest,ytest):

randomForest=RandomForestClassifier $\left(\mathrm{n} \_\right.$jobs $\left.=-1\right)$

randomForest.fit(Xtrain,ytrain)

y_pred=randomForest.predict(Xtest)

y_decision=randomForest.predict_proba(Xtest)

return np.mean(y_pred==ytest),y_pred,label_binarize(y_pred,classes=[0,1,2])

def performExtraTreeClassifier(Xtrain,ytrain,Xtest,ytest):

extraTree $=$ ExtraTreesClassifier $\left(n_{-}\right.$jobs $\left.=-1\right)$

extraTree.fit(Xtrain,ytrain)

y_pred=extraTree.predict(Xtest)

y_decision=extraTree.predict_proba(Xtest)

return np.mean(y_pred==ytest),y_pred,label_binarize(y_pred,classes=[0,1,2])

\# In[462]:

def performGaussianNB(Xtrain,ytrain,Xtest,ytest):

gaussianNB=GaussianNB () ;

gaussianNB.fit(Xtrain,ytrain);

y_pred=gaussianNB.predict(Xtest)

y_decision=gaussianNB.predict_proba(Xtest) 
return np.mean(y_pred==ytest),y_pred,y_decision

def performMultinomailNB(Xtrain,ytrain,Xtest,ytest):

$\mathrm{pNb}=$ MultinomialNB ()$;$

pNb.fit(Xtrain,ytrain);

y_pred=pNb.predict(Xtest)

y_decision $=$ pNb.predict_proba(Xtest)

return np.mean(y_pred==ytest),y_pred,y_decision

def performBernouliNB(Xtrain,ytrain,Xtest,ytest):

bernouliNB=BernoulliNB ()

bernouliNB.fit(Xtrain,ytrain)

y_pred=bernouliNB.predict(Xtest)

y_decision=bernouliNB.predict_proba(Xtest)

return np.mean(y_pred==ytest),y_pred,y_decision

\# In[463]:

def performMLPClassifier(Xtrain,ytrain,Xtest,ytest):

mlpClassifier=nn=MLPClassifier(activation="logistic", solver="sgd")

mlpClassifier.fit(Xtrain,ytrain)

y_pred=mlpClassifier.predict(Xtest)

y_decision $=$ mlpClassifier.predict_proba $($ Xtest $)$

return np.mean(y_pred==ytest),y_pred,y_decision

\# In[464]:

def startClassification(data,labels):

result_dict $=\{\}$;

result_pred_dict $=\{\}$

result_decision_dict $=\{\}$

X_train, X_test, y_train, y_test = train_test_split(data, labels, test_size $=0.25$,random_state $=42$ );

_,X_test_temp,_y_test_temp=train_test_split(X_train,y_train,test_size=0.25,random_state=42)

X_test=np.concatenate([X_test,X_test_temp]); 
y_test=y_test+y_test_temp;

result_dict['Logistic'],result_pred_dict['Logistic'],result_decision_dict['Logistic']=performLogisti cRegression(X_train,y_train,X_test,y_test)

result_dict['stochastic'],result_pred_dict['stochastic'],result_decision_dict['stochastic']=performS GDClassifier(X_train,y_train,X_test,y_test)

result_dict['perceptron'],result_pred_dict['perceptron'],result_decision_dict['perceptron']=perform Perceptron(X_train,y_train,X_test,y_test)

result_dict['randomForest'],result_pred_dict['randomForest'],result_decision_dict['randomForest' ]=performRandomForestClassifier(X_train,y_train,X_test,y_test)

result_dict['extraTree'],result_pred_dict['extraTree'],result_decision_dict['extraTree']=performEx traTreeClassifier(X_train,y_train,X_test,y_test)

result_dict['GaussianNB'],result_pred_dict['GaussianNB'],result_decision_dict['GaussianNB']=p erformGaussianNB(X_train,y_train,X_test,y_test)

result_dict['MultinomialNB'],result_pred_dict['MultinomialNB'],result_decision_dict['Multinomi alNB']=performMultinomailNB(X_train,y_train,X_test,y_test)

result_dict['BernouliNB'],result_pred_dict['BernouliNB'],result_decision_dict['BernouliNB']=per formBernouliNB(X_train,y_train,X_test,y_test)

result_dict['MLP'],result_pred_dict['MLP'],result_decision_dict['MLP']=performMLPClassifier( X_train,y_train,X_test,y_test)

return result_dict,result_pred_dict,result_decision_dict,y_test;

\# In[466]:

k_result=startClassification(k_data,k_labels)

\# In[469]:

$\mathrm{kConfusionMatrix}=\{\}$

kFScore $=\{\}$ 


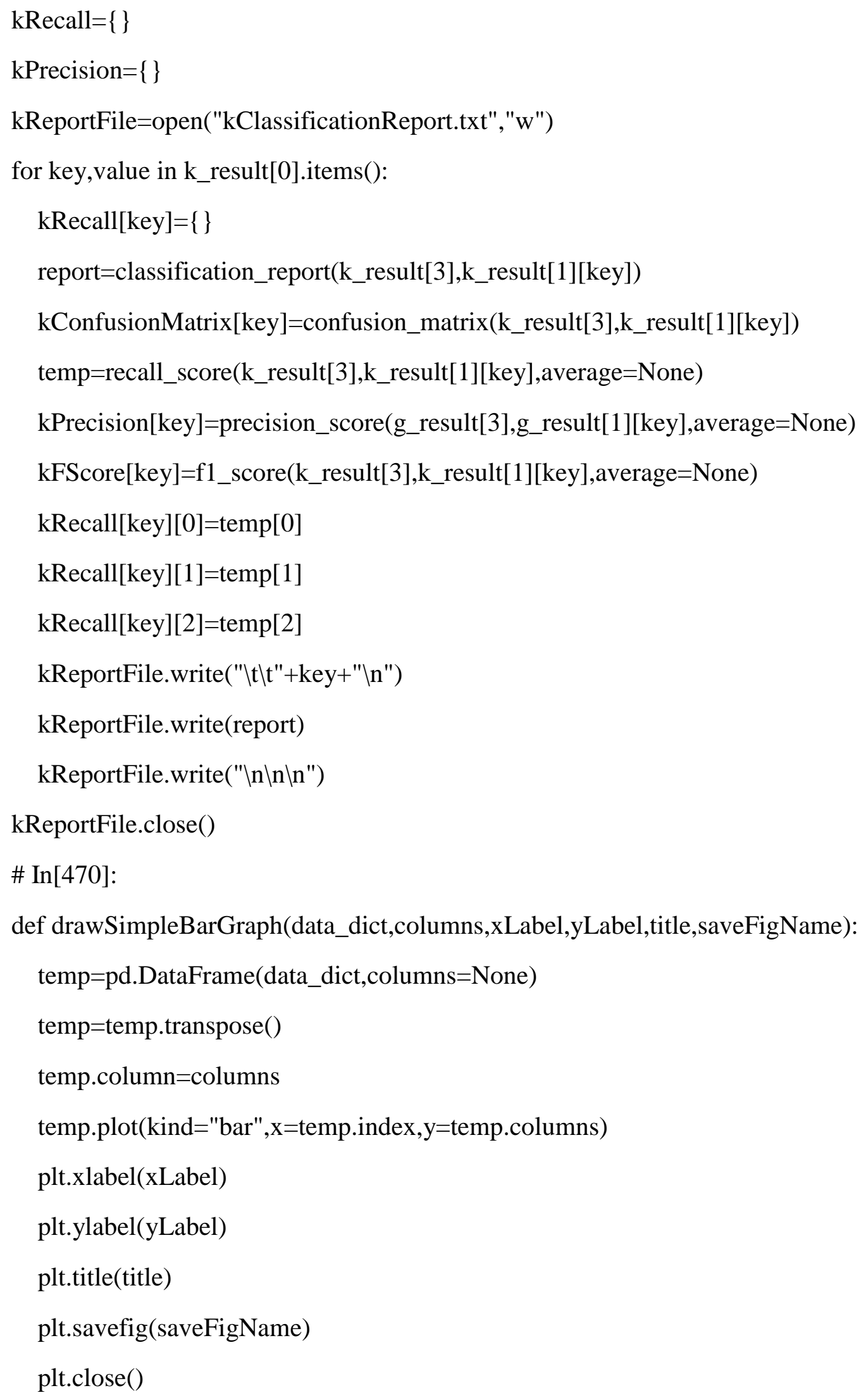




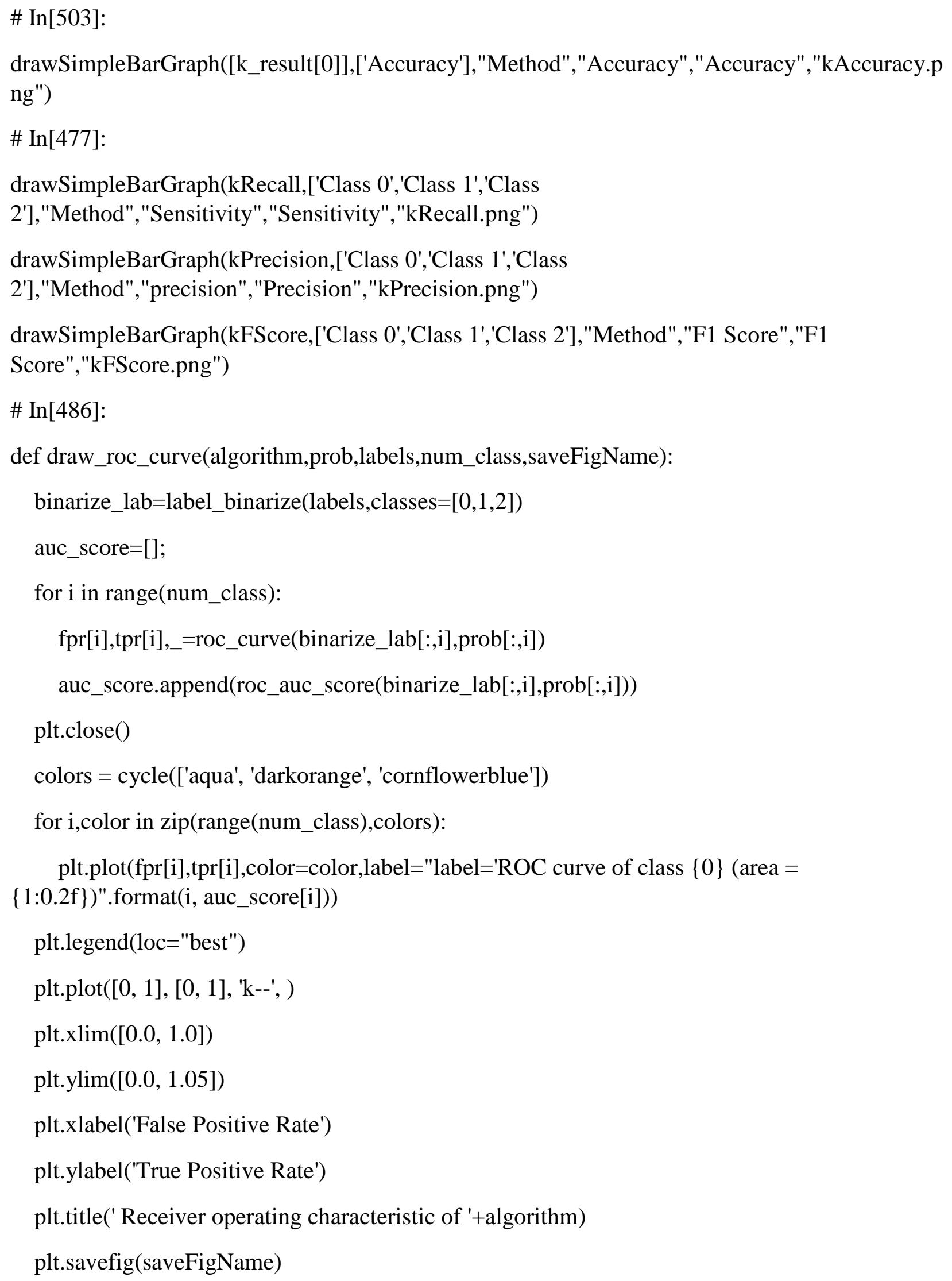


\# In[489]:

draw_roc_curve("Logistic Regression",k_result[2]['Logistic'],k_result[3],3,"k_LogisticROC") draw_roc_curve("SGD CLassifier",k_result[2]['stochastic'],k_result[3],3,"k_Stochatic_ROC") draw_roc_curve("perceptron CLassifier",k_result[2]['perceptron'],k_result[3],3,"k_Perceptron_ROC")

draw_roc_curve("MultiLayerPerceptron",k_result[2]['MLP'],k_result[3],3,"k_MLP_ROC") draw_roc_curve("BernouliNB CLassifier",k_result[2]['BernouliNB'],k_result[3],3,"k_BernouliNB_ROC") draw_roc_curve("GaussianNB CLassifier",k_result[2]['GaussianNB'],k_result[3],3,"k_GaussianNB_ROC") draw_roc_curve("MultinomialNB CLassifier",k_result[2]['MultinomialNB'],k_result[3],3,"k_MultinomialNB_ROC")

\section{Appendix I. Creating Wavelet-plots}

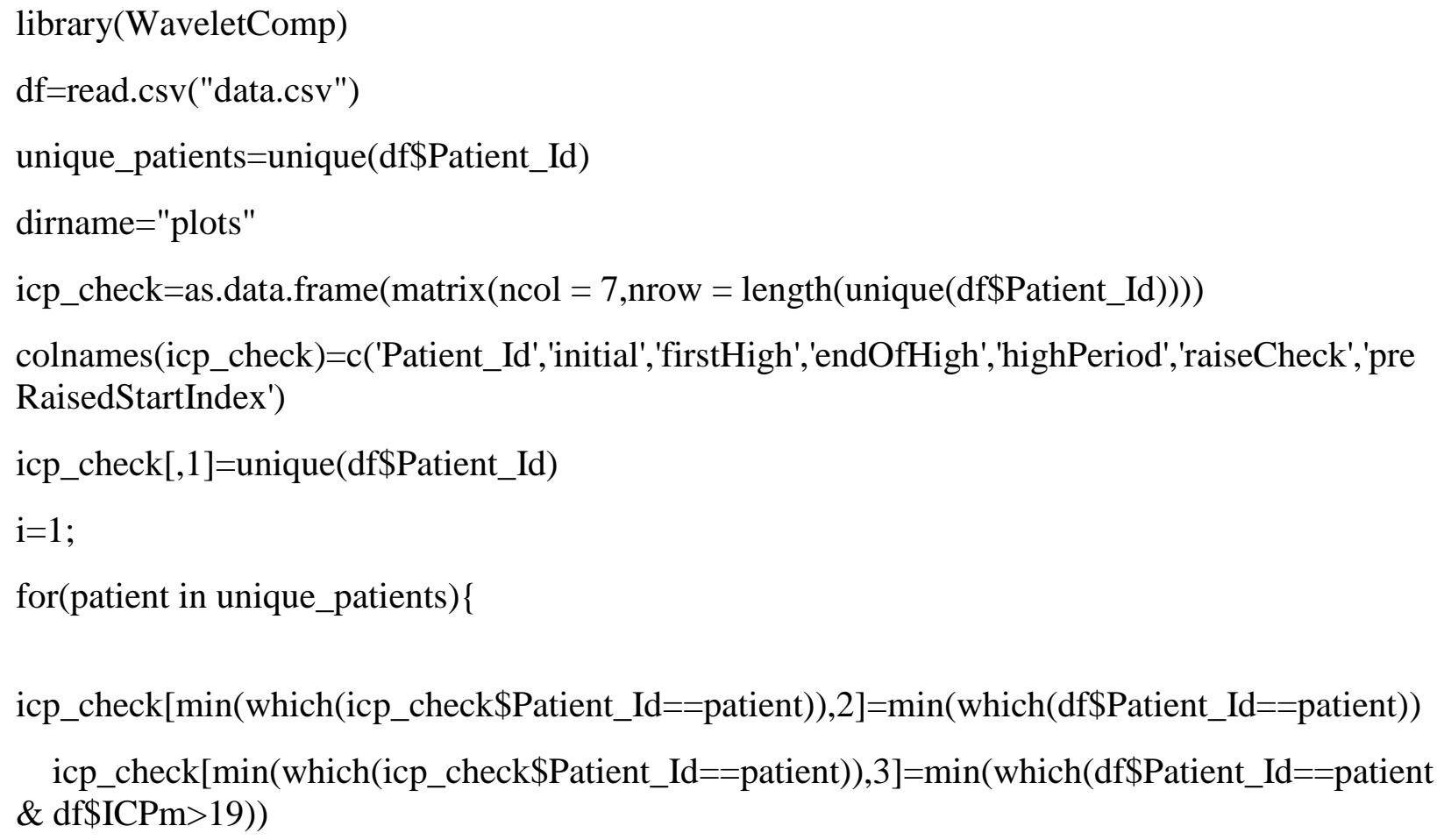


icp_check[min(which(icp_check\$Patient_Id==patient $)), 4]=\max ($ which $($ df $\$$ Patient_Id==patient $\&$ df $\$$ ICPm>19))

\}

icp_check[,5]=icp_check[,4]-icp_check[,3]

icp_check[,6]=ifelse(icp_check[,5]>1800,"raised","normal")

head(icp_check)

icp_check[,7]=icp_check[,3]-600

colnames(icp_check)=c('Patient_Id','initial','firstHigh','endOfHigh','highPeriod','raiseCheck','pre RaisedStartIndex')

head(icp_check)

impCol=colnames(df)[3:14]

normal_df=icp_check[icp_check\$raiseCheck=="normal",]

head(normal_df)

\section{\#Drawing normal plot}

dirType='normal';

for(patient in normal_df[,1])\{

dir.create(paste(dirname,toString(patient),sep="/"), showWarnings = "False")

dir.create(paste(dirname,toString(patient), dirType,sep="/"), showWarnings = "False")

\# dir.create(paste(dirname,dir))

for(col in impCol) \{

\# print(patient)

if(any(is.na(df[normal_df[normal_df\$Patient_Id==patient,]['initial'][,]:normal_df[normal_df\$Pat ient_Id==patient,]['firstHigh'][,],][col]))==TRUE) \{

\}

else

if(nrow(unique(df[normal_df[normal_df\$Patient_Id==patient,]['initial'][,]:normal_df[normal_df\$ Patient_Id==patient,]['firstHigh'][,],][col]))==1)\{ 


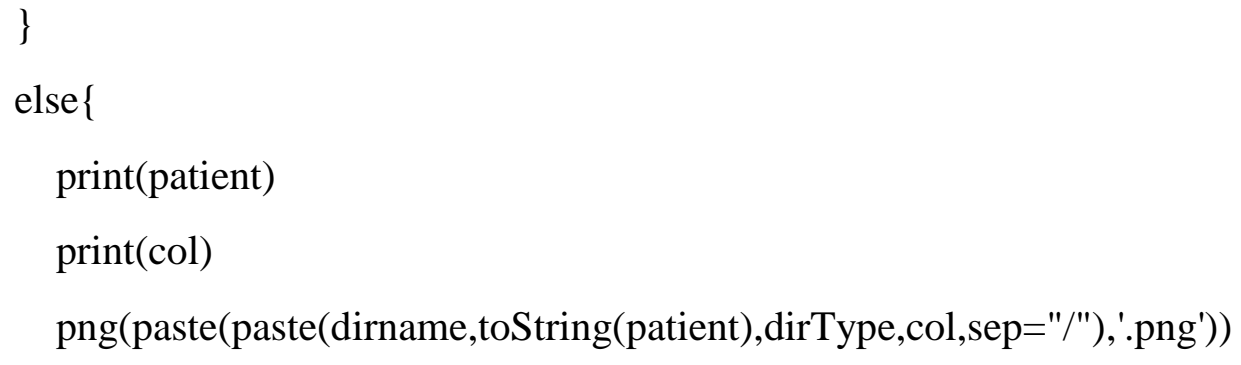

plot(df[normal_df[normal_df\$Patient_Id==patient,]['initial'][,]:normal_df[normal_df $\$$ Patient_Id ==patient,]['firstHigh'][,],][col][,],type='l',xlab="time",ylab="col")

dev.off()

png(paste(paste(dirname,toString(patient),dirType,col,sep="/"),'_wavelet.png'))

$\mathrm{x}=$ analyze.wavelet(df[normal_df[normal_df $\$$ Patient_Id==patient,]['initial'][,]:normal_df[normal _df\$Patient_Id==patient,]['firstHigh'][,],][col], n.sim $=1)$

$$
\text { loess } \cdot \operatorname{span}=0, \mathrm{dt}=1, \mathrm{dj}=1 / 10 \text {, lowerPeriod }=2 \text {, upperPeriod }=100 \text {, make. } p v a l=\mathrm{T} \text {, }
$$

wt.image $(x$, color.key = "interval", main = "wavelet power spectrum",

legend.params = list $($ lab = "wavelet power levels"),

$$
\text { periodlab }=\mathrm{col})
$$

dev.off()

\}

\}

\}

raised_df=icp_check[icp_check\$raiseCheck=='raised',]

\section{\#Drawing raised plot}

dirType='raised';

for(patient in raised_df[,1])\{ 
\# dir.create(paste(dirname,dir))

for(col in impCol) \{

print(patient)

if(any(is.na(df[raised_df[raised_df\$Patient_Id==patient,]['firstHigh'][,]:raised_df[raised_df\$Pati ent_Id==patient,]['endOfHigh'][,],][col]))==TRUE) \{

\}

else

if(nrow(unique(df[raised_df[raised_df \$Patient_Id==patient,]['firstHigh'][,]:raised_df[raised_df\$ Patient_Id==patient,]['endOfHigh'][,],][col]))==1)\{

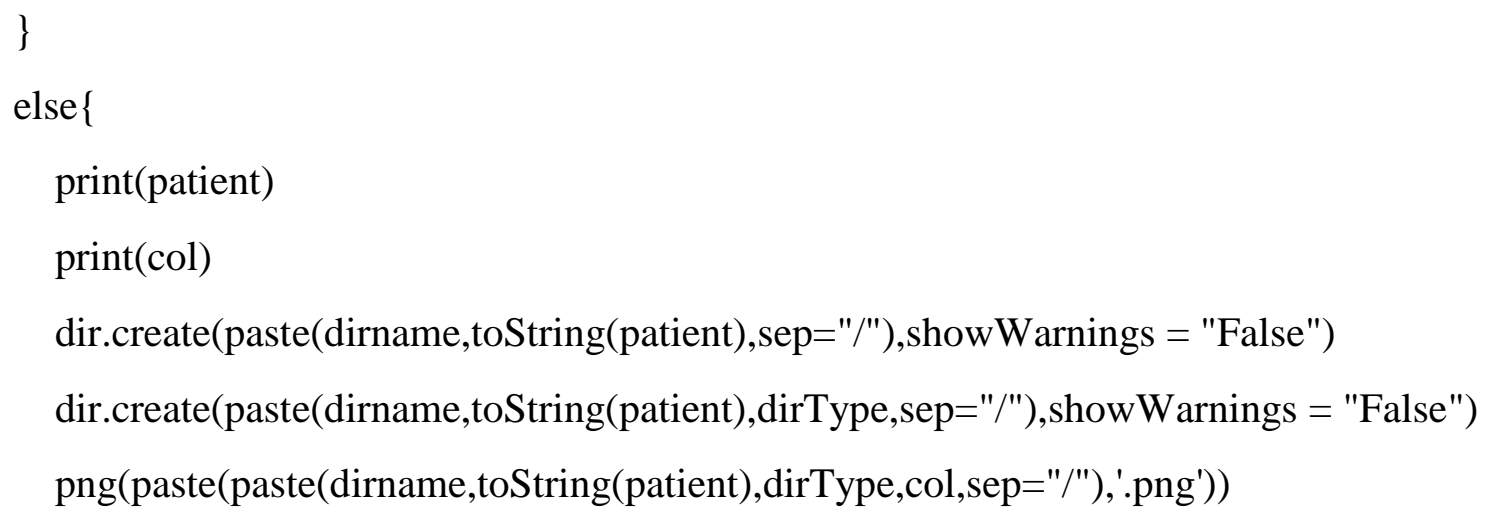

plot(df[raised_df[raised_df\$Patient_Id==patient,]['firstHigh'][,]:raised_df[raised_df\$Patient_Id= =patient,]['endOfHigh'][,],][col][,],type='l',xlab="time",ylab="col")

dev.off()

png(paste(paste(dirname,toString(patient),dirType,col,sep="/"),__wavelet.png'))

x=analyze.wavelet(df[raised_df[raised_df\$Patient_Id==patient,]['firstHigh'][,]:raised_df[raised_ df\$Patient_Id==patient,]['endOfHigh'][,],][col],

$$
\text { loess } \cdot \operatorname{span}=0, \mathrm{dt}=1, \mathrm{dj}=1 / 10, \text { lowerPeriod }=2, \quad \text { upperPeriod }=100,
$$
make.pval $=\mathrm{T}, \mathrm{n} \cdot \operatorname{sim}=1$ )

wt.image(x, color.key = "interval", main = "wavelet power spectrum",

legend.params $=\operatorname{list}(1 \mathrm{lab}=$ "wavelet power levels"),

periodlab $=\mathrm{col})$

dev.off() 


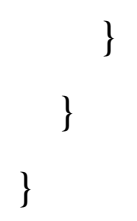

head(icp_check)

pre_raised_df=icp_check[icp_check $\$$ preRaisedStartIndex $>0$,]

head(pre_raised_df)

\section{\#Drawing pre-raised plot}

dirType='pre-raised';

for(patient in pre_raised_df[,1])\{

\# dir.create(paste(dirname,dir))

for(col in impCol) \{

print(patient)

if(any(is.na(df[pre_raised_df[pre_raised_df $\$ P a t i e n t \_I d==$ patient,]['preRaisedStartIndex'][,]:pre_r aised_df[pre_raised_df $\$$ Patient_Id==patient,]['firstHigh'][,],][col]))==TRUE)\{

\section{\}}

else

if(nrow(unique(df[pre_raised_df[pre_raised_df $\$$ Patient_Id==patient,]['preRaisedStartIndex'][,]:p re_raised_df[pre_raised_df $\$$ Patient_Id==patient,]['firstHigh'][,],][col]))==1)\{

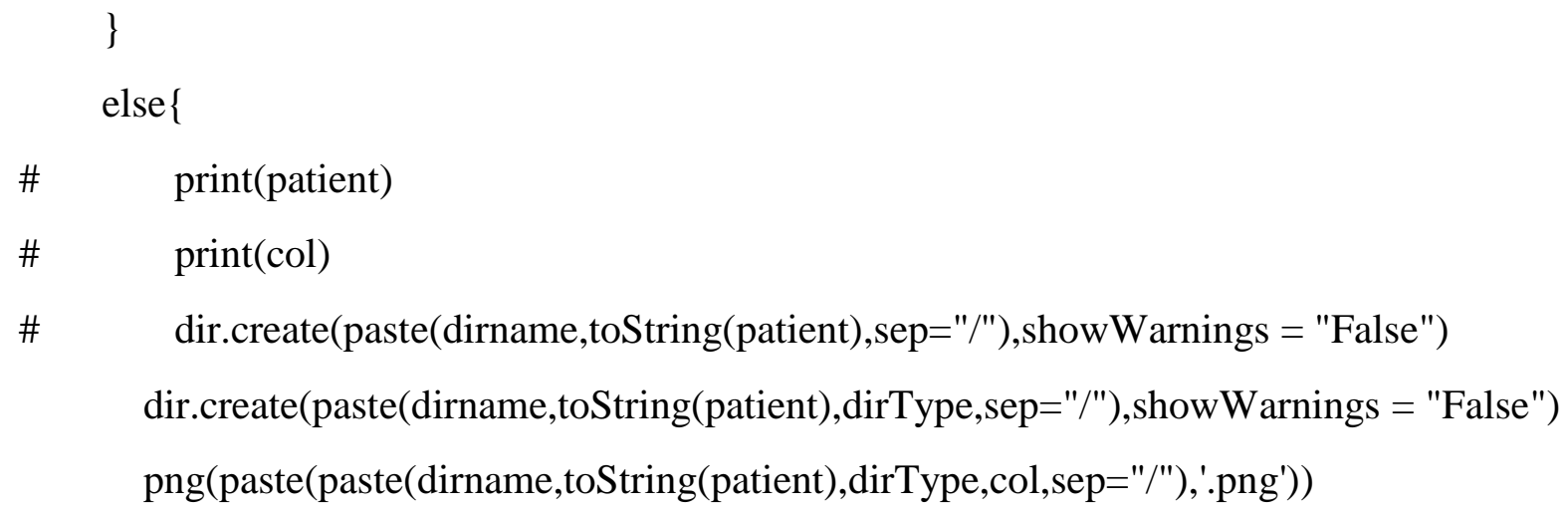


plot(df[pre_raised_df[pre_raised_df $\$$ Patient_Id==patient,]['preRaisedStartIndex'][,]:pre_raised_d f[pre_raised_df\$Patient_Id==patient,]['firstHigh'][,],][col][,],type='l',xlab="time",ylab="col")

dev.off()

png(paste(paste(dirname,toString(patient),dirType,col,sep="/"),'_wavelet.png'))

X=analyze.wavelet(df[pre_raised_df[pre_raised_df\$Patient_Id==patient,]['preRaisedStartIndex'][ ,]:pre_raised_df[pre_raised_df\$Patient_Id==patient,]['firstHigh'][,],][col], n. $\operatorname{sim}=1)$

loess. $\operatorname{span}=0, \mathrm{dt}=1, \mathrm{dj}=1 / 10$, lowerPeriod $=2$, upperPeriod $=100$, make.pval $=\mathrm{T}$, wt.image(x, color.key = "interval", main = "wavelet power spectrum",

legend.params = list(lab = "wavelet power levels"),

periodlab $=\mathrm{col})$

dev.off()

\}

\}

\}

Appendix J. Performance of the best trained model in MIMIC II Waveform database ( 2

hidden layers 200/150)

H2OBinomialMetrics: deep learning

MSE: 0.2135

RMSE: 0.46207

LogLoss: 2.2190

Mean Per-Class Error: 0.2168

AUC: 0.8627

Gini: 0.7254

Confusion Matrix (vertical: actual; across: predicted) for F1-optimal threshold:

\begin{tabular}{|c|c|c|c|c|}
\hline & 0 & 1 & Error & Rate \\
\hline 0 & 68764 & 27505 & 0.285 & 27504/96268 \\
\hline 1 & 9168 & 68763 & 0.117 & 9168/77931 \\
\hline Totals & 77932 & 96268 & 0.21 & $=36673 / 174200$ \\
\hline
\end{tabular}


Appendix K. Performance of the best trained model ( 2 hidden layers 200/150) on BrainIT database

H2OBinomialMetrics: deep learning

MSE: 0.05364

RMSE: 0.23160

LogLoss: 0.1905

Mean Per-Class Error: 0.0936

AUC: 0.9402

Gini: 0.8804

Confusion Matrix (vertical: actual; across: predicted) for F1-optimal threshold:

\begin{tabular}{llcllc} 
& 0 & 1 & Error & & Rate \\
0 & 4795 & 1050 & 0.179641 & $=$ & $1050 / 5845$ \\
1 & 223 & 28659 & 0.007721 & $=$ & $223 / 28882$ \\
\multicolumn{2}{l}{ Totals 5018} & 29709 & 0.036657 & $=$ & $1273 / 34727$
\end{tabular}

\section{Appendix L. Multi-Roc plot}

True Positive Rate vs False Positive Rate

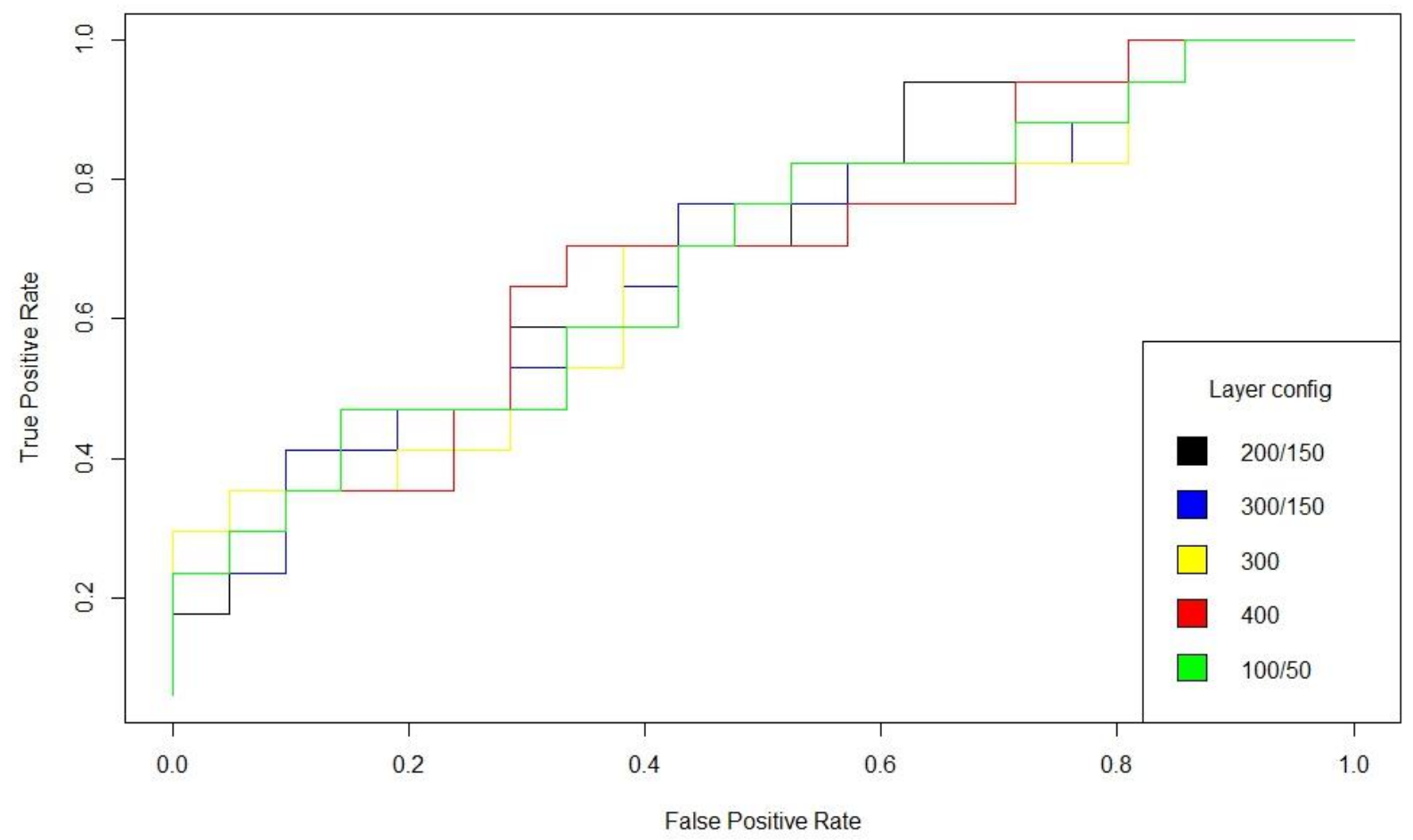




\section{References}

[1] A. I. Maas, N. Stocchetti, and R. Bullock, "Moderate and severe traumatic brain injury in adults," Lancet Neurol., vol. 7, no. 8, pp. 728-741, Aug. 2008.

[2] M. Hüser, "Forecasting intracranial hypertension using time series and waveform features," ETH Zürich, 2015.

[3] Z. Feldman et al., "Effect of head elevation on intracranial pressure, cerebral perfusion pressure, and cerebral blood flow in head-injured patients," J. Neurosurg., vol. 76, no. 2, pp. 207-211, 1992.

[4] M. J. Rosner and D. P. Becker, "ICP monitoring: complications and associated factors.," Clin. Neurosurg., vol. 23, pp. 494-519, 1976.

[5] M. Hüser, V. De Luca, M. Jaggi, W. Karlen, and E. Keller, "Forecasting intracranial hypertension using waveform and time series features," in Vasospasm 2015-13th International Conference on Neurovascular Events after Subarachnoid Hemorrhage, 2015.

[6] O. L. Cremer et al., "Effect of intracranial pressure monitoring and targeted intensive care on functional outcome after severe head injury," Crit. Care Med., vol. 33, no. 10, pp. 22072213, 2005.

[7] A. T. Blei, S. Olafsson, S. Webster, and R. Levy, "Complications of intracranial pressure monitoring in fulminant hepatic failure," Lancet, vol. 341, no. 8838, pp. 157-158, 1993.

[8] S. A. Gauri et al., "Surgical outcome of intraventricular hemorrhage using an intraventricular catheter and its complications at Civil," Rawal Med. J., vol. 42, no. 1, 2017.

[9] P. H. Raboel, J. Bartek, M. Andresen, B. M. Bellander, and B. Romner, "Intracranial pressure monitoring: invasive versus non-invasive methods a review," Crit. Care Res. Pract., vol. 2012, 2012.

[10] A. Di Ieva, E. M. Schmitz, and M. D. Cusimano, “Analysis of intracranial pressure: past, present, and future," Neurosci., vol. 19, no. 6, pp. 592-603, 2013.

[11] M. S. Greenberg and N. Arredondo, "Handbook of neurosurgery," 2006. 
[12] S. Cecil, P. M. Chen, S. E. Callaway, S. M. Rowland, D. E. Adler, and J. W. Chen, "Traumatic brain injury advanced multimodal neuromonitoring from theory to clinical practice," Crit. Care Nurse, vol. 31, no. 2, pp. 25-37, 2011.

[13] J. P. Berdahl, M. P. Fautsch, S. S. Stinnett, and R. R. Allingham, "Intracranial pressure in primary open angle glaucoma, normal tension glaucoma, and ocular hypertension: a case-control study," Invest. Ophthalmol. Vis. Sci., vol. 49, no. 12, pp. 5412-5418, 2008.

[14] N. Carney et al., "Guidelines for the management of severe traumatic brain injury," Neurosurgery, vol. 80, no. 1, pp. 6-15, 2017.

[15] K. Welch, “The intracranial pressure in infants," J. Neurosurg., vol. 52, no. 5, pp. 693-699, 1980.

[16] N. Juul, G. F. Morris, S. B. Marshall, E. C. of the International Selfotel Trial, and L. F. Marshall, "Intracranial hypertension and cerebral perfusion pressure: influence on neurological deterioration and outcome in severe head injury," J. Neurosurg., vol. 92, no. 1, pp. 1-6, 2000.

[17] R. Bullock et al., "Guidelines for the management of severe head injury," Eur. J. Emerg. Med., vol. 3, pp. 109-127, 1996.

[18] S. I. Stiver, "Complications of decompressive craniectomy for traumatic brain injury," Neurosurg. Focus, vol. 26, no. 6, p. E7, 2009.

[19] D. Laskowitz and G. Grant, Translational research in traumatic brain injury, vol. 57. CRC Press, 2016.

[20] D. T. Cross, C. J. Moran, E. E. Angtuaco, J. M. Milburn, M. N. Diringer, and R. G. Dacey, "Intracranial pressure monitoring during intraarterial papaverine infusion for cerebral vasospasm.," Am. J. Neuroradiol., vol. 19, no. 7, pp. 1319-1323, 1998.

[21] C. Wiegand and P. Richards, "Measurement of intracranial pressure in children: a critical review of current methods," Dev. Med. Child Neurol., vol. 49, no. 12, pp. 935-941, 2007.

[22] M. Lyon et al., "Effect of History of Mild Traumatic Brain Injury on Optic Nerve Sheath Diameter Changes after Valsalva Maneuver," J. Neurotrauma, 2018. 
[23] M. Haredy, G. Zuccoli, M. Tamber, A. Davis, K. Nischal, and J. A. Goldstein, "Use of neuroimaging measurements of optic nerve sheath diameter to assess intracranial pressure in craniosynostosis," Child's Nerv. Syst., vol. 34, no. 5, pp. 939-946, 2018.

[24] E. J. Kim, B.-N. Koo, S. H. Choi, K. Park, and M.-S. Kim, "Ultrasonographic optic nerve sheath diameter for predicting elevated intracranial pressure during laparoscopic surgery: a systematic review and meta-analysis," Surg. Endosc., vol. 32, no. 1, pp. 175-182, 2018.

[25] I.-J. Jun et al., "Effect of Mannitol on Ultrasonographically Measured Optic Nerve Sheath Diameter as a Surrogate for Intracranial Pressure During Robot-Assisted Laparoscopic Prostatectomy with Pneumoperitoneum and the Trendelenburg Position," J. Endourol., 2018.

[26] H. Diaz, "Optic nerve sheath diameter for diagnosing increased intracranial pressure in clinical practice.," 2018.

[27] S. K. Singh and K. Bhatia, "Ultrasonographic optic nerve sheath diameter as a surrogate measure of raised intracranial pressure in severe pregnancy-induced hypertension patients," Anesth. essays Res., vol. 12, no. 1, p. 42, 2018.

[28] J. B. Rosenberg, A. L. Shiloh, R. H. Savel, and L. A. Eisen, "Non-invasive methods of estimating intracranial pressure," Neurocrit. Care, vol. 15, no. 3, pp. 599-608, 2011.

[29] D. Low, V. Kuralmani, S. K. Ng, K. K. Lee, I. Ng, and B. T. Ang, "Prediction of outcome utilizing both physiological and biochemical parameters in severe head injury," $J$. Neurotrauma, vol. 26, no. 8, pp. 1177-1182, 2009.

[30] A. Bravi, A. Longtin, and A. J. E. Seely, "Review and classification of variability analysis techniques with clinical applications," Biomed. Eng. Online, vol. 10, no. 1, p. 90, 2011.

[31] S. H. Strogatz, Nonlinear dynamics and chaos: with applications to physics, biology, chemistry, and engineering. CRC Press, 2018.

[32] T. Schäck, M. Muma, M. Feng, C. Guan, and A. M. Zoubir, "Robust Nonlinear Causality Analysis of Nonstationary Multivariate Physiological Time Series," IEEE Trans. Biomed. Eng., vol. 65, no. 6, pp. 1213-1225, 2018. 
[33] E. G. Tolkacheva, B. Feeny, and X. Zhao, "Special Issue: Nonlinear and Computational Dynamics in Biomedical Applications," J. Comput. Nonlinear Dyn., vol. 13, no. 6, p. 68001, 2018.

[34] S. Kim, F. Scalzo, M. Bergsneider, P. Vespa, N. Martin, and X. Hu, "Noninvasive intracranial pressure assessment based on a data-mining approach using a nonlinear mapping function,” IEEE Trans. Biomed. Eng., vol. 59, no. 3, pp. 619-626, 2012.

[35] P. K. Narotam, J. F. Morrison, M. D. Schmidt, and N. Nathoo, "Physiological complexity of acute traumatic brain injury in patients treated with a brain oxygen protocol: Utility of symbolic regression in predictive modeling of a dynamical system," J. Neurotrauma, vol. 31, no. 7, pp. 630-641, 2014.

[36] G. V Varsos et al., "A noninvasive estimation of cerebral perfusion pressure using critical closing pressure," J. Neurosurg., vol. 123, no. 3, pp. 638-648, 2015.

[37] J. A. Hughes, E. C. Jackson, and M. Daley, "Modelling intracranial pressure with noninvasive physiological measures," in Computational Intelligence in Bioinformatics and Computational Biology (CIBCB), 2017 IEEE Conference on, 2017, pp. 1-8.

[38] S. Neff and R. P. Subramaniam, "Monro-Kellie doctrine.," J. Neurosurg., vol. 85, no. 6, p. $1195,1996$.

[39] A. Monro, "Observations on the Structure and Functions of the Nervous System," 1783.

[40] L. A. Steiner and P. J. D. Andrews, "Monitoring the injured brain: ICP and CBF," Br. J. Anaesth., vol. 97, no. 1, pp. 26-38, 2006.

[41] A. J. E. Seely and P. T. Macklem, "Complex systems and the technology of variability analysis," Crit. care, vol. 8, no. 6, p. R367, 2004.

[42] K. H. O Phelan et al., "Patterns of increased intracranial pressure after severe traumatic brain injury," Neurocrit. Care, vol. 10, no. 3, p. 280, 2009.

[43] A. Bellotti, A. Rapana, C. Iaccarino, and M. Schonauer, "Intracranial pressure monitoring after endoscopic third ventriculostomy: an effective method to manage the adaptation period," Clin. Neurol. Neurosurg., vol. 103, no. 4, pp. 223-227, 2001. 
[44] P. K. Eide, "A new method for processing of continuous intracranial pressure signals," Med. Eng. Phys., vol. 28, no. 6, pp. 579-587, 2006.

[45] S. A. Mayer and J. Y. Chong, "Critical care management of increased intracranial pressure," J. Intensive Care Med., vol. 17, no. 2, pp. 55-67, 2002.

[46] D. Santamarta, R. Hornero, D. Abásolo, M. Mart'linez-Madrigal, J. Fernández, and J. Garc'lia-Cosamalón, "Complexity analysis of the cerebrospinal fluid pulse waveform during infusion studies," Child's Nerv. Syst., vol. 26, no. 12, pp. 1683-1689, 2010.

[47] P. K. Eide, A. Sorteberg, G. Bentsen, P. B. Marthinsen, A. Stubhaug, and W. Sorteberg, "Pressure-derived versus pressure wave amplitude--derived indices of cerebrovascular pressure reactivity in relation to early clinical state and 12-month outcome following aneurysmal subarachnoid hemorrhage," J. Neurosurg., vol. 116, no. 5, pp. 961-971, 2012.

[48] S. Holm and P. K. Eide, "The frequency domain versus time domain methods for processing of intracranial pressure (ICP) signals," Med. Eng. Phys., vol. 30, no. 2, pp. 164-170, 2008.

[49] J. Klingelhöfer, B. Conrad, R. Benecke, D. Sander, and E. Markakis, "Evaluation of intracranial pressure from transcranial Doppler studies in cerebral disease," J. Neurol., vol. 235, no. 3, pp. 159-162, 1988.

[50] J. Szewczykowski et al., "A method of estimating intracranial decompensation in man," $J$. Neurosurg., vol. 45, no. 2, pp. 155-158, 1976.

[51] F.-C. Tsui, M. Sun, C.-C. Li, and R. J. Sclabassi, "A wavelet based neural network for prediction of ICP signal," in Engineering in Medicine and Biology Society, 1995., IEEE 17th Annual Conference, 1995, vol. 2, pp. 1045-1046.

[52] M. Barth et al., "Correlation of clinical outcome with pressure-, oxygen-, and flow-related indices of cerebrovascular reactivity in patients following aneurysmal SAH," Neurocrit. Care, vol. 12, no. 2, pp. 234-243, 2010.

[53] I. Bajla, R. Škoviera, and M. Teplan, "An alternative of the sliding window approach in time series clustering of intracranial pressure for patients with traumatic brain injury," in Measurement, 2017 11th International Conference on, 2017, pp. 47-50. 
[54] P. C. Whitfield, P. J. Kirkpatrick, M. Czosnyka, and J. D. Pickard, "Management of severe traumatic brain injury by decompressive craniectomy," Neurosurgery, vol. 49, no. 1, pp. 225-226, 2001.

[55] J. Szewczykowski, S. liwka, A. Kunicki, P. Dytko, and J. Korsak-liwka, "A fast method of estimating the elastance of the intracranial system: A practical application in neurosurgery," J. Neurosurg., vol. 47, no. 1, pp. 19-26, 1977.

[56] J.-Y. Fan, C. Kirkness, P. Vicini, R. Burr, and P. Mitchell, "An approach to determining intracranial pressure variability capable of predicting decreased intracranial adaptive capacity in patients with traumatic brain injury," Biol. Res. Nurs., vol. 11, no. 4, pp. $317-$ $324,2010$.

[57] M. Czosnyka and J. D. Pickard, "Monitoring and interpretation of intracranial pressure," J. Neurol. Neurosurg. Psychiatry, vol. 75, no. 6, pp. 813-821, 2004.

[58] O. B. Paulson, S. Strandgaard, and L. Edvinsson, "Cerebral autoregulation.," Cerebrovasc. Brain Metab. Rev., vol. 2, no. 2, pp. 161-192, 1990.

[59] A. S. Weigend, Time series prediction: forecasting the future and understanding the past. Routledge, 2018.

[60] S. M. Pincus, I. M. Gladstone, and R. A. Ehrenkranz, "A regularity statistic for medical data analysis," J. Clin. Monit., vol. 7, no. 4, pp. 335-345, 1991.

[61] R. Hornero, M. Aboy, D. Abásolo, J. McNames, and B. Goldstein, "Interpretation of approximate entropy: analysis of intracranial pressure approximate entropy during acute intracranial hypertension," IEEE Trans. Biomed. Eng., vol. 52, no. 10, pp. 1671-1680, 2005.

[62] R. Hornero, M. Aboy, and D. Abásolo, "Analysis of intracranial pressure during acute intracranial hypertension using Lempel--Ziv complexity: further evidence," Med. Biol. Eng. Comput., vol. 45, no. 6, pp. 617-620, 2007.

[63] M. Aboy, R. Hornero, D. Abásolo, and D. Álvarez, "Interpretation of the Lempel-Ziv complexity measure in the context of biomedical signal analysis," IEEE Trans. Biomed. Eng., vol. 53, no. 11, pp. 2282-2288, 2006. 
[64] A. Lempel and J. Ziv, "On the complexity of finite sequences IEEE Transactions on Information Theory Vol IT22." 1976.

[65] N. Lundberg, "Continuous recording and control of ventricular fluid pressure in neurosurgical practice.," Acta Psychiatr. Scand. Suppl., vol. 36, no. 149, p. 1, 1960.

[66] G. Castellani et al., "Plateau waves in head injured patients requiring neurocritical care," Neurocrit. Care, vol. 11, no. 2, pp. 143-150, 2009.

[67] M. H. Morgalla, F. Stumm, and G. Hesse, "A computer-based method for continuous single pulse analysis of intracranial pressure waves," J. Neurol. Sci., vol. 168, no. 2, pp. 90-95, 1999.

[68] H. E. Heissler, K. König, J. K. Krauss, and E. Rickels, "Analysis of intracranial pressure time series using wavelets (Haar basis functions)," in Intracranial Pressure and Brain Monitoring XIV, Springer, 2012, pp. 87-91.

[69] C. B. Shields, P. C. McGraw, and H. D. Garretson, "Accurate intracranial pressure monitoring," Neurosurgery, vol. 14, no. 5, pp. 592-593, 1984.

[70] R. Hamilton et al., "Forecasting intracranial pressure elevation using pulse waveform morphology," in Engineering in Medicine and Biology Society, 2009. EMBC 2009. Annual International Conference of the IEEE, 2009, pp. 4331-4334.

[71] X. Hu, P. Xu, F. Scalzo, P. Vespa, and M. Bergsneider, "Morphological clustering and analysis of continuous intracranial pressure," IEEE Trans. Biomed. Eng., vol. 56, no. 3, pp. 696-705, 2009.

[72] F. Scalzo, M. Bergsneider, P. M. Vespa, N. A. Martin, and X. Hu, "Intracranial pressure signal morphology: real-time tracking," IEEE Pulse, vol. 3, no. 2, pp. 49-52, 2012.

[73] F. M. Kashif, G. C. Verghese, V. Novak, M. Czosnyka, and T. Heldt, "Model-based noninvasive estimation of intracranial pressure from cerebral blood flow velocity and arterial pressure," Sci. Transl. Med., vol. 4, no. 129, p. 129ra44--129ra44, 2012.

[74] D. E. Newland, "Wavelet analysis of vibration: part 2 wavelet maps," J. Vib. Acoust., vol. 116, no. 4, pp. 417-425, 1994. 
[75] B. C. Lam, "Challenges to Time Series Analysis in the Computer Age," Stat. your Futur. power, pp. 1-15, 2014.

[76] J. S. Bendat and A. G. Piersol, "Engineering applications of correlation and spectral analysis," New York, Wiley-Interscience, 1980. 315 p., 1980.

[77] S. M. Kay and S. L. Marple, "Spectrum analysis a modern perspective," Proc. IEEE, vol. 69, no. 11, pp. 1380-1419, 1981.

[78] J. Bohata, M. Komanec, J. Spáčil, Z. Ghassemlooy, S. Zvánovec, and R. Slav’lik, “24--26 GHz radio-over-fiber and free-space optics for fifth-generation systems," Opt. Lett., vol. 43, no. 5, pp. 1035-1038, 2018.

[79] D. C. Robertson, O. I. Camps, J. S. Mayer, and W. B. Gish, "Wavelets and electromagnetic power system transients," IEEE Trans. Power Deliv., vol. 11, no. 2, pp. 1050-1058, 1996.

[80] R. Lopes and N. Betrouni, "Fractal and multifractal analysis: a review," Med. Image Anal., vol. 13, no. 4, pp. 634-649, 2009.

[81] A. L. Goldberger, "Non-linear dynamics for clinicians: chaos theory, fractals, and complexity at the bedside," Lancet, vol. 347, no. 9011, pp. 1312-1314, 1996.

[82] R. L. Burr, C. J. Kirkness, P. H. Mitchell, and others, "Detrended fluctuation analysis of intracranial pressure predicts outcome following traumatic brain injury," IEEE Trans. Biomed. Eng., vol. 55, no. 11, pp. 2509-2518, 2008.

[83] A. L. Goldberger, L. A. N. Amaral, J. M. Hausdorff, P. C. Ivanov, C.-K. Peng, and H. E. Stanley, "Fractal dynamics in physiology: alterations with disease and aging," Proc. Natl. Acad. Sci., vol. 99, no. suppl 1, pp. 2466-2472, 2002.

[84] B. Schuster-Böckler and A. Bateman, "An introduction to hidden Markov models," Curr. Protoc. Bioinforma., vol. 18, no. 1, p. A--3A, 2007.

[85] K. Seymore, A. McCallum, and R. Rosenfeld, "Learning hidden Markov model structure for information extraction," in AAAI-99 workshop on machine learning for information extraction, 1999, pp. 37-42.

[86] I. Rezek and S. J. Roberts, "Ensemble hidden markov models for biosignal analysis," in 
Digital Signal Processing, 2002. DSP 2002. 2002 14th International Conference on, 2002, vol. 1, pp. 387-391.

[87] D. Novák, L. Lhotská, D. Cuesta-Frau, M. Aboy, and B. Goldstein, Clustering of intracranial pressure using hidden Markov models. na, 2004.

[88] M. I. Jordan, Z. Ghahramani, and L. K. Saul, "Hidden Markov decision trees," in Advances in neural information processing systems, 1997, pp. 501-507.

[89] L. Kleeman, "Understanding and applying Kalman filtering," in Proceedings of the Second Workshop on Perceptive Systems, Curtin University of Technology, Perth Western Australia (25-26 January 1996), 1996.

[90] H. W. Sorenson, "Least-squares estimation: from Gauss to Kalman," IEEE Spectr., vol. 7, no. 7, pp. 63-68, 1970.

[91] S. W. Smith and others, "The scientist and engineer's guide to digital signal processing," 1997.

[92] F. Daum, "Nonlinear filters: beyond the Kalman filter," IEEE Aerosp. Electron. Syst. Mag., vol. 20, no. 8, pp. 57-69, 2005.

[93] J. McNames and M. Aboy, "Statistical modeling of cardiovascular signals and parameter estimation based on the extended Kalman filter," IEEE Trans. Biomed. Eng., vol. 55, no. 1, pp. 119-129, 2008.

[94] Y. S. Shmaliy, Y. Neuvo, and S. Khan, "Review of Unbiased FIR Filters, Smoothers, and Predictors for Polynomial Signals," 2018.

[95] F. Ma, F. Liu, X. Zhang, P. Wang, H. Bai, and H. Guo, “An ultrasonic positioning algorithm based on maximum correntropy criterion extended Kalman filter weighted centroid," Signal, Image Video Process., pp. 1-9, 2018.

[96] X. Hu, V. Nenov, M. Bergsneider, T. C. Glenn, P. Vespa, and N. Martin, "Estimation of hidden state variables of the intracranial system using constrained nonlinear Kalman filters," IEEE Trans. Biomed. Eng., vol. 54, no. 4, pp. 597-610, 2007.

[97] M. Aboy, O. W. Márquez, J. McNames, R. Hornero, T. Trong, and B. Goldstein, “Adaptive 
modeling and spectral estimation of nonstationary biomedical signals based on Kalman filtering," IEEE Trans. Biomed. Eng., vol. 52, no. 8, pp. 1485-1489, 2005.

[98] E. A. Wan and A. T. Nelson, "Neural dual extended Kalman filtering: applications in speech enhancement and monaural blind signal separation," in Neural Networks for Signal Processing [1997] VII. Proceedings of the 1997 IEEE Workshop, 1997, pp. 466-475.

[99] S. B. Kotsiantis, I. Zaharakis, and P. Pintelas, "Supervised machine learning: A review of classification techniques," Emerg. Artif. Intell. Appl. Comput. Eng., vol. 160, pp. 3-24, 2007.

[100] S. Dreiseitl and L. Ohno-Machado, "Logistic regression and artificial neural network classification models: a methodology review," J. Biomed. Inform., vol. 35, no. 5-6, pp. 352359, 2002.

[101] J.-S. Shieh, C.-F. Chou, S.-J. Huang, and M.-C. Kao, "Intracranial pressure model in intensive care unit using a simple recurrent neural network through time," Neurocomputing, vol. 57, pp. 239-256, 2004.

[102] C. J. Willmott, “On the validation of models," Phys. Geogr., vol. 2, no. 2, pp. 184-194, 1981.

[103] M. A. Babyak, "What you see may not be what you get: a brief, nontechnical introduction to overfitting in regression-type models," Psychosom. Med., vol. 66, no. 3, pp. 411-421, 2004.

[104] F. E. Harrell, "Ordinal logistic regression," in Regression modeling strategies, Springer, 2001, pp. 331-343.

[105] R. Gassner, T. Tuli, O. Hächl, A. Rudisch, and H. Ulmer, "Cranio-maxillofacial trauma: a 10 year review of 9543 cases with 21067 injuries," J. cranio-maxillo-facial Surg., vol. 31, no. 1, pp. 51-61, 2003.

[106] M. Swiercz, Z. Mariak, J. Krejza, J. Lewko, and P. Szydlik, "Intracranial pressure processing with artificial neural networks: prediction of ICP trends," Acta Neurochir. (Wien)., vol. 142, no. 4, pp. 401-406, 2000. 
[107] A. Fanelli, F. W. Vonberg, R. Jaishankar, S. M. Imaduddin, R. C. Tasker, and T. Heldt, "Regression-based noninvasive estimation of intracranial pressure," in Engineering in Medicine and Biology Society (EMBC), 2017 39th Annual International Conference of the IEEE, 2017, pp. 4001-4004.

[108] F. Güiza, B. Depreitere, I. Piper, G. den Berghe, and G. Meyfroidt, "Novel methods to predict increased intracranial pressure during intensive care and long-term neurologic outcome after traumatic brain injury: development and validation in a multicenter dataset," Crit. Care Med., vol. 41, no. 2, pp. 554-564, 2013.

[109] J. V Tu, “Advantages and disadvantages of using artificial neural networks versus logistic regression for predicting medical outcomes," J. Clin. Epidemiol., vol. 49, no. 11, pp. 1225$1231,1996$.

[110] V. Cherkassky and Y. Ma, "Practical selection of SVM parameters and noise estimation for SVM regression," Neural networks, vol. 17, no. 1, pp. 113-126, 2004.

[111] V. Vapnik, The nature of statistical learning theory. Springer science \& business media, 2013.

[112] B. Scholkopf and A. J. Smola, Learning with kernels: support vector machines, regularization, optimization, and beyond. MIT press, 2001.

[113] K. Fukunaga, Introduction to statistical pattern recognition. Academic press, 2013.

[114] S. Abe, Support vector machines for pattern classification, vol. 2. Springer, 2005.

[115] T. Evgeniou and M. Pontil, "Regularized multi--task learning," in Proceedings of the tenth ACM SIGKDD international conference on Knowledge discovery and data mining, 2004, pp. 109-117.

[116] H. Bhavsar and M. H. Panchal, "A review on support vector machine for data classification," Int. J. Adv. Res. Comput. Eng. Technol., vol. 1, no. 10, p. pp--185, 2012.

[117] W. Chen, C. H. Cockrell, K. Ward, and K. Najarian, "Predictability of intracranial pressure level in traumatic brain injury: features extraction, statistical analysis and machine learningbased evaluation,” Int. J. Data Min. Bioinform., vol. 8, no. 4, pp. 480-494, 2013. 
[118] J. Kazmierska and J. Malicki, “Application of the $\mathrm{Na}\{" \mathrm{l} i\}$ ve Bayesian Classifier to optimize treatment decisions," Radiother. Oncol., vol. 86, no. 2, pp. 211-216, 2008.

[119] W. Klement, S. Wilk, W. Michalowski, K. J. Farion, M. H. Osmond, and V. Verter, "Predicting the need for $\mathrm{CT}$ imaging in children with minor head injury using an ensemble of Naive Bayes classifiers," Artif. Intell. Med., vol. 54, no. 3, pp. 163-170, 2012.

[120] W. Wei, S. Visweswaran, and G. F. Cooper, "The application of naive Bayes model averaging to predict Alzheimer's disease from genome-wide data," J. Am. Med. Informatics Assoc., vol. 18, no. 4, pp. 370-375, 2011.

[121] D. D. Lewis, "Naive (Bayes) at forty: The independence assumption in information retrieval," in European conference on machine learning, 1998, pp. 4-15.

[122] F. Scalzo, R. Hamilton, S. Asgari, S. Kim, and X. Hu, "Intracranial hypertension prediction using extremely randomized decision trees," Med. Eng. Phys., vol. 34, no. 8, pp. 1058$1065,2012$.

[123] M. Galeano et al., "Classification of morphological features extracted from intracranial pressure recordings in the diagnosis of normal pressure hydrocephalus (NPH), " in Engineering in Medicine and Biology Society, EMBC, 2011 Annual International Conference of the IEEE, 2011, pp. 2768-2771.

[124] J. R. Quinlan, "Simplifying decision trees," 1986.

[125] R. T. Clemen and T. Reilly, Making hard decisions with DecisionTools. Cengage Learning, 2013.

[126] Z. K. Peng and F. L. Chu, "Application of the wavelet transform in machine condition monitoring and fault diagnostics: a review with bibliography," Mech. Syst. Signal Process., vol. 18, no. 2, pp. 199-221, 2004.

[127] R. Q. Quiroga, Z. Nadasdy, and Y. Ben-Shaul, "Unsupervised Spike Detection and Sorting with Wavelets and Superparamagnetic Clustering," Neural Comput., vol. 16, no. 8, pp. 1661-1687, 2004.

[128] S. Ghosh-Dastidar and H. Adeli, "Wavelet-Clustering-Neural Network Model for Freeway 
Incident Detection," Comput. Civ. Infrastruct. Eng., vol. 18, no. 5, pp. 325-338, 2003.

[129] G. Sheikholeslami, S. Chatterjee, and A. Zhang, "WaveCluster: A Wavelet-based Clustering Approach for Spatial Data in Very Large Databases," VLDB J., vol. 8, no. 3-4, pp. 289-304, Feb. 2000.

[130] M. Vlachos, J. Lin, E. Keogh, and D. Gunopulos, "A wavelet-based anytime algorithm for k-means clustering of time series," in In Proc. Workshop on Clustering High Dimensionality Data and Its Applications, 2003.

[131] A. B. Geva and D. H. Kerem, "Forecasting generalized epileptic seizures from the EEG signal by wavelet analysis and dynamic unsupervised fuzzy clustering," IEEE Trans. Biomed. Eng., vol. 45, no. 10, pp. 1205-1216, 1998.

[132] M. Murugappan, M. Rizon, R. Nagarajan, S. Yaacob, D. Hazry, and I. Zunaidi, "Timefrequency analysis of EEG signals for human emotion detection," in 4th Kuala Lumpur International Conference on Biomedical Engineering 2008, 2008, pp. 262-265.

[133] Q. Xu, T. L. Nwe, and C. Guan, "Cluster-based analysis for personalized stress evaluation using physiological signals," IEEE J. Biomed. Heal. informatics, vol. 19, no. 1, pp. 275281, 2015.

[134] O. Faust, U. R. Acharya, H. Adeli, and A. Adeli, "Wavelet-based EEG processing for computer-aided seizure detection and epilepsy diagnosis," Seizure, vol. 26, pp. 56-64, 2015.

[135] W. W. Melek, Z. Lu, A. Kapps, and W. D. Fraser, "Comparison of trend detection algorithms in the analysis of physiological time-series data," IEEE Trans. Biomed. Eng., vol. 52, no. 4, pp. 639-651, 2005.

[136] B. N. Li, M. C. Dong, and M. I. Vai, "Modelling cardiovascular physiological signals using adaptive Hermite and wavelet basis functions," IET signal Process., vol. 4, no. 5, pp. 588$597,2010$.

[137] A. J. Schwarz, B. Whitcher, A. Gozzi, T. Reese, and A. Bifone, "Study-level wavelet cluster analysis and data-driven signal models in pharmacological MRI," J. Neurosci. Methods, vol. 159, no. 2, pp. 346-360, 2007. 
[138] C.-F. Chao, J.-A. Jiang, M.-J. Chiu, and R.-G. Lee, "Wavelet-based processing and adaptive fuzzy clustering for automated long-term polysomnography analysis," in Acoustics, Speech and Signal Processing, 2006. ICASSP 2006 Proceedings. 2006 IEEE International Conference on, 2006, vol. 2, pp. II--II.

[139] M. Saeed et al., "Multiparameter Intelligent Monitoring in Intensive Care II (MIMIC-II): a public-access intensive care unit database," Crit. Care Med., vol. 39, no. 5, p. 952, 2011.

[140] I. Piper et al., "The BrainIT group: concept and core dataset definition," Acta Neurochir. (Wien)., vol. 145, no. 8, pp. 615-629, 2003.

[141] A. Rajagopal, R. B. Hamilton, and F. Scalzo, "Noise reduction in intracranial pressure signal using causal shape manifolds," Biomed. Signal Process. Control, vol. 28, pp. 19-26, 2016.

[142] P. Royston and others, "Multiple imputation of missing values," Stata J., vol. 4, no. 3, pp. 227-241, 2004.

[143] I. B. M. S. Statistics, “version 22,” IBM New York, NY, USA, 2013.

[144] I. Kononenko, "Estimating attributes: analysis and extensions of RELIEF," in European conference on machine learning, 1994, pp. 171-182.

[145] RStudio Team, “RStudio: Integrated Development Environment for R.” Boston, MA, 2016.

[146] J.-O. Kim and C. W. Mueller, Factor analysis: Statistical methods and practical issues, vol. 14. Sage, 1978.

[147] A. E. Hurley et al., "Exploratory and confirmatory factor analysis: Guidelines, issues, and alternatives," J. Organ. Behav., pp. 667-683, 1997.

[148] H. Abdi, L. J. Williams, and D. Valentin, "Multiple factor analysis: principal component analysis for multitable and multiblock data sets," Wiley Interdiscip. Rev. Comput. Stat., vol. 5, no. 2, pp. 149-179, 2013.

[149] L. Liang, K. Hayashi, and K.-H. Yuan, "The Goodness of Sample Loadings of Principal Component Analysis in Approximating to Factor Loadings with High Dimensional Data," in Quantitative Psychology Research, Springer, 2016, pp. 199-211. 
[150] J. M. Conway and A. I. Huffcutt, "A review and evaluation of exploratory factor analysis practices in organizational research," Organ. Res. methods, vol. 6, no. 2, pp. 147-168, 2003.

[151] S. Wold, K. Esbensen, and P. Geladi, "Principal component analysis," Chemom. Intell. Lab. Syst., vol. 2, no. 1-3, pp. 37-52, 1987.

[152] M. Basto, J. M. Pereira, and others, “An SPSS R-menu for ordinal factor analysis," J. Stat. Softw., vol. 46, no. 4, pp. 1-29, 2012.

[153] C. Stergiou and D. Siganos, "Neural Networks. 1996.” 2010.

[154] L. Bottou, "Large-scale machine learning with stochastic gradient descent," in Proceedings of COMPSTAT'2010, Springer, 2010, pp. 177-186.

[155] B. A. Cerny and H. F. Kaiser, "A study of a measure of sampling adequacy for factoranalytic correlation matrices," Multivariate Behav. Res., vol. 12, no. 1, pp. 43-47, 1977.

[156] W. R. Zwick and W. F. Velicer, "Factors influencing four rules for determining the number of components to retain," Multivariate Behav. Res., vol. 17, no. 2, pp. 253-269, 1982.

[157] H. F. Kaiser, “A second generation little jiffy,” Psychometrika, vol. 35, no. 4, pp. 401-415, 1970.

[158] B. Williams, A. Onsman, and T. Brown, "Exploratory factor analysis: A five-step guide for novices," Australas. J. Paramed., vol. 8, no. 3, 2010.

[159] C. D. Dziuban and E. C. Shirkey, "When is a correlation matrix appropriate for factor analysis? Some decision rules.," Psychol. Bull., vol. 81, no. 6, p. 358, 1974.

[160] H. Zou, T. Hastie, and R. Tibshirani, "Sparse principal component analysis," J. Comput. Graph. Stat., vol. 15, no. 2, pp. 265-286, 2006.

[161] R. Bro and A. K. Smilde, "Principal component analysis," Anal. Methods, vol. 6, no. 9, pp. 2812-2831, 2014.

[162] M. W. Watkins, "Monte Carlo PCA for parallel analysis [computer software]," State Coll. PA Ed Psych Assoc., pp. 432-442, 2000.

[163] M. W. Watkins, "Determining parallel analysis criteria," J. Mod. Appl. Stat. methods, vol. 
5 , no. 2, p. 8, 2005.

[164] E. R. Girden and R. Kabacoff, Evaluating research articles from start to finish. Sage, 2010.

[165] A. Candel, V. Parmar, E. LeDell, and A. Arora, “Deep learning with H2O,” H2O. ai Inc, 2016.

[166] M. Sokolova, N. Japkowicz, and S. Szpakowicz, "Beyond accuracy, F-score and ROC: a family of discriminant measures for performance evaluation," in Australasian joint conference on artificial intelligence, 2006, pp. 1015-1021.

[167] B. Lütkenhöner and T. Basel, "Predictive modeling for diagnostic tests with high specificity, but low sensitivity: a study of the glycerol test in patients with suspected Menieres disease," PLoS One, vol. 8, no. 11, p. e79315, 2013.

[168] D. J. Ketchen Jr and C. L. Shook, "The application of cluster analysis in strategic management research: an analysis and critique," Strateg. Manag. J., pp. 441-458, 1996.

[169] L. T. Dunn, "RAISED INTRACRANIAL PRESSURE," J. Neurol. Neurosurg. Psychiatry, vol. 73, no. suppl 1, pp. i23--i27, 2002.

[170] T. B. Ducker, R. L. Simmons, and R. W. Anderson, "Increased Intracranial Pressure and Pulmonary Edema: Part 3: The Effect of Increased Intracranial Pressure on the Cardiovascular Hemodynamics of Chimpanzees," J. Neurosurg., vol. 29, no. 5, pp. 475483, 1968.

[171] C. S. Ogilvy and A. B. DuBois, "Effect of increased intracranial pressure on blood pressure, heart rate, respiration and catecholamine levels in neonatal and adult rabbits," Neonatology, vol. 52, no. 6, pp. 327-336, 1987. 